EUROPEAN ORGANIZATION FOR NUCLEAR RESEARCH

CERN-PPE/96-89

CMU-HEP/96-04

27 June 1996

\title{
Luminosity Measurement in the L3 Detector at LEP
}

I.C. Brock, A. Engler, T. Ferguson, F. Filthaut, R.W. Kraemer, M. Merk, C. Rippich, X. Shi, J. Shukla, R.B. Sutton, G. Tsipolitis, H. Vogel, J. You, P. Lecoq, G.J. Bobbink, ${ }^{1,3}$ J. Buskens, I. Cerjak, H. Groenstege, E. Koffeman, F.L. Linde, ${ }^{3}, 2,3$ G. Raven, P. Rewiersma, H.W.A. Schuijlenberg, A. de Waard, V. Commichau, K. Hangarter, P. Schmitz.

1 Carnegie Mellon University, Pittsburgh, PA 15213, USA

2 CERN, 1211 Genève 23, Switzerland

3 National Institute for High Energy Physics, NIKHEF, 1009 DB Amsterdam, The Netherlands

4 III. Physikalisches Institut, RWTH, 52056 Aachen, Germany

\begin{abstract}
One of the limiting factors in the determination of the electroweak parameters from cross section measurements of $\mathrm{e}^{+} \mathrm{e}^{-}$annihilation close to the $\mathrm{Z}$ pole is the precision of the luminosity measurement. The luminosity monitor of the L3 detector at LEP and the analysis of its data are described. Using a combination of a BGO calorimeter and a 3-layer silicon tracker, the absolute luminosity has been measured with an experimental precision of $0.08 \%$ in 1993 and $0.05 \%$ in 1994 . The measurement relies on a detailed understanding of small-angle elastic $\mathrm{e}^{+} \mathrm{e}^{-}$ (Bhabha) scattering from the experimental and theoretical point of view, as well as an excellent knowledge of the detector geometry.
\end{abstract}

Submitted to Nuclear Instruments and Methods A 


\section{Introduction}

The integrated luminosity serves as an absolute normalization of event rates observed in detectors. In $\mathrm{e}^{+} \mathrm{e}^{-}$colliders Bhabha scattering at small angles, $\mathrm{e}^{+} \mathbf{e}^{-} \rightarrow \mathrm{e}^{+} \mathbf{e}^{-}(\gamma)$, is usually used to measure the luminosity. Its cross section can be calculated with high precision from Quantum Electrodynamics and depends only weakly on the properties of the Z, even when running at center-of-mass energies close to the $\mathrm{Z}$ pole.

To lowest order, the small-angle Bhabha cross section (integrated over the azimuthal angle, $\phi)$, in a detector with a polar angle coverage from $\theta_{\min }$ to $\theta_{\max }$, is given by:

$$
\sigma=\frac{16 \pi \alpha^{2}}{s}\left(\frac{1}{\theta_{\min }^{2}}-\frac{1}{\theta_{\max }^{2}}\right)
$$

where $\alpha$ is the fine-structure constant and $s$ is the square of the center-of-mass energy.

To determine the luminosity accurately, the most important features of a detector are:

- well-known geometry;

- very high and well-known trigger efficiency;

- small and well-understood backgrounds;

- full coverage of the azimuthal angle.

The last item ensures that small transverse offsets of the detector with respect to the beam have a negligible effect on the luminosity determination.

These requirements are realized in the L3 experiment at LEP with a luminosity monitor consisting of a calorimeter made of Bismuth Germanate (BGO) crystals, which provides excellent energy resolution and a very efficient trigger, complemented by a tracker made of single-sided silicon wafers (SLUM). The wafers have very high intrinsic geometrical precision $(1-2 \mu \mathrm{m})$ and can be accurately positioned and measured $(6 \mu \mathrm{m})$. An overview of the position of the detector in the L3 experiment is shown in Figure 1. The luminosity detector is situated at small polar angles, so that the Bhabha cross section is larger than that for hadron production and the interference between the photon and $\mathrm{Z}$ exchange diagrams is small. Bhabha events are selected using the calorimetric measurement in the BGO to provide a background free sample of events, and the silicon tracker to select only those Bhabhas that are contained in a precisely defined fiducial volume.

The L3 coordinate system has the $x$-axis pointing to the center of the LEP ring, the $y$ axis pointing vertically upwards and the $z$-axis in the direction of the electron beam. In this coordinate frame the polar and azimuthal angles will be denoted as $\theta$ and $\phi$. Throughout this paper a distinction is made between the coordinates in this physics reference frame and coordinates in the local luminosity detector reference frame $r$ and $\varphi$.

At the time of the original design of the L3 luminosity monitor a goal of a luminosity measurement with $1 \%$ precision was set [1]. Using the BGO calorimeter alone, an accuracy of $0.6 \%$ has been achieved. However, when running at or close to the $\mathrm{Z}$ peak, the efficiency of the L3 detector for the process $\mathrm{Z} \rightarrow$ hadrons can be measured with a systematic error of about $0.1 \%$. The theoretical Bhabha cross section has also been recalculated with improved accuracy, so a measurement of the luminosity with an accuracy of about $0.1 \%$ is desirable. With the addition of the silicon tracker, the error on the luminosity measurement is very similar to that on the efficiency for detecting hadronic Z decays. 


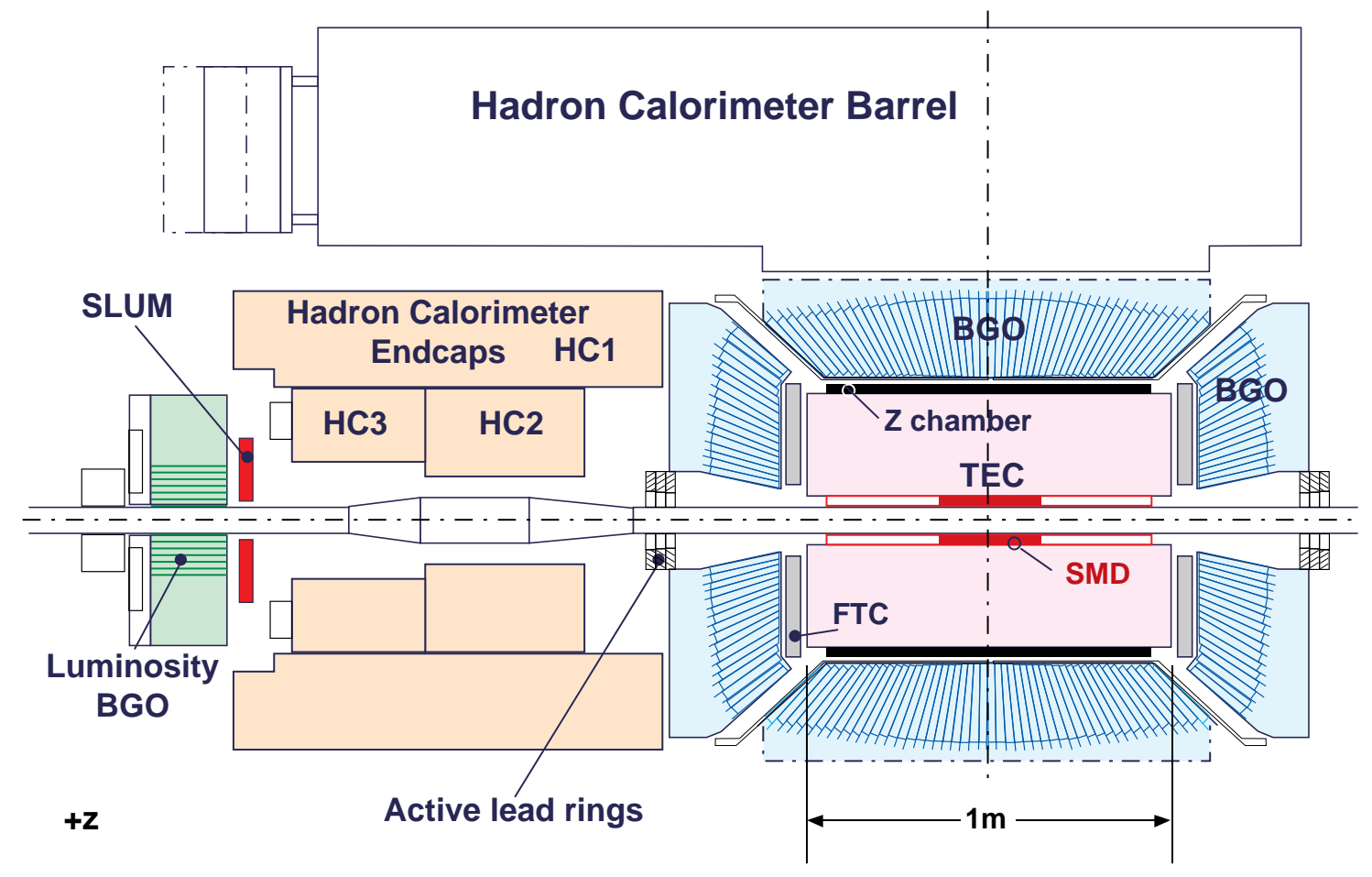

Figure 1: Side-view of the central region of the L3 experiment on the $+z$ side, showing the beam pipe and the position of the luminosity BGO calorimeter and the silicon tracker (SLUM).

The determination of the theoretical cross section for the detector uses $\mathrm{e}^{+} \mathbf{e}^{-} \rightarrow \mathbf{e}^{+} \mathbf{e}^{-}(\gamma)$ Monte Carlo events generated at $\sqrt{s}=91.25 \mathrm{GeV}$ using BHLUMI V2.01 [2] and BHLUMI V4.03 [3]. The events generated using BHLUMI V2.01 have been passed through the L3 simulation program [4].

This paper describes the luminosity monitor's status and the analysis of the 1993 and 1994 data, amounting to a total of $33+50 \mathrm{pb}^{-1}$ collected by the L3 experiment. In the next two sections of the paper the detector components and their performance are described. In the fourth section the determination of the alignment of the calorimeter and the tracker is discussed. The extraction of the integrated luminosity is described in Section 5. 


\section{The BGO Calorimeter}

The BGO calorimeter consists of two detectors, which are situated on each side of the interaction point (I.P.), at a distance of about $2730 \mathrm{~mm}$. Each calorimeter is cylindrically symmetric and consists of 304 BGO crystals parallel to the beam axis, each covering $15 \mathrm{~mm}$ radially and arranged in eight rings. Azimuthally the calorimeters are divided into 16 sectors, with each sector covering an angle of $22.4^{\circ}$. A schematic overview of the detector is shown in Figure 2.
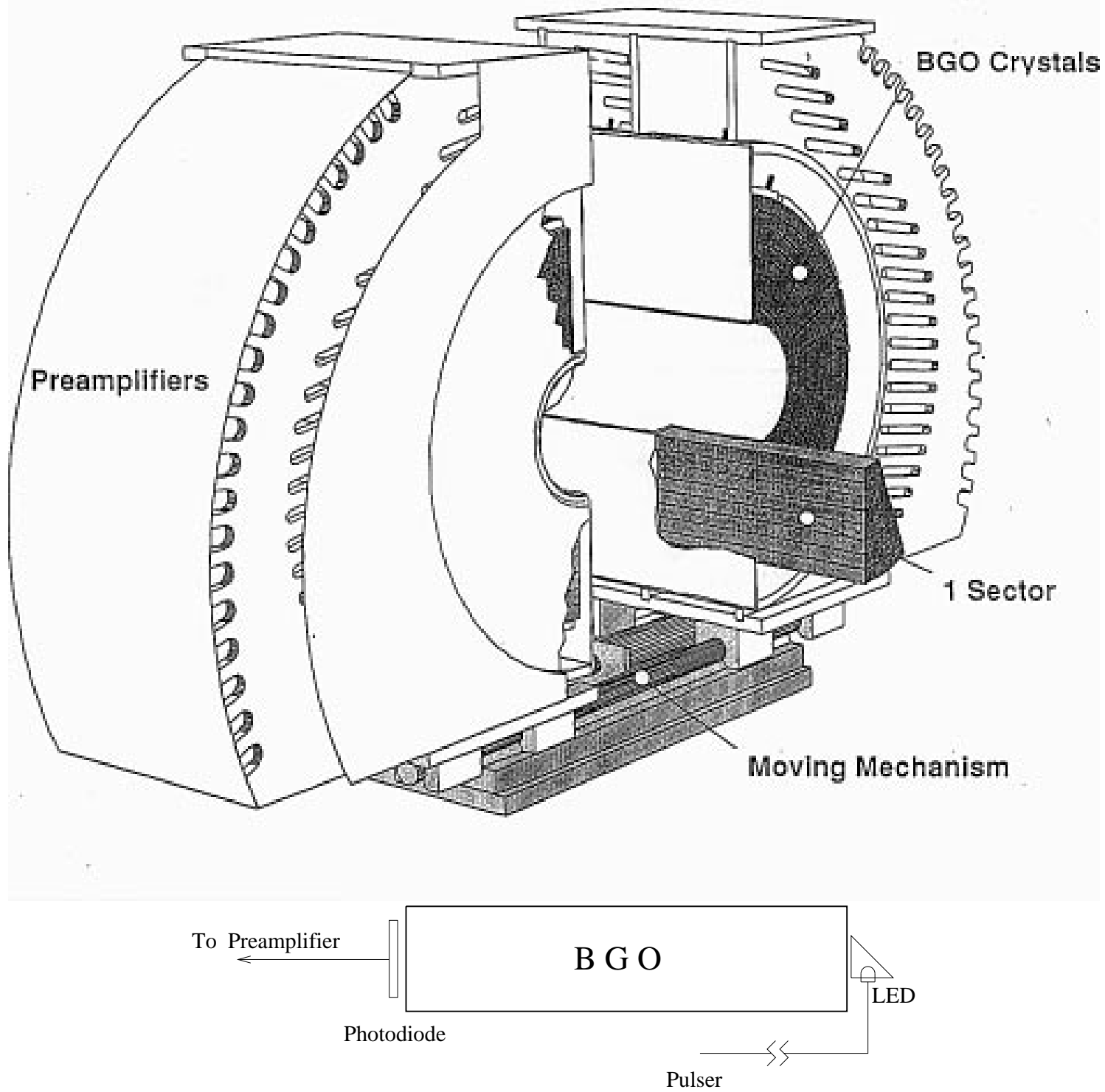

Figure 2: Overview of the BGO calorimeter showing the location of the crystals and preamplifiers and the moving mechanism. The locations of the photodiode and the LED on a crystal are also indicated.

One sector contains 19 crystals, each of which is 24 radiation lengths long with a cross section which ranges from $15 \times 15 \mathrm{~mm}^{2}$ to $15 \times 30 \mathrm{~mm}^{2}$. Each crystal is wrapped first in Teflon tape $(75 \mu \mathrm{m})$ and then in $25 \mu \mathrm{m}$ thick copper foil. Crystals from three different suppliers are used 
in the calorimeter: Nihon Kessho Koogaku, Japan (NKK); the Shanghai Institute of Ceramics, China (SIC); and Crismatec, France. Their locations are shown in Figure 3. The SIC crystals are doped with Eu whereas the NKK and the Crismatec crystals are not doped. Each crystal is read out by a Hamamatsu photodiode which is mounted on the back of the crystal. On the front side a green light emitting diode (LED) is mounted and is used for monitoring purposes. The general characteristics of the detector are summarized in Table 1.

\begin{tabular}{|lr|}
\hline Distance of the front from the I.P. & $2730 \mathrm{~mm}$ \\
Minimum radius & $68 \mathrm{~mm}$ \\
Maximum radius & $192 \mathrm{~mm}$ \\
Crystal length & $260 \mathrm{~mm}$ \\
Crystal length in radiation lengths & 24 \\
Number of crystals per sector & 19 \\
Angular coverage of a sector & $22.4^{\circ}$ \\
Number of sectors per side & 16 \\
\hline
\end{tabular}

Table 1: The general characteristics of the luminosity BGO calorimeter.

The BGO calorimeter is split into two halves (see Figure 2) which are separated during each filling of the LEP ring, in order to protect the crystals from radiation damage. The movement is controlled remotely by a hydraulic device, with a position reproducibility of better than $10 \mu \mathrm{m}$. Additional protection comes from an $8 \mathrm{~cm}$ thick lead shield behind the BGO calorimeter.

\section{$2.1 \quad$ Readout}

The L3 barrel, endcap and luminosity monitor BGO calorimeters operate in a $0.5 \mathrm{~T}$ magnetic field. Therefore, photodiodes (Hamamatsu S1790 (S2662 for the ring 1, 5 and 6 crystals)) are used to detect the BGO scintillation light. The signal from the photodiode typically corresponds to about 1200 electrons for each $\mathrm{MeV}$ of deposited energy.

All the BGO calorimeters in the L3 experiment have almost identical front-end electronics and follow the same readout chain. The readout sequence is shown in Figure 4. A more detailed schematic of the front-end electronics is shown in Figure 5. The signal from the photodiode is passed to a charge-sensitive preamplifier [5] mounted $30 \mathrm{~cm}$ away from the crystal, which uses a low noise, high transconductance Toshiba 2SK147 FET in a cascode configuration. The output pulse rise time is $300 \mathrm{~ns}$ (corresponding to the BGO light decay time) and the exponential decay time is $200 \mu \mathrm{s}$. The signal is then fed to the analog to digital converter (ADC), one for each crystal, which is mounted a further $3 \mathrm{~m}$ away. The signal from the preamplifier is differentiated with a pole-zero circuit which replaces the long decay time with one of $1.1 \mu \mathrm{s}$. More details about the ADC boards and their characteristics can be found in Reference [6]. Once the signals are digitized by the ADC boards they follow the same logic as the signals from the barrel and endcap BGO crystals, which is also described in Reference [6].

Figure 6 shows the time evolution of the pedestal and its r.m.s. width for a single crystal during the 1993 running period. For most of the crystals the pedestals are between 70 and $90 \mathrm{MeV}$ and the pedestal widths are between 1 and $3 \mathrm{MeV}$. During the running period of one year there are usually $2-3$ dead or malfunctioning crystals. 

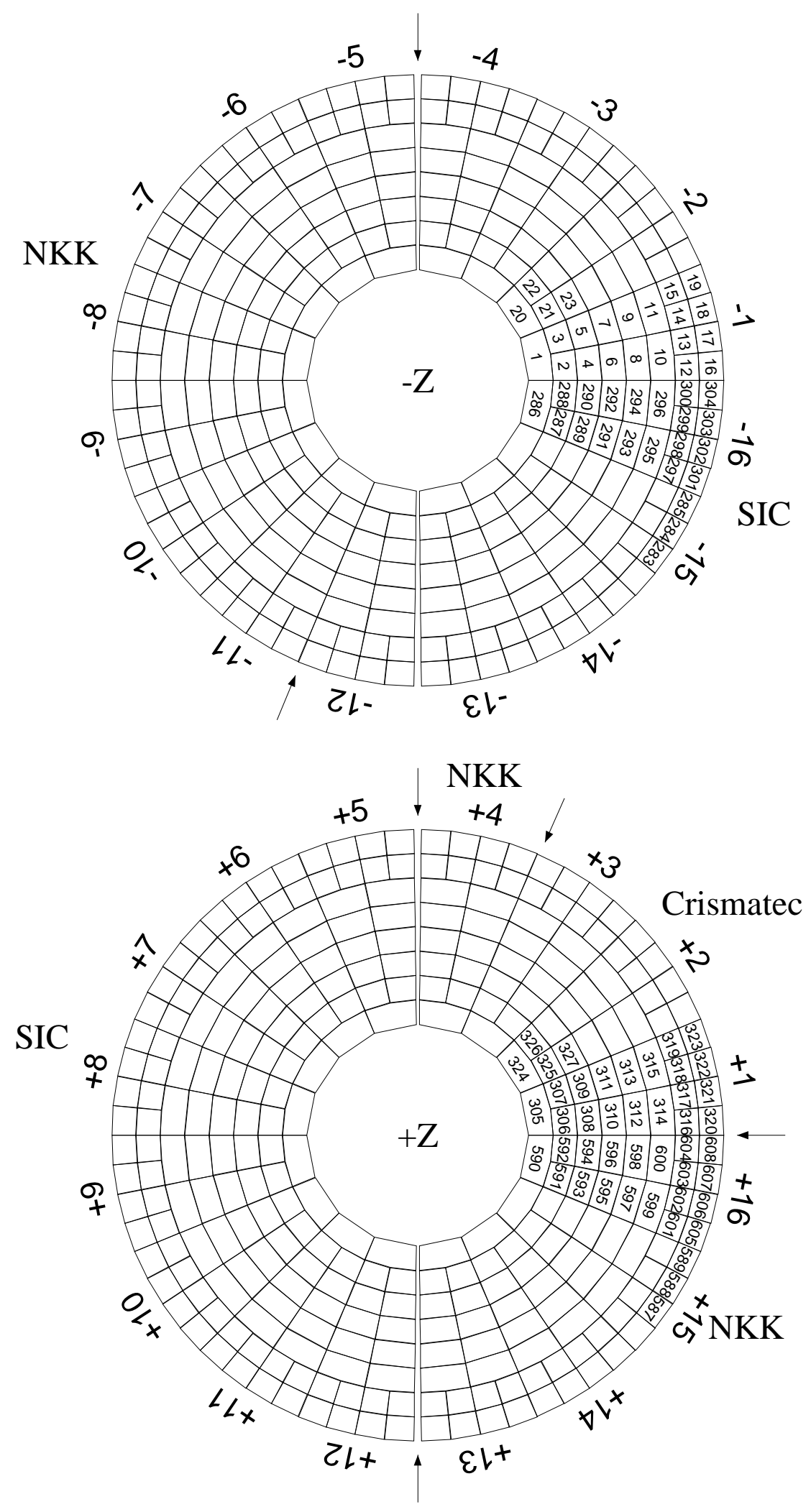

Figure 3: The layout of the BGO crystals in the luminosity calorimeter. The numbering of the sectors and the number assigned to each crystal are indicated. The locations of the crystals from the different manufacturers are also shown. 


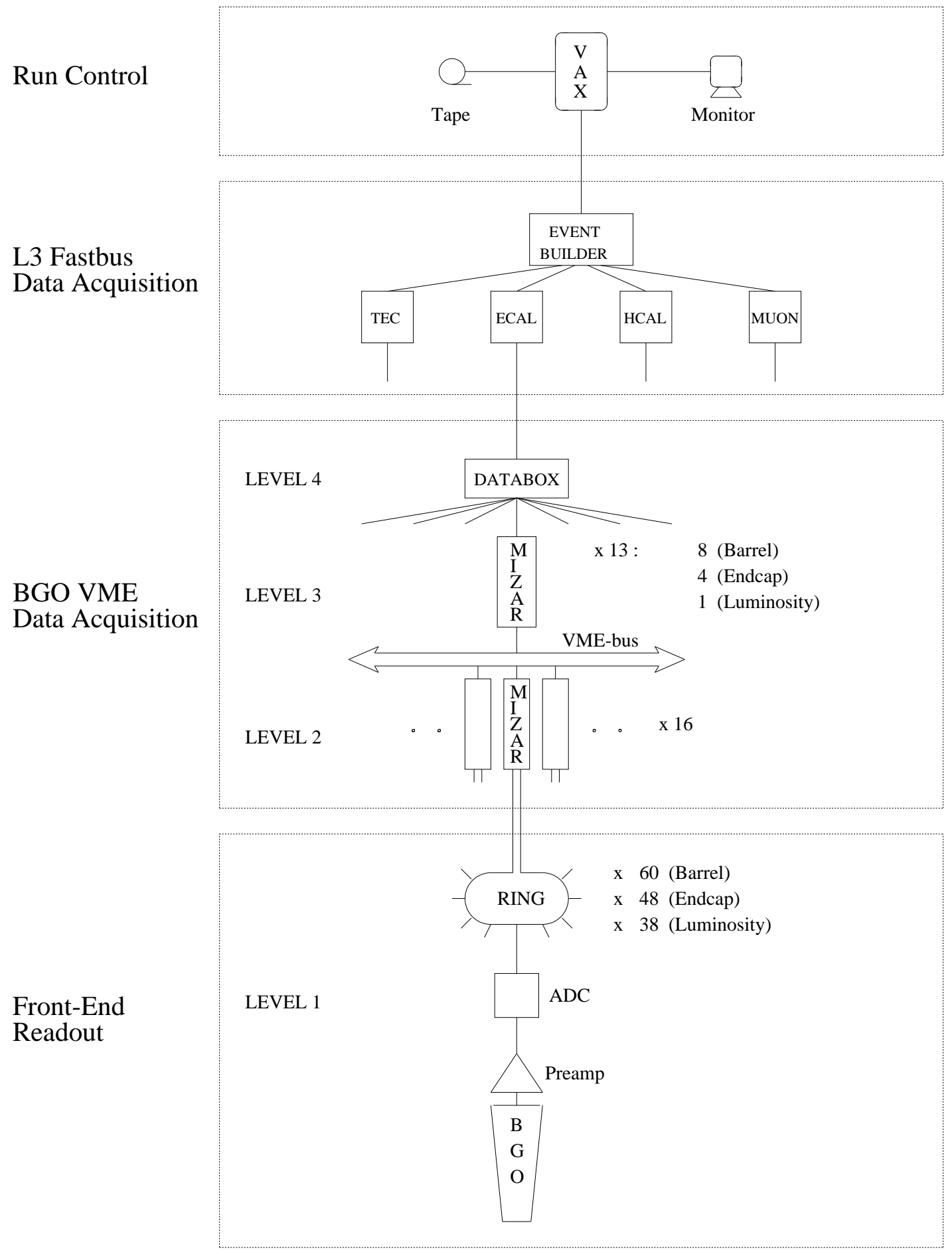

Figure 4: The layout of the BGO readout. After the front-end electronics described in the text, the data are collected in a VME system and then transferred to the main L3 Fastbus readout chain. 


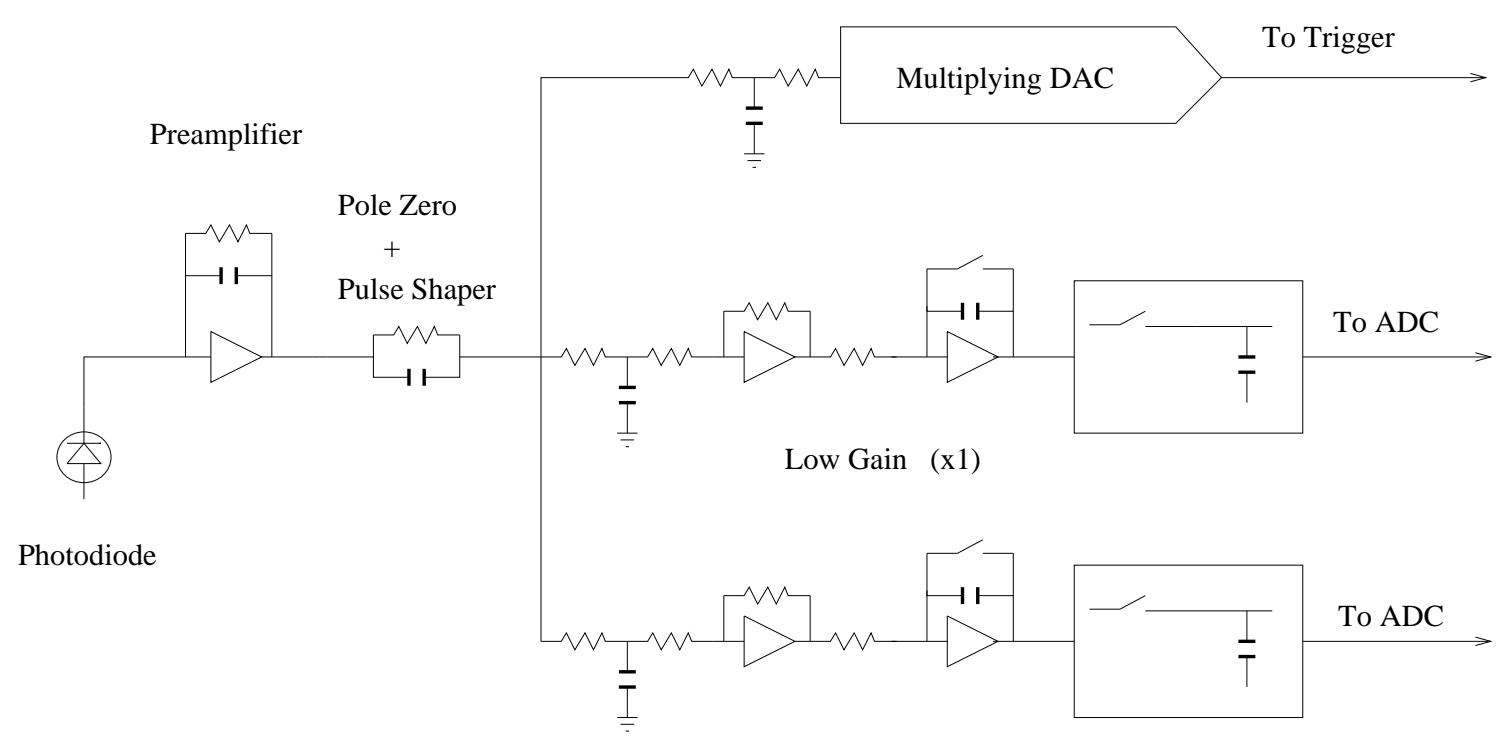

High Gain (x32)

Figure 5: The layout of the front-end electronics.

\subsection{Trigger}

The analog trigger signals from each crystal are summed into sectors on an "analog sum" card located next to the ADC boards. These cards (one for each detector) then drive the signal for each sector differentially through a $40 \mathrm{~m}$ twisted pair cable to a CAMAC receiver card. The signals are then digitized using two 16-channel Lecroy Fast Encode and Readout ADCs (FERA 4300B) which have a sensitivity of about $300 \mathrm{MeV} / \mathrm{ADC}$ count. Three different triggers are constructed using these digital energies: The "luminosity" trigger requires backto-back segments with an energy exceeding a threshold of $15 \mathrm{GeV}$ on each side. To avoid edge effects the energies of each pair of adjacent sectors are summed together. For the "double-tag" trigger it is required that the total energy in the calorimeters be more than $25 \mathrm{GeV}$ for the larger measured energy and $5 \mathrm{GeV}$ for the smaller, irrespective of their angular correlation. To monitor the trigger efficiency, a "single-tag" trigger which requires an energy larger than $30 \mathrm{GeV}$ deposited in at least one of the two calorimeters is used. This single-tag trigger is prescaled by a factor of 40. Details on the hardware of the first-level trigger used in the luminosity monitor can be found in Reference [7]. The detector is read out if any of the trigger requirements is satisfied.

The trigger decision is ready about $1 \mu$ s before the next bunch crossing. At a luminosity of $10^{31} \mathrm{~cm}^{-2} \mathrm{~s}^{-1}$, the rate of the luminosity and the double-tag triggers is of the order of $2-3 \mathrm{~Hz}$, while the rate for the prescaled single-tag trigger is about $0.2 \mathrm{~Hz}$.

The events that pass all the Bhabha selection criteria, but were triggered by the singletag trigger only, indicate a trigger inefficiency. Assuming that the inefficiency is small and uncorrelated between the two detectors, the trigger inefficiency, $(1-\varepsilon)$, is:

$$
(1-\varepsilon)=N_{\text {Bhabha }}^{s t} / N_{\text {Bhabha }}^{s t+d t}
$$

where $N_{\text {Bhabha }}^{s t}$ is the number of selected Bhabhas that fired the single-tag trigger only, while $N_{\text {Bhabha }}^{s t+d t}$ is the number of selected Bhabhas that fired either the single-tag or both the single-tag and the double-tag triggers. 
Crystal 134

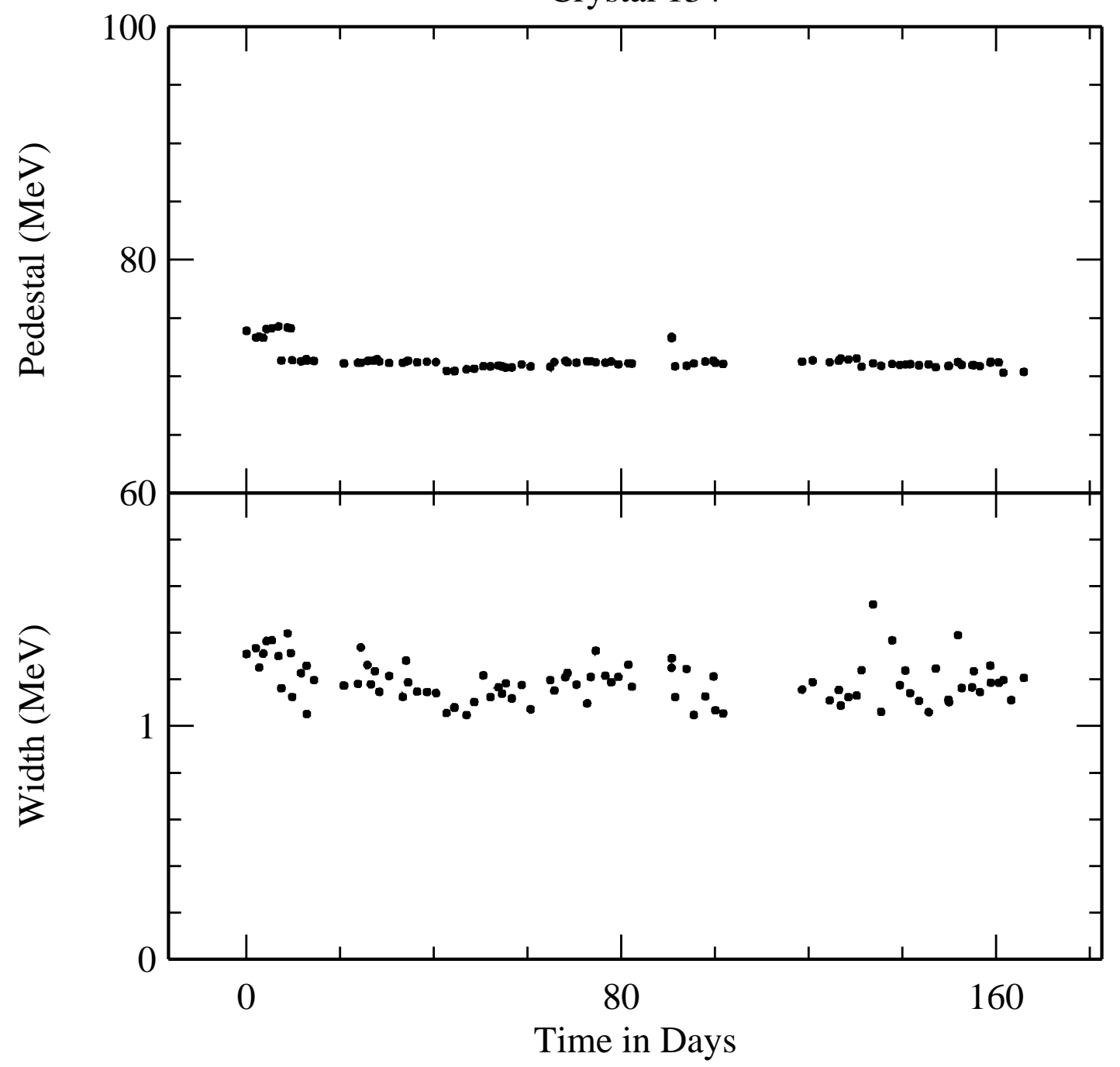

Figure 6: The average pedestal and pedestal width of a typical crystal during the 1993 running period. 
There are no events that fail the double-tag trigger out of a sample of 187000 single-tag triggers that pass the Bhabha selection criteria in 1993 and 1994 . Due to its symmetric energy cut and back-to-back requirement the luminosity trigger has a significant inefficiency $(0.1-0.5 \%$ depending on the cuts). The double-tag trigger is more sensitive to machine backgrounds, but the LEP background rates have been low enough that it has always been used.

\subsection{Position Reconstruction and Energy Determination Algorithm}

For electromagnetic showers, the $r, \varphi$ impact coordinates on the BGO calorimeter, as well as the energy, $E$, of the incident electron, positron or photon, are determined from the observed energies in the crystals. To handle different crystal shapes, cracks between crystals and to deal with edge effects, as well as malfunctioning or dead crystals, an algorithm based on the average transverse shape of electromagnetic showers as predicted by GEANT is used.

This transverse shape is used to parameterize the energy distribution of an electromagnetic shower with respect to its center: ${ }^{1)}$

$$
\mathcal{S}(\rho)=A \times \delta_{\rho=0}+\sum_{i=1}^{2} \frac{A_{i}}{\sigma_{i}} \times e^{-\rho / \sigma_{i}} \quad \text { with } \quad \int_{0}^{\infty} S(\rho) \rho d \rho=\frac{1}{2 \pi},
$$

i.e. the energy fraction deposited in a ring of width $d \rho$ at a distance $\rho$ from the shower center is equal to $\mathcal{S}(\rho) \rho d \rho$. This parameterization and the symmetry of the BGO calorimeter layout are used to create a database entry which makes it possible to obtain directly, for given impact coordinates $r, \varphi$ of a particle, the average fractional energy, $\mathcal{E}_{i}^{r, \varphi}$, in crystal number $i$. Typical uncertainties in the impact coordinates are $\mathcal{O}(1 \mathrm{~mm})$. The changes in $\mathcal{E}_{i}^{r, \varphi}$ under $\mathcal{O}(1 \mathrm{~mm})$ changes of these coordinates contribute to the uncertainty, $\Delta \mathcal{E}_{i}^{r, \varphi}$, on the average fractional energy in crystal number $i$. This contribution is only significant, $\geq 10 \%$, for impact coordinates near the edge of a crystal. Other contributions to the fractional energy uncertainty are detector noise, a few $\mathrm{MeV}$ on the energy measured by a crystal, and shower fluctuations. These effects are only important for the crystals which are at least a few $\mathrm{cm}$ away from the impact coordinate.

A $\chi^{2}$ minimization procedure is used to determine the optimal $r, \varphi$ impact coordinates to be assigned to an electromagnetic cluster defined by an arbitrary collection of $n$ crystals with recorded energies $E_{i}$. To compare the observed crystal energies to the expected energy distribution, each crystal energy is normalized by the total energy observed in the cluster ( $\left.E_{\text {observed }}\right)$ :

$$
E_{i} \rightarrow e_{i}=\frac{E_{i}}{E_{\text {observed }}} \quad \text { with } \quad E_{\text {observed }} \equiv \sum_{i=1}^{n} E_{i}
$$

where $i=1,2, \ldots n$ runs over the number of crystals in the cluster. For a typical $45 \mathrm{GeV}$ cluster and a cut on the energy deposited in a single crystal of $200 \mathrm{MeV}, n \approx 12$. Similarly, the predicted energy distribution must be normalized by the total fraction of the energy predicted in the cluster $\left(\mathcal{E}_{\text {pred }}^{r, \varphi}\right)$ :

$$
\mathcal{E}_{i}^{r, \varphi} \rightarrow \epsilon_{i}^{r, \varphi}=\frac{\mathcal{E}_{i}^{r, \varphi}}{\mathcal{E}_{\text {pred }}^{r, \varphi}}, \quad \Delta \mathcal{E}_{i}^{r, \varphi} \rightarrow \Delta \epsilon_{i}^{r, \varphi}=\frac{\Delta \mathcal{E}_{i}^{r, \varphi}}{\mathcal{E}_{\text {pred }}^{r, \varphi}} \quad \text { with } \quad \mathcal{E}_{\text {pred }}^{r, \varphi} \equiv \sum_{i=1}^{n} \mathcal{E}_{i}^{r, \varphi} \quad \text { and } \quad \sum_{i=1}^{n} \epsilon_{i}^{r, \varphi}=1
$$

\footnotetext{
${ }^{1)}$ The actual parameters used are: $A=0.10, A_{1}=0.48, A_{2}=0.42$ and $\sigma_{1}=2.3 \mathrm{~mm}, \sigma_{2}=12.7 \mathrm{~mm}$. The exact form of the parameterization is irrelevant provided it has cylindrical symmetry and resembles real shower profiles. Ideally the average of many simulated transverse shower shapes could be used directly for the creation of the database entry.
} 
$E_{\text {observed }}$ has dimension $[\mathrm{GeV}]$, while $\mathcal{E}_{\text {pred }}^{r, \varphi}$ is dimensionless and normally close to unity. An $\mathcal{E}_{\text {pred }}^{r, \varphi}$ which differs from unity indicates a cluster with large missing energy, either due to edge effects or to the presence of a dead crystal. The $\chi^{2}$ to be minimized is defined as:

$$
\chi^{2}=\sum_{i=1}^{n}\left(\frac{e_{i}-\epsilon_{i}^{r, \varphi}}{\Delta \epsilon_{i}^{r, \varphi}}\right)^{2}
$$

The minimization is performed in an iterative way with respect to the impact coordinates $r, \varphi$ of the particle. The procedure starts with the $r, \varphi$ given by the "center-of-gravity" method and subsequently calculates the $\chi^{2}$ at this point and four surrounding points; two points each with either the radial or the azimuthal coordinate changed by equal amounts up and down. The $\chi^{2}$ values on this grid are used to improve the $r, \varphi$ impact coordinate estimate. The step sizes in $r$ and $\varphi$ start out large, typically $1 \mathrm{~mm}$ in each direction, becoming smaller each time the gradient of the $\chi^{2}$ changes direction. The convergence of the fit is based on two criteria: either both step sizes correspond to transverse spatial changes of less than $1 / 25 \mathrm{~mm}$ or the local derivative of the $\chi^{2}$ in space becomes less than $0.2 / \mathrm{mm}$. In addition, no more than 100 iterations are allowed. Typically the fit converges after 5-10 iterations. In addition to the optimal impact coordinates, this fit procedure also yields the uncertainties on the impact coordinates as well as a measure, the $\chi^{2} / D F$, of the electromagnetic origin of the fitted cluster.

Once the optimal impact coordinates $r, \varphi$ are obtained, the incident energy, $E$, of the particle is simply given by:

$$
E=\frac{E_{\text {observed }}}{\mathcal{E}_{\text {pred }}^{r, \varphi}} \equiv \frac{\sum_{i=1}^{n} E_{i}}{\sum_{i=1}^{n} \mathcal{E}_{i}^{r, \varphi}} \geq E_{\text {observed }} \quad \text { since } \quad \mathcal{E}_{\text {pred }}^{r, \varphi} \leq 1
$$

The power of this algorithm is demonstrated in Figure 7. In this figure two electromagnetic showers are fitted twice: once with all crystals functional and once with the central crystal declared dead. The fitted impact coordinates for the two cases are in excellent agreement. Taking into account the fact that in the second case about half of the energy is missing, the fitted energies are in reasonable agreement. For the second shower, even though its center is in ring 1 , the reconstructed energy and position are not affected much by the dead central crystal.

\section{$2.4 \quad$ Energy Calibration}

The energy calibration of the BGO crystals, i.e. the conversion from measured ADC values to deposited energy for each BGO crystal, $j$, is given by:

$$
E_{j}=\left(A D C_{j}-P E D_{j}\right) \times G A I N_{j}
$$

The pedestal for each channel $\left(P E D_{j}\right)$ is determined on a fill-to-fill basis. Pedestal drifts within a fill are very small and do not degrade the energy resolution.

The gain for each channel $\left(G A I N_{j}\right)$ is determined in an iterative way using a clean sample of Bhabha events with a tight acollinearity cut $\left(\zeta<0.25^{\circ}\right)$ and energies within $10 \%$ of the beam energy $\left(E_{\text {beam }}\right)$. Given a set of gain constants, the energy sharing between the crystals is determined. Subsequently, the mean square deviation from the beam energy is minimized:

$$
\chi^{2}=\sum_{n=1}^{N}\left(\sum_{j} A D C_{j}^{n} \times G A I N_{j}-E_{\mathrm{beam}} \times \mathcal{E}_{\text {pred }}^{r, \varphi}\right)^{2},
$$



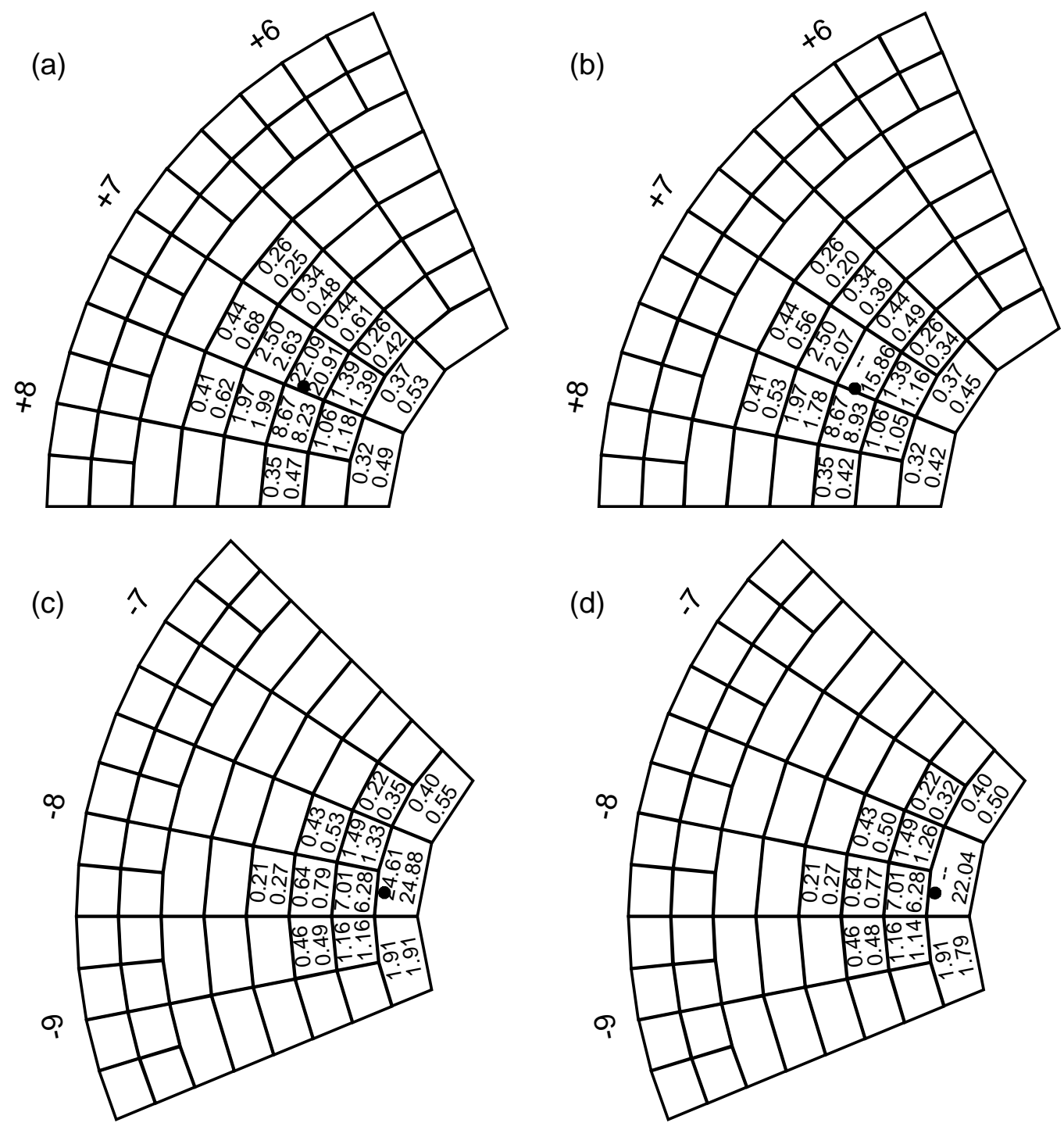

Figure 7: Two electromagnetic showers measured by the crystals of the BGO calorimeter. (a) All crystals working, $E=(45.5 \pm 0.7) \mathrm{GeV}, r=108.7 \mathrm{~mm}, \varphi=156.7^{\circ}, \chi^{2} / D F=5.5 / 12$, (b) Central crystal dead, $E=(38.6 \pm 6.5) \mathrm{GeV}, r=108.6 \mathrm{~mm}, \varphi=157.1^{\circ}, \chi^{2} / D F=5.7 / 11$, (c) All crystals working, $E=(45.4 \pm 0.7) \mathrm{GeV}, r=81.6 \mathrm{~mm}, \varphi=174.0^{\circ}, \chi^{2} / D F=2.0 / 8$, (d) Central crystal dead, $E=(42.4 \pm 1.3) \mathrm{GeV}, r=82.1 \mathrm{~mm}, \varphi=174.1^{\circ}, \chi^{2} / D F=4.8 / 7$. The measured and the fitted energies are shown in $\mathrm{GeV}$. The outermost energy in each crystal is the measured energy and the innermost is the fit energy. The reconstructed impact coordinates are shown as filled circles. 
where $N$ is the number of events in the sample used for the calibration and $\mathcal{E}_{\text {pred }}^{r, \varphi}$ is the total fraction of energy predicted in the cluster, as defined in the previous section. This procedure converges after 1-2 iterations and yields calibration constants with a statistical accuracy which varies from $0.01 \%$ for the innermost crystal ring to $0.1 \%$ for the outermost crystal ring. The calibration is performed for a continuous LEP running period, avoiding any periods affected by radiation damage to the crystals. The energy distribution for the final Bhabha sample is shown in Figure 8 . An energy resolution, $\sigma_{E}$, of about $1.3 \%$ is obtained. Although a somewhat improved resolution could probably be achieved by taking into account the temperature and gain variations with time, this value is sufficient for the luminosity measurement. From a comparison of the mean energy of the events in the data and the Monte Carlo after the calibration, the systematic error on the energy scale is estimated to be $0.1 \%$.

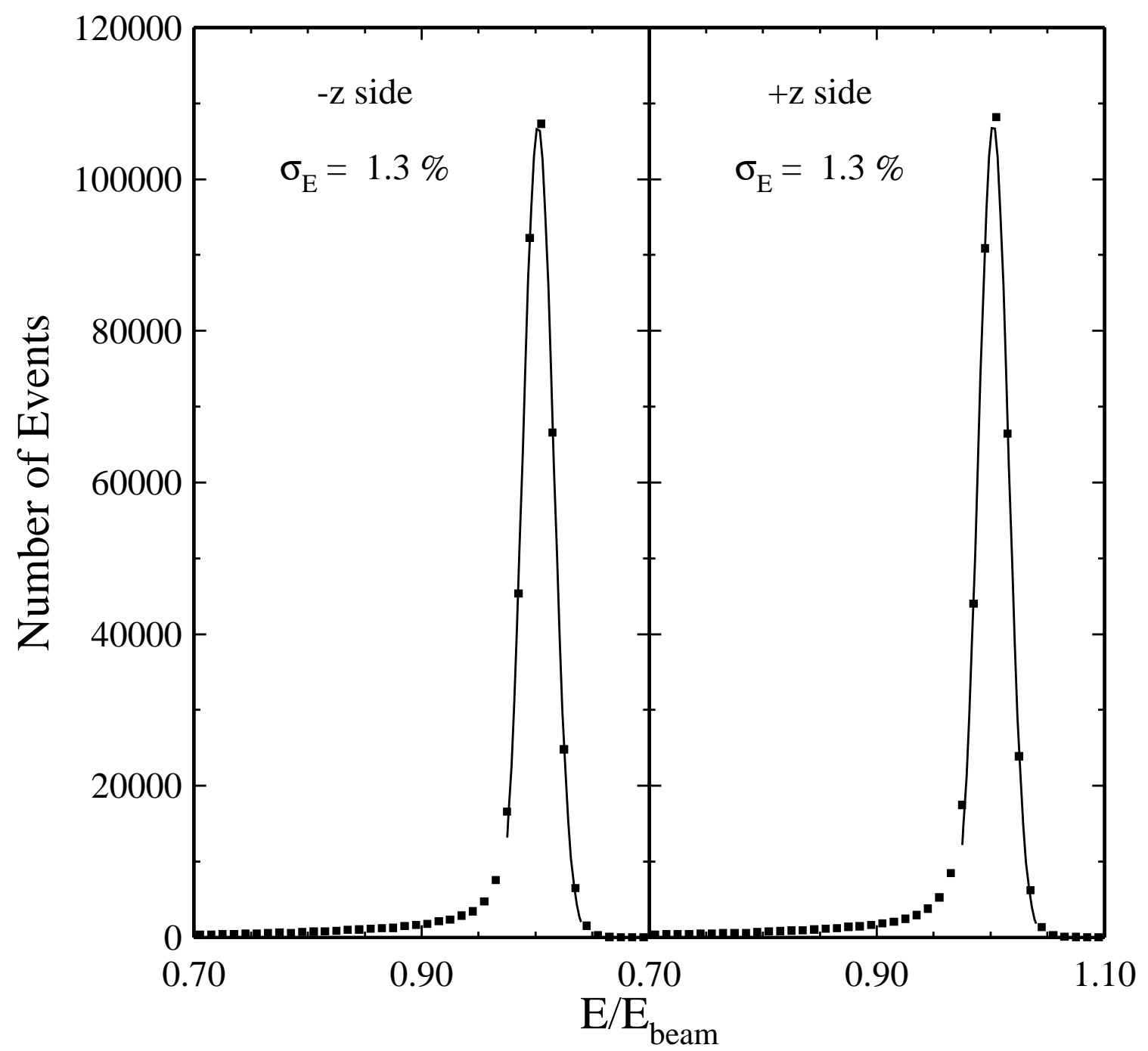

Figure 8: The BGO energy distribution for the two detectors using the 1993 Bhabha sample. A Gaussian fit to the region indicated is also shown. 


\subsection{LED System and Radiation Damage}

The performance of the BGO crystals is monitored using LEDs. For this purpose green HLMP1540 LEDs from Hewlett Packard are used. As indicated in Figure 2 an LED is mounted on the front of each BGO crystal. LED runs are taken normally once a day between LEP fills. This way a day-to-day performance of each crystal is recorded.

Incidents where the LEP beam was lost close to the L3 experiment have caused radiation damage to the crystals. This results in a change of the crystal transparency. BGO crystals are known to recover from this damage with time [8]. This effect can be monitored very well with the LED system.

The LED data in the period 1989-1993 have been studied. The data are normalized to the beginning of the running period for each year. Severe damage occurred in the runs of 1989 and 1991. The LED pulse height of the crystals was fit with a function, $f(t)$ :

$$
f(t)=a \cdot\left(1-b \cdot e^{-\left(t-t_{0}\right) / \tau}\right)
$$

where $a$ takes into account the possibility that the crystal did not fully recover to its original pulse height, $t_{0}$ is the time of the incident, $t$ is the time, $\tau$ is the recovery time and the product $a b$ gives the magnitude of the initial damage.

Crystals that showed a damage of more than $2 \%$ are fit using the above formula. The results of the fit for different crystals and different years are shown in Figure 9. Note that the NKK crystals show much longer recovery times after the 1992 incident (shown with diamonds in Figure 9). This effect is probably related to the fact that these crystals did not fully recover during the 1989 and 1991 radiation damage incidents. Figure 10 shows the percentage of the final recovery for the 1989 incident where the biggest damage to the crystals occurred.

In Reference [8] it was found that the recovery of the BGO crystals after radiation damage could be described with 3 exponentials, including a fast component of the order of 100 minutes. At LEP it is difficult to extract the fast component, since there is usually a period of a few hours after the damage before an LED run is taken. Another difference from Reference [8] is that there is no knowledge of the amount of radiation to which the crystals were exposed.

If the damage is localized in the first few centimeters of the crystal, which would be the case for damage from synchrotron radiation, one would expect that this would affect more the pulse height from the LED pulser runs than real Bhabha events. This hypothesis was checked with Bhabha events from the data. ${ }^{2}$ The agreement between the LED data and the Bhabha data is excellent (see Figure 11) and shows that the damage was not only on the crystal surface and was therefore caused by high-energy electrons and positrons from the beam and not by synchrotron radiation. Since the middle of 1992 there have been no significant radiation incidents. This is probably due to the better beam monitoring installed in the machine and the experiments, mainly to protect the experiments' silicon microvertex detectors.

\subsection{Detector Monitoring}

About $20 \%$ of the Bhabha triggers are analyzed online using the same program that is used for the standard luminosity analysis described below. This allows all aspects of the detector performance to be monitored and avoids having to maintain two sets of analysis code. In particular, the trigger efficiency (for both the BGO and the fast trigger for the silicon tracker),

\footnotetext{
2) For the Bhabha data one calibration constant for each crystal for the whole year under study was used in order to avoid possible problems due to different calibration constants for different periods in the same year.
} 


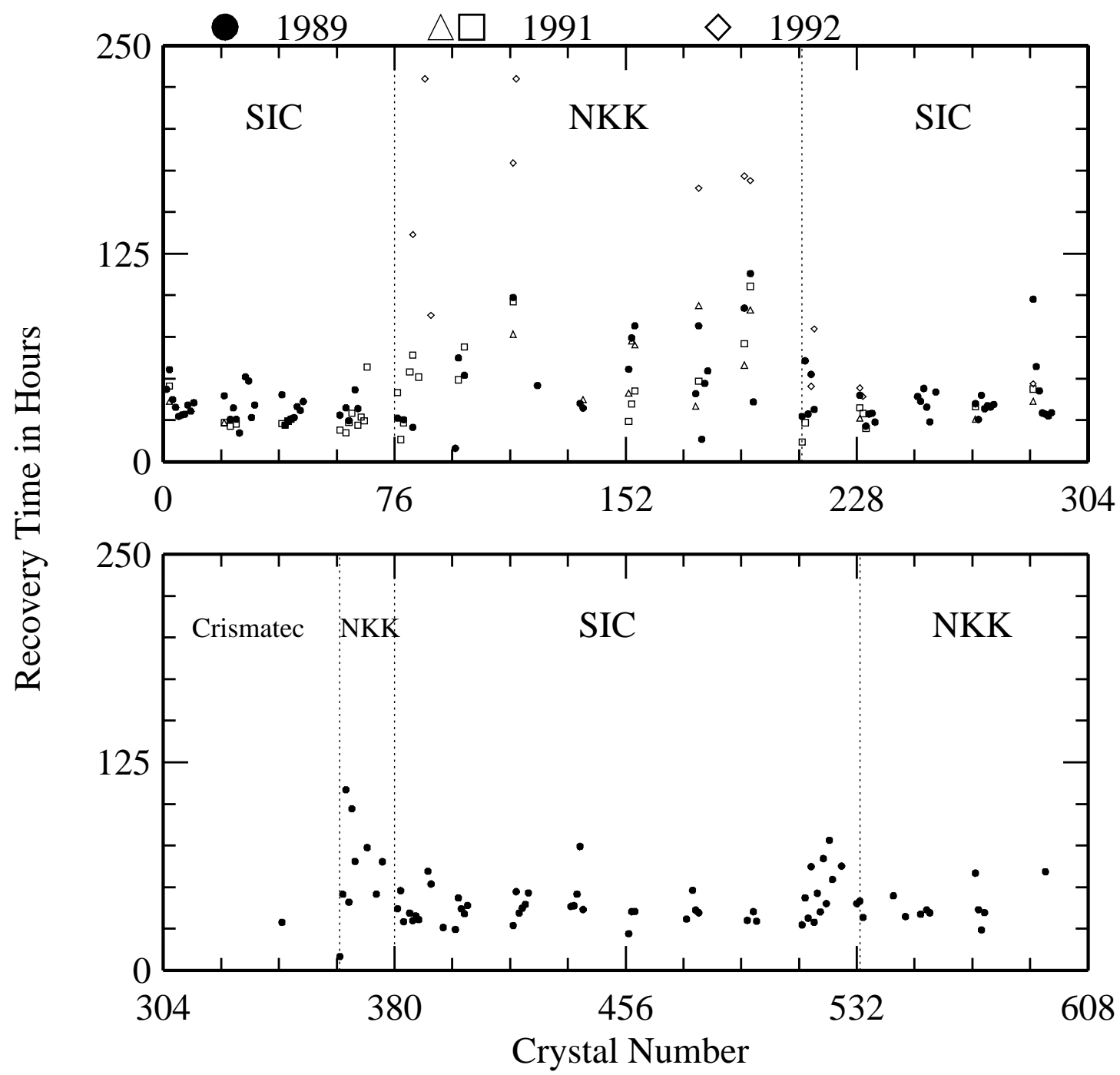

Figure 9: The recovery times for BGO crystals from different suppliers. The full dots correspond to the radiation damage during the 1989 incident, the squares and the triangles to the two May 1991 incidents and the diamonds to the 1992 incident. Note that for two crystals the recovery time has been put to 230 hours in order to be visible in the plot. The real recovery times measured are 435 and 448 hours, respectively. 


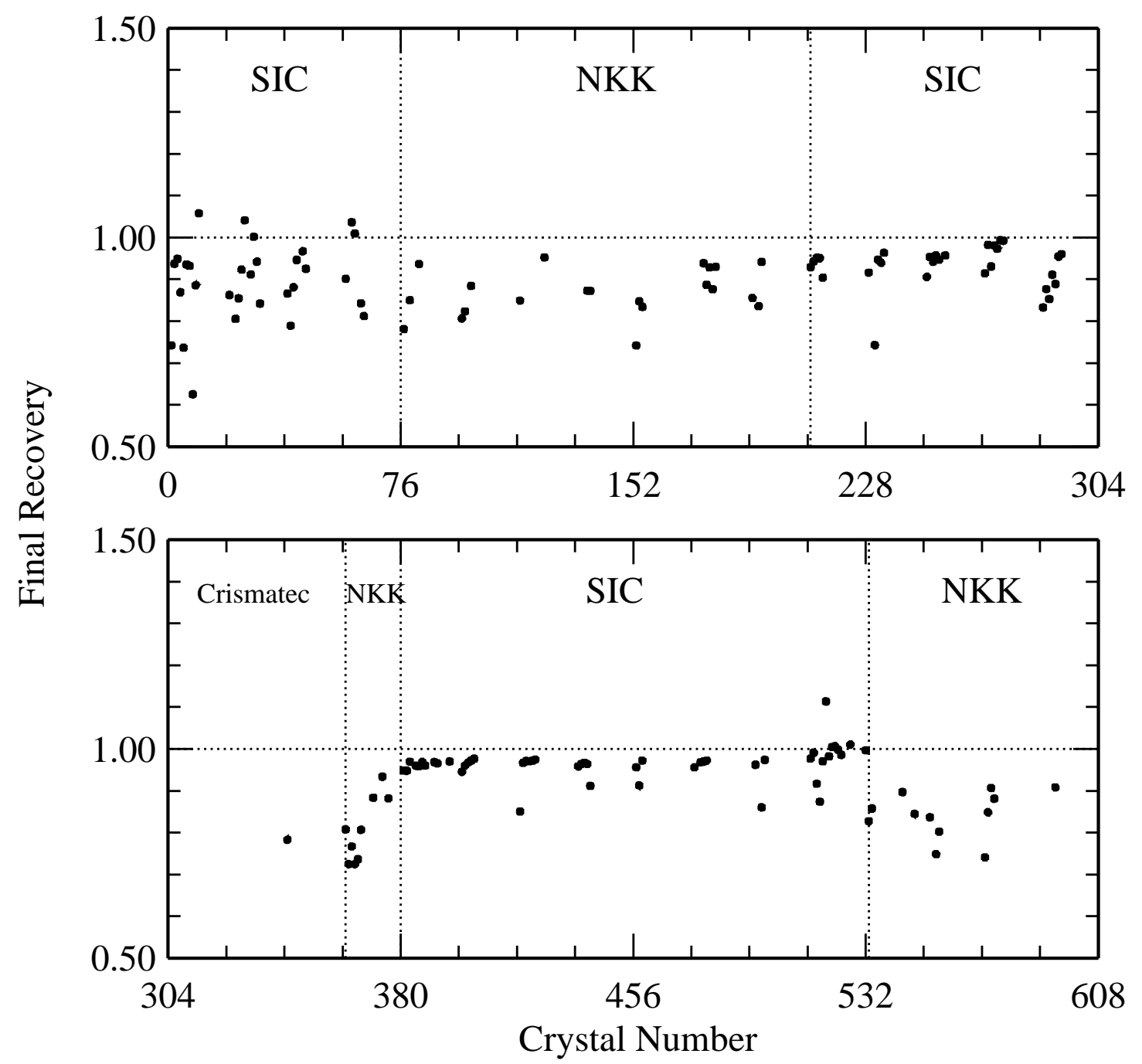

Figure 10: The final recovery of the BGO crystals of different suppliers for the 1989 radiation damage incident. 


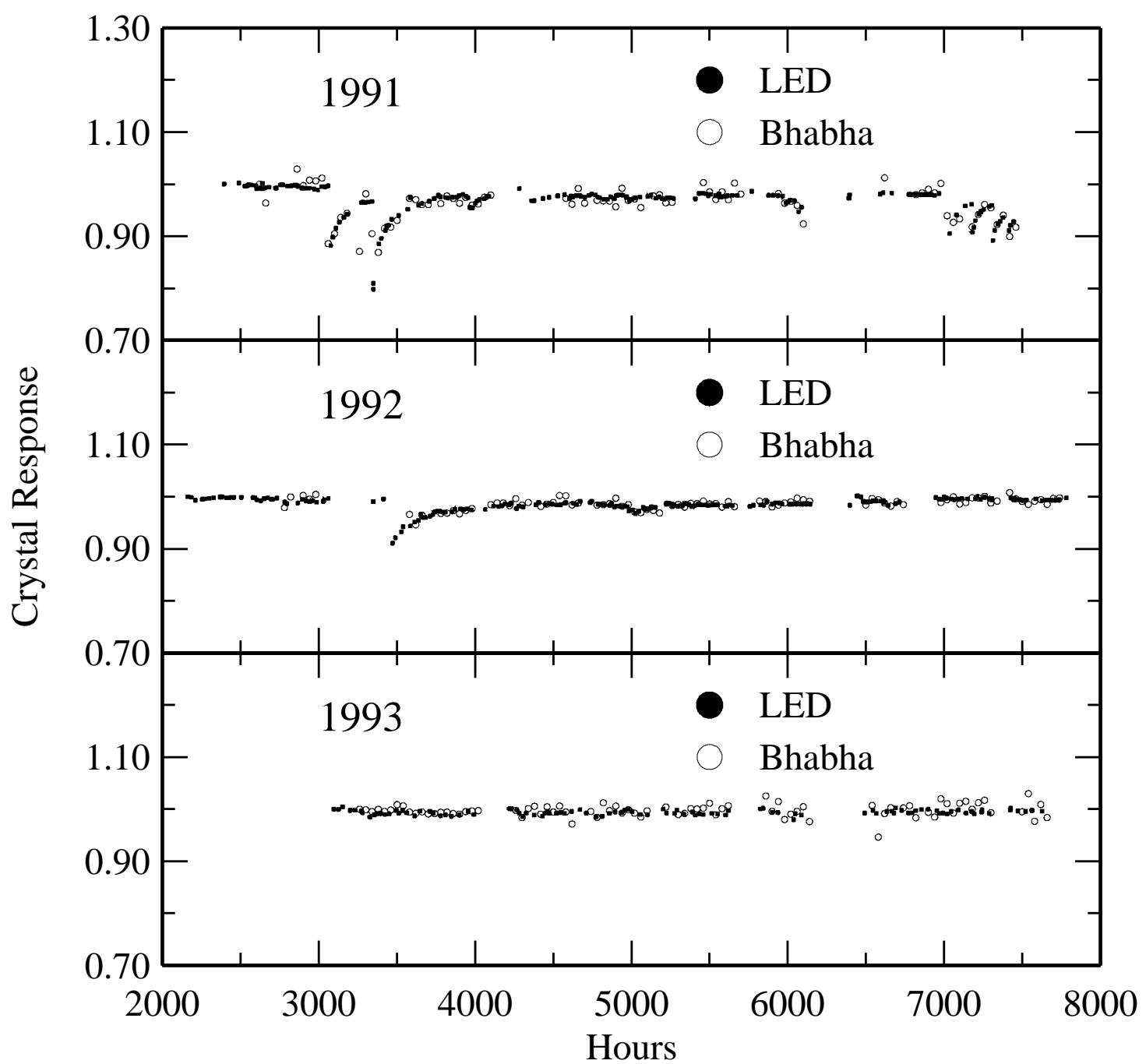

Figure 11: The LED pulse height for crystal 115, normalized to the first run of each year (full dots). The effect of the radiation damage can be easily seen during the 1991 and 1992 run periods. The open circles denote the pulse heights in real Bhabha events. 
the ratio of the energy measured in the trigger FERA to that in the digital readout for each sector, the number of dead crystals, decoding errors and the Bhabha energy spectrum are checked every 100 events to ensure that there are no problems.

\subsection{LEP Luminosity and Background Monitoring}

Special high-impedance, high-gain $(\times 10)$ receivers are used to provide copies of the analog sector energies used for the trigger. The energies in each BGO calorimeter are summed into groups of four sectors to provide four quadrant energies from each side $(-z,+z)$ for background monitoring and continuous measurement of the luminosity (independent of L3 data taking).

A latched discriminator card and a scaler count the number of times each quadrant energy is above an energy threshold of about $25 \mathrm{GeV}$. In addition, back-to-back coincidences are formed to count the number of Bhabha events. Even though such an arrangement is more sensitive to detector and beam offsets than the offline Bhabha selection criteria, a cross-check of this online luminosity and the final offline luminosity shows agreement to within $2 \%$.

A plot of the background rates and luminosity measured using this setup is shown in Figure 12. Variations in the background and the luminosity due to tuning of the machine can be clearly seen. 


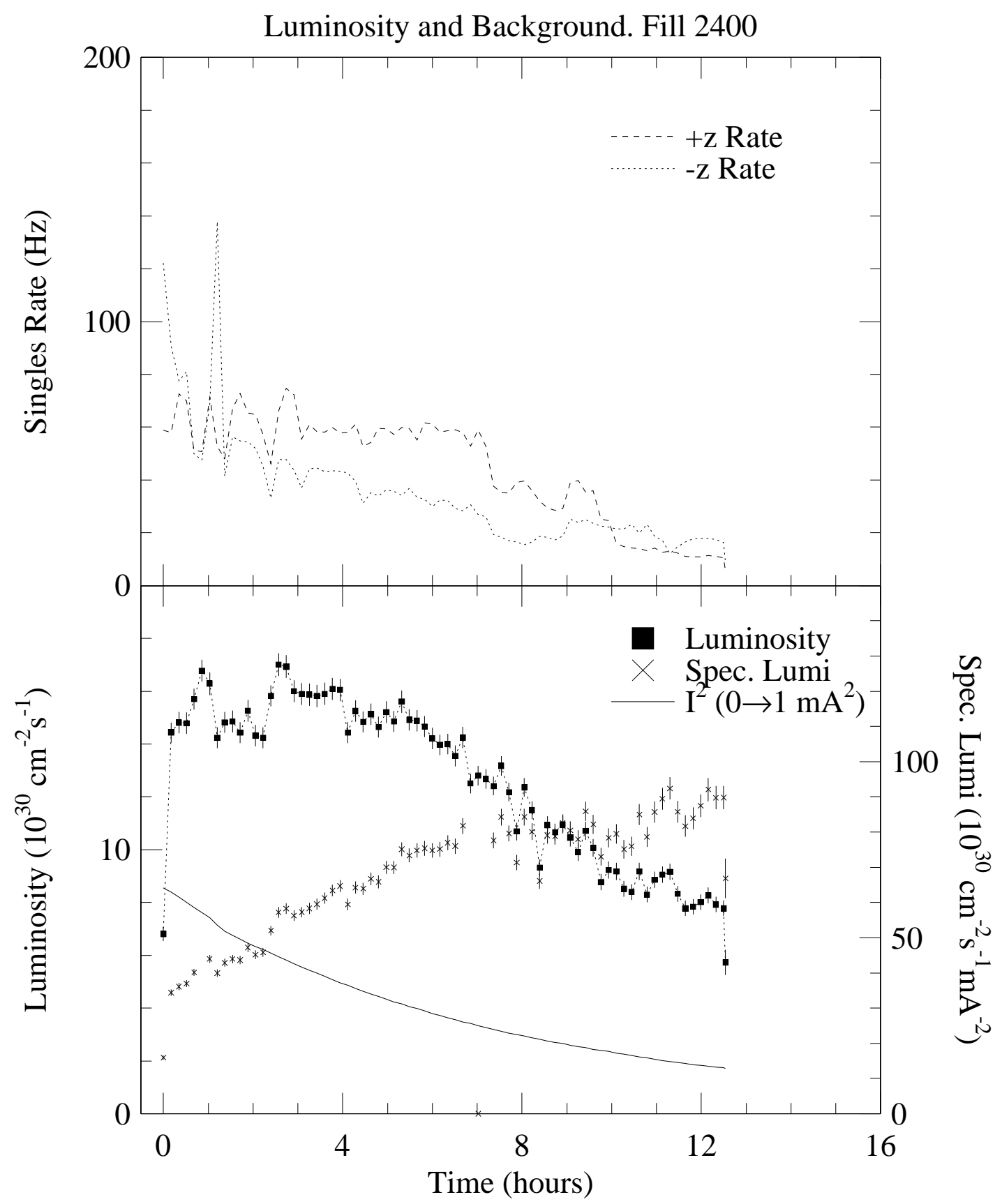

Figure 12: Background rates and luminosity measured online for fill 2400 . The singles rates are the rates for which any quadrant on the $-z$ or $+z$ side has an energy above about $25 \mathrm{GeV}$. The square of the beam current $\left(I^{2}\right)$ and the specific luminosity (Luminosity $/ I^{2}$ ) are also shown. The scale for $I^{2}$ is from 0 to $1 \mathrm{~mA}^{2}$ (not shown). 


\section{The Silicon Tracker}

A silicon strip detector (SLUM) is mounted in front of each BGO calorimeter. On each side the detector has three layers: two layers of strips concentric with the beam axis (" $r$ wafers") to measure the $\theta$ angle of a traversing particle, and one layer of strips perpendicular to the beam axis (" $\varphi$ wafers") to measure its $\phi$ angle. The selection of Bhabha events is improved by the fine granularity of this detector and the accurate knowledge of the detector geometry.

A list of some of the relevant silicon tracker parameters is given in Table 2. Each de-

\begin{tabular}{|lr|}
\hline Distance of central layer from the I.P. & $2650 \mathrm{~mm}$ \\
Minimum radius & $76 \mathrm{~mm}$ \\
Maximum radius & $154 \mathrm{~mm}$ \\
Wafer size in $\phi$ & $24^{\circ}$ \\
$r$ wafer small strips & $64 \times 0.500 \mathrm{~mm}$ \\
$r$ wafer large strips & $16 \times 1.875 \mathrm{~mm}$ \\
$r$ wafer medium strips & $16 \times 1.000 \mathrm{~mm}$ \\
$\mathrm{SiO}_{2}$ insulation between strips & $0.1 \mathrm{~mm}$ \\
$\varphi$ wafer strip size & $0.375^{\circ}$ \\
Layer spacing & $40 \mathrm{~mm}$ \\
\hline
\end{tabular}

Table 2: The general characteristics of the silicon tracker.

tector layer is built out of 16 silicon wafers which have an overlap of $1.5^{\circ}$ in $\phi$. In order to make optimum use of a limited number of silicon channels, a setup has been chosen in which the $r$ wafers contain 96 silicon strips of three different pitches $(64 \times 0.5 \mathrm{~mm}, 16 \times 1.875 \mathrm{~mm}$ and $16 \times 1.0 \mathrm{~mm})$. With this layout the regions close to the fiducial volume cut boundaries have the best granularity. There are 64 channels on each $\varphi$ wafer, giving a total of 8192 channels for the complete detector. Schematic views of an $r$ wafer and a $\varphi$ wafer are shown in Figure 13. There are 5 survey marks on each wafer, so that its position with respect to the other wafers can be optically measured after the assembly.

In most high precision silicon vertex detectors the charge deposited by a particle passing through a silicon wafer is spread over several strips, which means that a resolution significantly smaller than the strip width can be achieved (6-7 $\mu \mathrm{m}$ for $50 \mu \mathrm{m}$ strips, for example). For luminosity determination it is more important to define the fiducial volume accurately, while the spatial resolution is of secondary importance. Relatively large strips are therefore used and every strip is bonded to a readout channel, so that the charge is collected on a single strip. The edges of the strips can then be used to define the fiducial volume.

An overview of the detector is shown in Figure 14. The silicon wafers are glued onto the printed circuit boards containing the first level of the readout. This first-level readout is based on the AMPLEX [9] chip. For each layer the printed circuit boards are mounted on both sides of a Stesalit plate, which allows the wafers to overlap in $\phi$. From the printed circuit board, differential analog signals go to the counting room (120 meters away from the detector) where a readout system based on a (VME) flash analog to digital converter (FADC) [10] is installed. The readout system suppresses any channels that are not above threshold and then transfers the data to a Fastbus memory (Lecroy 1892), where it is merged with the rest of the L3 data. 

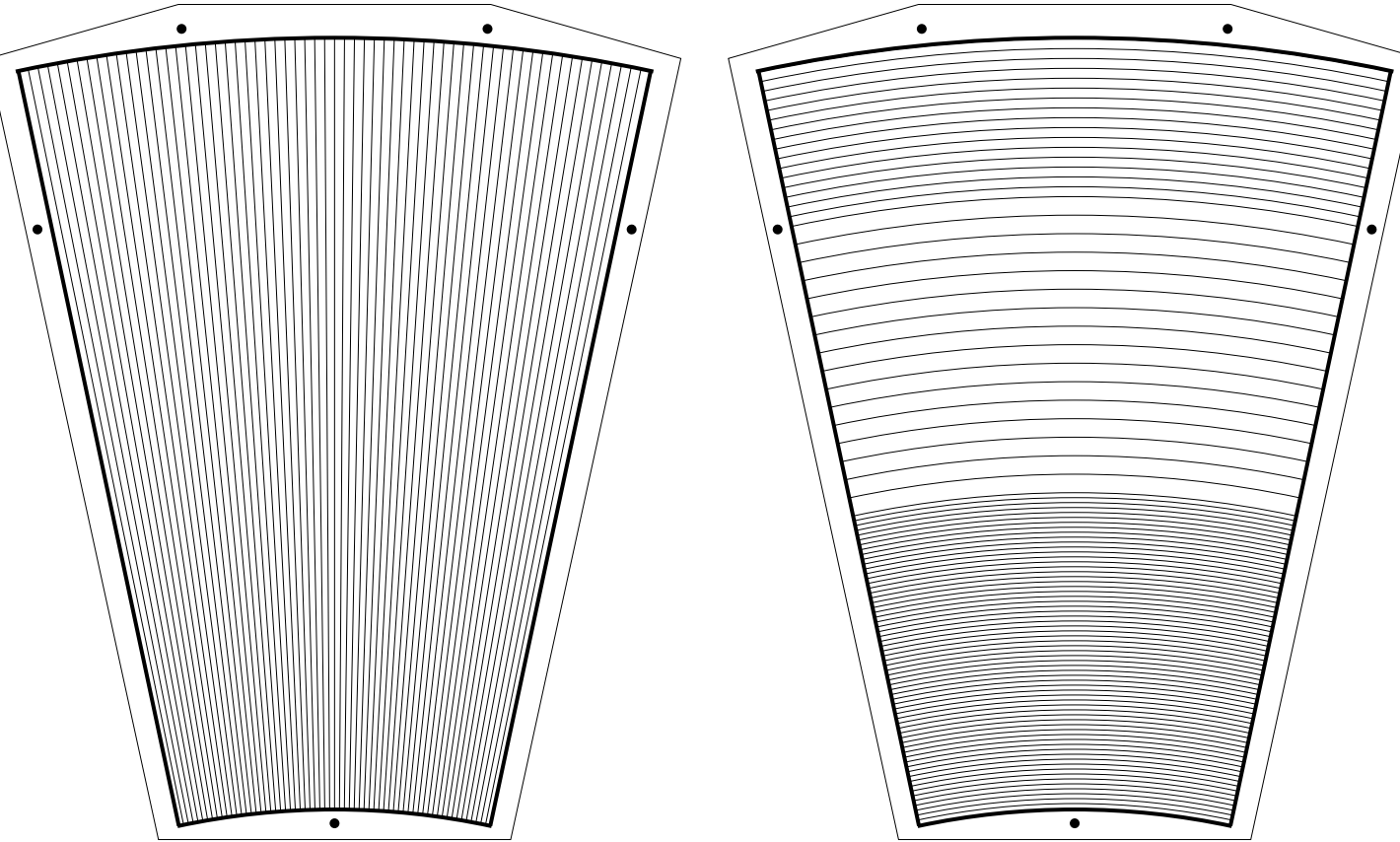

Figure 13: Schematic view of a $\varphi$ and an $r$ wafer. The 3 different $r$-wafer pitches can be seen. The positions of the 5 survey marks on each wafer are indicated by dots.

\subsection{Beam Pipe}

Since the silicon tracker is sensitive to individual particles, it is desirable to minimize the amount of material in front of the detector in order to reduce the amount of showering before the silicon. The most critical area is the region in which the fiducial volume cut is made. In order to achieve this, a specially shaped beam pipe was installed on the $+z$ side of the interaction point (see Figure 1). Installation of an identical beam pipe on the $-z$ side, although desirable, was not possible as it would interfere with the installation jig of the L3 microvertex detector. Figure 15 shows the amount of material as a function of the polar angle of the scattered particles both for the $-z$ and $+z$ setup. The advantage of the $+z$ beam pipe is clearly seen in the lower amount of material around $\theta$-angles 30 to $40 \mathrm{mrad}$, i.e. the region where the fiducial volume cut is made. A comparison of silicon hit multiplicities for the different beam pipe thicknesses and different amounts of material is given in Figures 28 and 29.

\subsection{Silicon Manufacturing and Assembly}

The silicon wafers were manufactured by Micron Semiconductor [11]. The layout was made with an accuracy of $1-2 \mu \mathrm{m}$, more than adequate for our needs. The wafer thickness is $300 \pm 20 \mu \mathrm{m}$, leading to an expected signal of about $25000 \mathrm{e}$ for a minimum ionizing track. There is a $100 \mu \mathrm{m}$ insulating $\mathrm{SiO}_{2}$ layer between each strip. The other main specifications were that the wafers be cut with an accuracy of $30 \mu \mathrm{m}$; the typical leakage current per strip should be $1 \mathrm{nA}$; the total leakage current should be less than $1 \mu \mathrm{A}$; a maximum of $1(2)$ strips on the $r(\varphi)$ wafers are allowed with a leakage current of up to $100 \mathrm{nA}$, while all other strips should have a leakage current of less than $20 \mathrm{nA}$. 


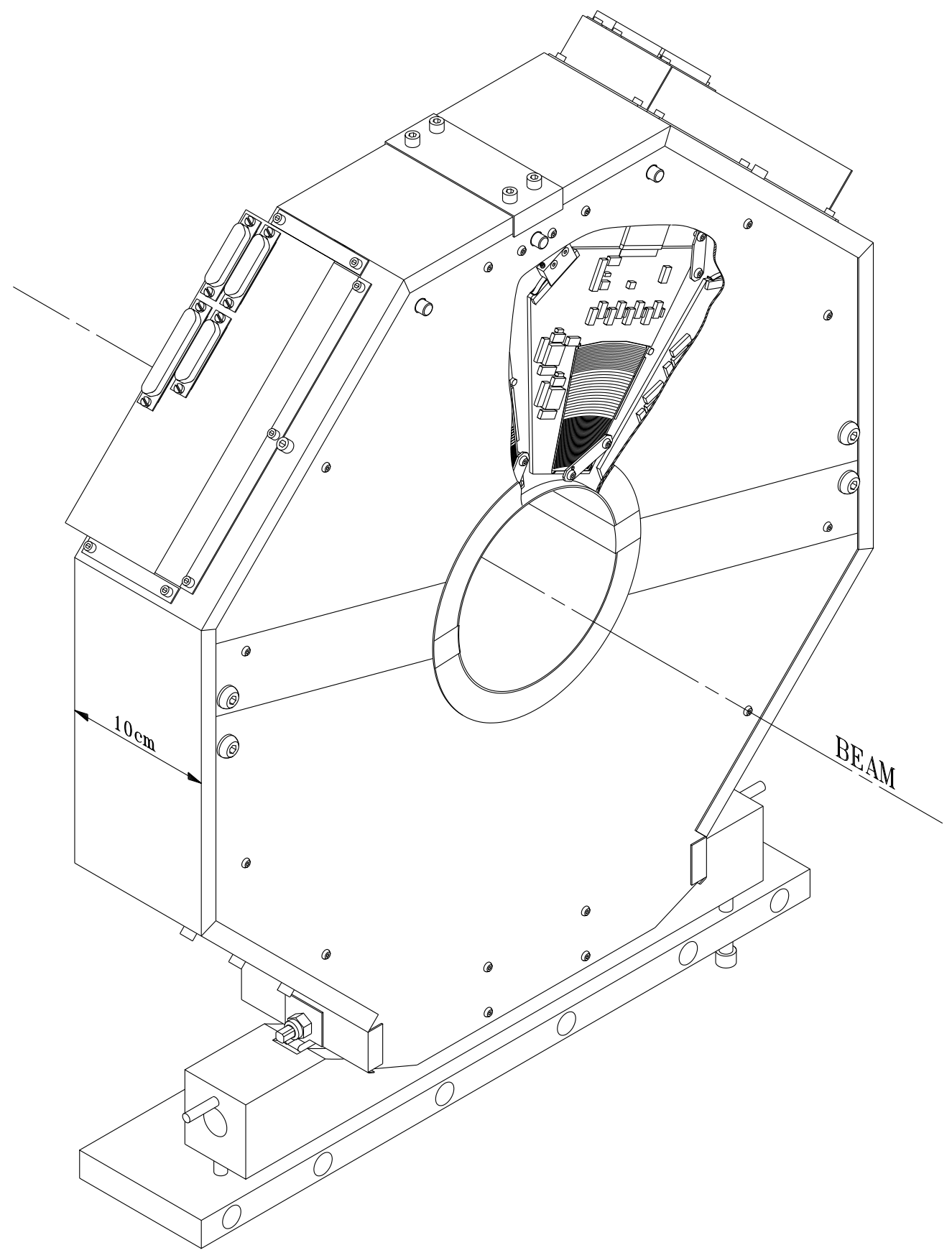

Figure 14: Overview of the silicon tracker. The cutout shows an $r$ wafer mounted on its printed circuit board. The detector support and the connectors for the analog, bias, power and control signals for each half of the detector are also shown. 


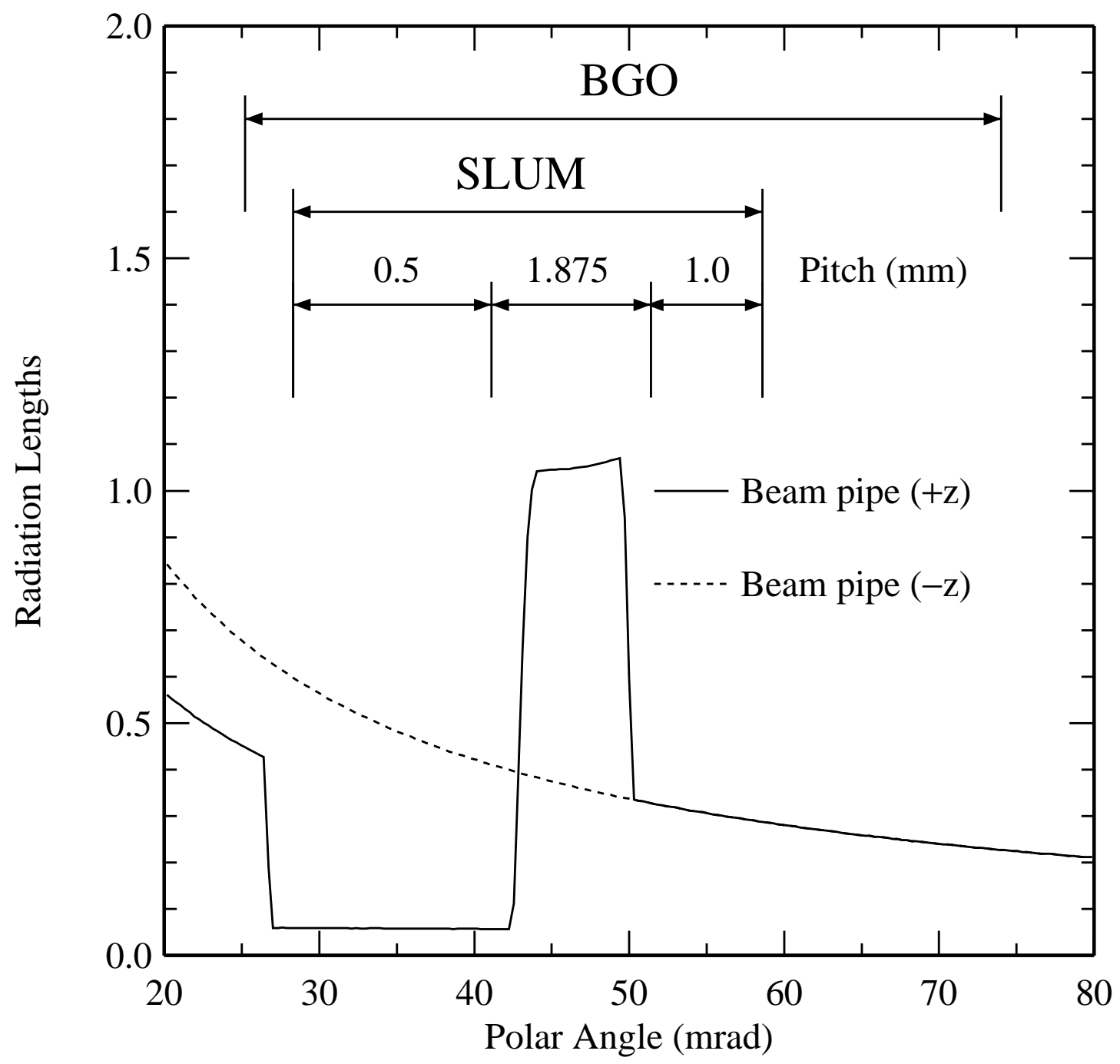

Figure 15: The profile of the beam pipe in radiation lengths on the $-z$ and $+z$ sides. The angular coverage of the BGO calorimeter and the silicon tracker (SLUM) is also indicated. 
Two different coatings were used to protect the wafers: Silox is a scratch resistant silicon dioxide coating, while Polyimide is a scratch and moisture resistant coating. No dependence of the performance of the wafers on the coating has been observed. The effect of these coatings on the leakage currents is discussed in Section 3.4.

The method used to mount the wafers is shown in Figure 16. The wafers were glued onto the printed circuit boards (PCB) using epoxy glue. Each strip is bonded to a gold pad on the printed circuit board using $30 \mu \mathrm{m}$ aluminum wire. The bias voltage connection to the backplane was achieved by bonding from the back of the wafer onto the back of the PCB. Dry

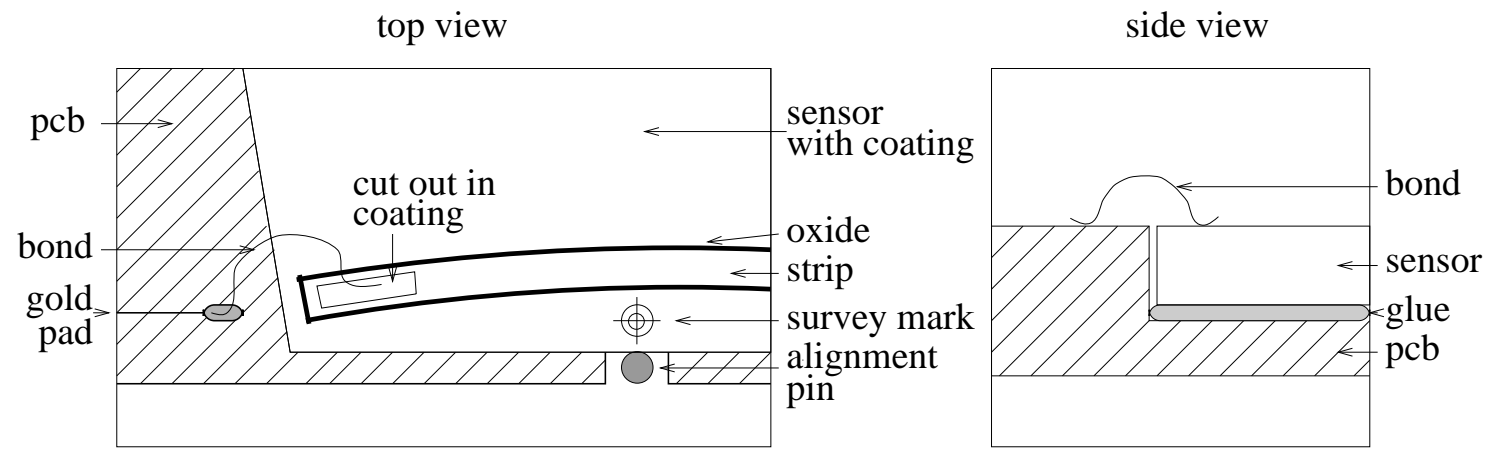

Figure 16: A detailed view of a corner of an $r$ wafer.

nitrogen flows through the assembled detector in order to reduce the sensitivity to changes in the humidity of the air. The operating temperature is $33 \pm 5^{\circ} \mathrm{C}$.

\subsection{Alignment}

The mechanical precision of the wafer support was specified to be better than $30 \mu \mathrm{m}$. This was achieved using $8 \mathrm{~mm}$ thick Stesalit plates as the detector support. The plates are split in the horizontal plane to allow the detector to be installed. Alignment pins ensure that they fit together within the $30 \mu \mathrm{m}$ accuracy and the reproducibility of the assembly was found to be good to $3 \mu \mathrm{m}$.

Rather than positioning the silicon wafer on the PCB with high precision, part of the board was milled away to allow the silicon to touch 3 stainless steel alignment pins. These pins were inserted into the Stesalit plates to the nominal $30 \mu \mathrm{m}$ accuracy. The wafer and $\mathrm{PCB}$ assembly was pushed against the alignment pins and clamped in place. Eight wafers are mounted on each side of the plate to give a $1.5^{\circ}$ overlap in $\phi$ between adjacent wafers.

After assembly, the positions of the silicon wafers were measured using an optical bench. A photograph of one detector during the measurement is shown in Figure 17. The position of the survey marks and of the boundary between the first and second $r$ strips were measured for each plate, with an accuracy of $3 \mu \mathrm{m}$, before the detector was fully assembled. After 1 year of operation the detector was removed, reassembled and the position of the $r$ wafers that are visible when the covers are removed $(50 \%$ of the total) was checked. The radii of the silicon wafers reproduced the previous measurements to within $4 \mu \mathrm{m}$, consistent with the measured reproducibility of the alignment pins used to connect the two half-plates, and with the accuracy of the measuring machines.

The r.m.s. deviation of the position of the wafers from a circle is $26 \mu \mathrm{m}$, consistent with the accuracy of the cutting of the wafers and the positioning of the alignment pins. The average 


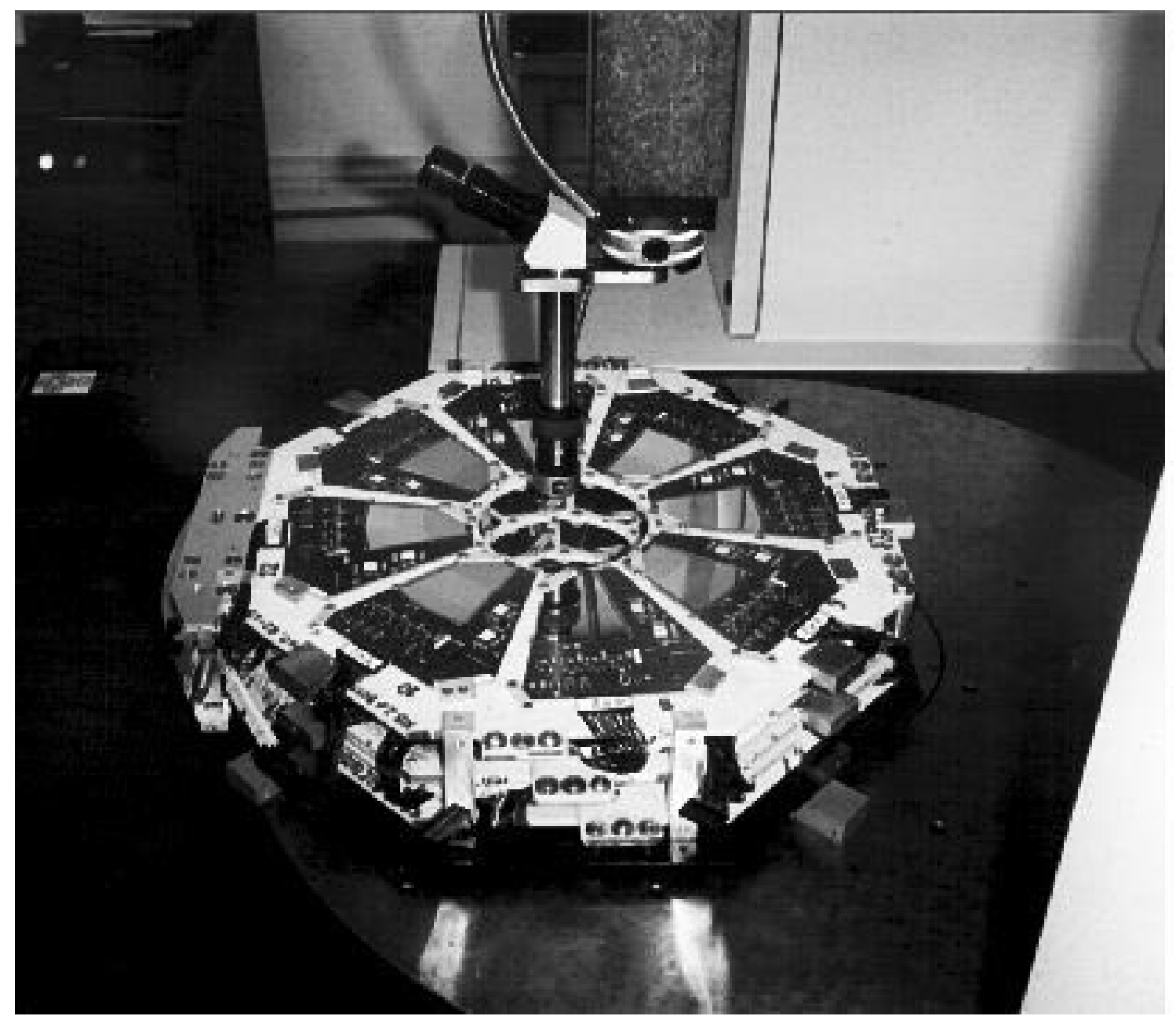

Figure 17: Photograph of a silicon detector during optical survey. The eight $r$ wafers mounted on one side of the Stesalit plate can be seen. The survey head is positioned at the center of the detector. The three layers are visible as are the cables used to connect the $\varphi$ and the $r$ PCBs. 
radius is within $23 \mu \mathrm{m}$ of the nominal radius $(76 \mathrm{~mm})$ and has an r.m.s. of $11 \mu \mathrm{m}$ from layer to layer, showing that the construction of the detector was well within the specifications, both in absolute geometry and in the variation between detectors.

An uncertainty on the absolute radius of the detector of $6 \mu \mathrm{m}$ is assigned. This takes into account the absolute measurement accuracy of the machines, the reproducibility of the assembly and the fact that only half of the $r$ wafers were remeasured.

The radius is corrected for the temperature difference between the measurements and that of detector operation. The expansion coefficient of Stesalit has been measured to be $1.4 \cdot 10^{-5} \mathrm{~K}^{-1}$, in good agreement with expectations. The uncertainty of the operating temperature is included in the systematic error.

\subsection{Leakage Current Measurements}

The performance of the wafers is monitored online by measuring the total leakage current through each wafer. A sudden increase can be an indication of radiation damage. Since the bias voltage is applied through a large resistor which is in series with the silicon wafer, an increasing current leads to a drop in the actual bias voltage and eventually to wafers that are not fully depleted.

During 1993 and 1994 the currents were monitored and the results are shown in Figure 18 for the wafers with the two different coatings, Silox and Polyimide. A clear difference is observed
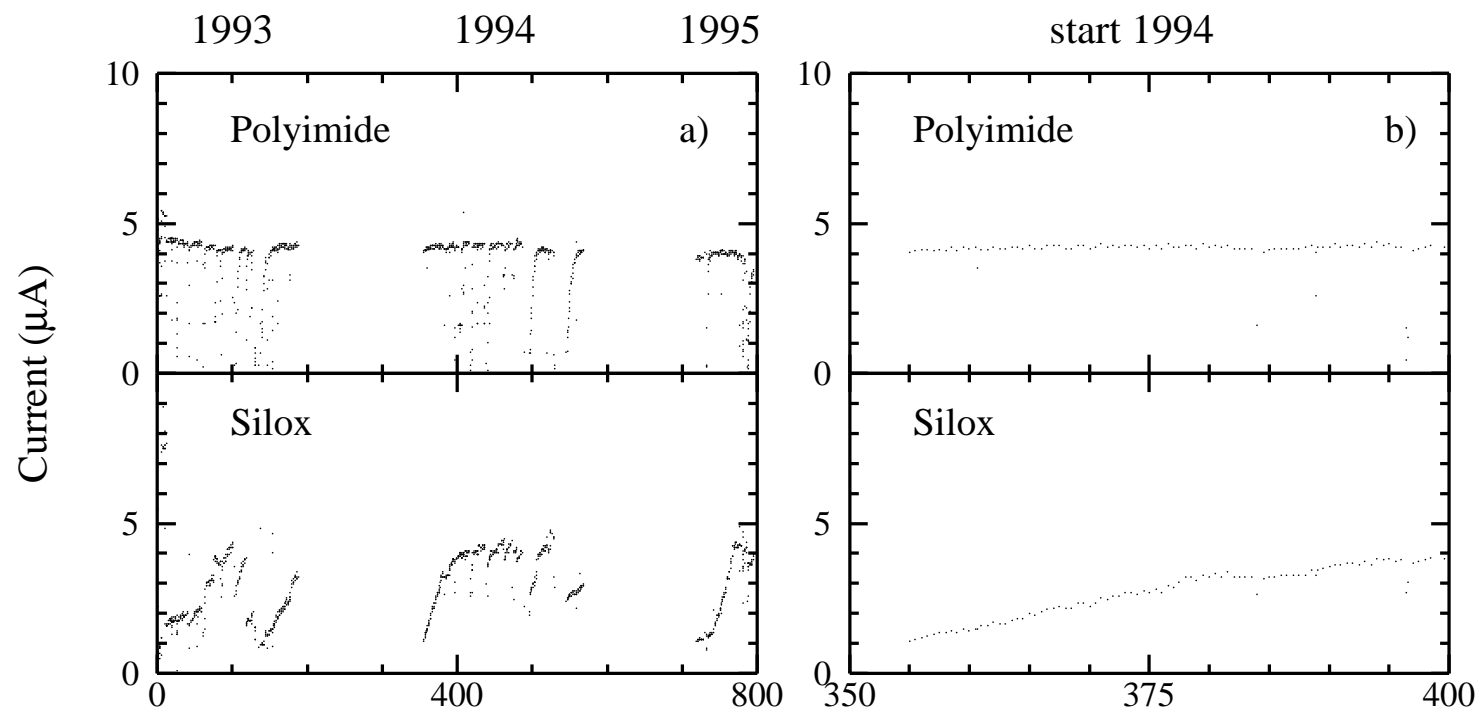

Time (days)

Figure 18: (a) The average leakage currents, as monitored online since the installation of the detector in L3, for the wafers coated with Polyimide (top) and Silox (bottom). (b) A blowup of the period at the beginning of 1994 .

in the behavior of the wafers with the two different coatings. The wafers coated with Silox show a slowly increasing current each time the bias voltage is turned on. While the wafers with the Polyimide coating do not show an increase in time, the current starts at a higher value. 
Although the wafers with different coatings do behave differently, it is hard to conclude which coating is best. For both coatings no increase is observed in the maximum current from year to year, i.e. there is no indication of radiation damage.

To make sure that the position reconstruction is not affected by the increasing currents (a current of several $\mu \mathrm{A}$ through the strips could influence the linearity of the amplifiers), a separate study was performed to determine if the increase is similar in the strip area and in the guard ring. A dedicated setup was used where (Silox-coated) $\varphi$ wafers which are not mounted on printed circuit boards were measured. The leakage current was measured separately in each strip and in the guard ring. The area of the guard ring is comparable with the area of a strip.

The current was measured as a function of time after the bias voltage has been switched on. It took five minutes to measure the current in all of the strips and the measurements were made every five minutes for a period of one week. After the bias had been switched off for one week, the sensor was remeasured in the same way. The results of these measurements can be seen in Figure 19. It is clear that the rise in the current is confined mainly to the guard ring. The response of the detector to a signal generated in the strip area is therefore not affected.
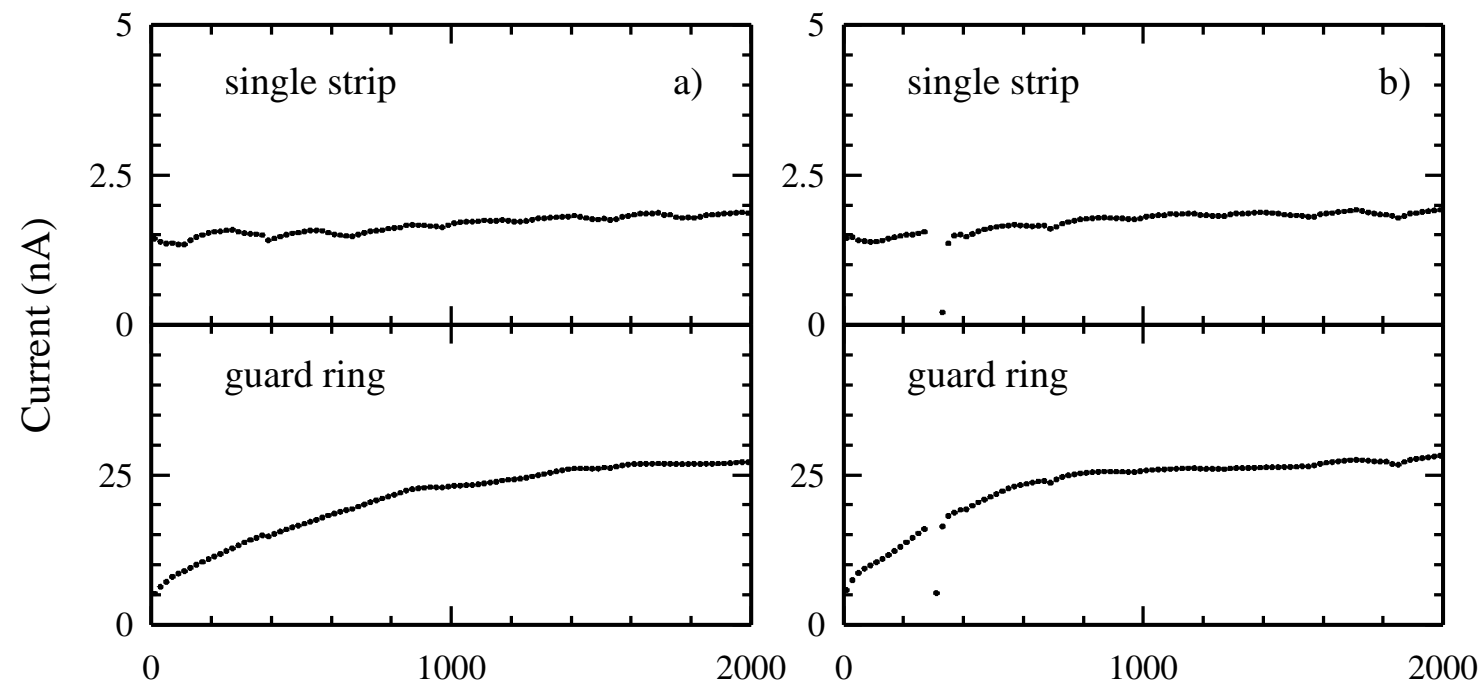

Time (5 min. steps)

Figure 19: (a) The current measured in a typical strip (top) compared with that in the guard ring (bottom). (b) Shows the second measurement 1 week later. The transient behavior is clearly reproducible.

The nature of the leakage current is examined by studying the behavior of the current as a function of the applied voltage. For this purpose the bias voltage is increased from 0 to $100 \mathrm{~V}$ in small steps. The currents for the guard ring and a typical strip are shown in Figure 20.

The leakage current can be divided into a thermally induced current (generation current) and a surface component. The diffusion current, caused by generation of electron hole pairs outside the depletion layer, is neglected. The generation current is proportional to the size of the depletion volume and therefore proportional to $\sqrt{V_{\text {bias }}}$. When the detector reaches full depletion the thickness of the depletion layer no longer increases and the generation current 


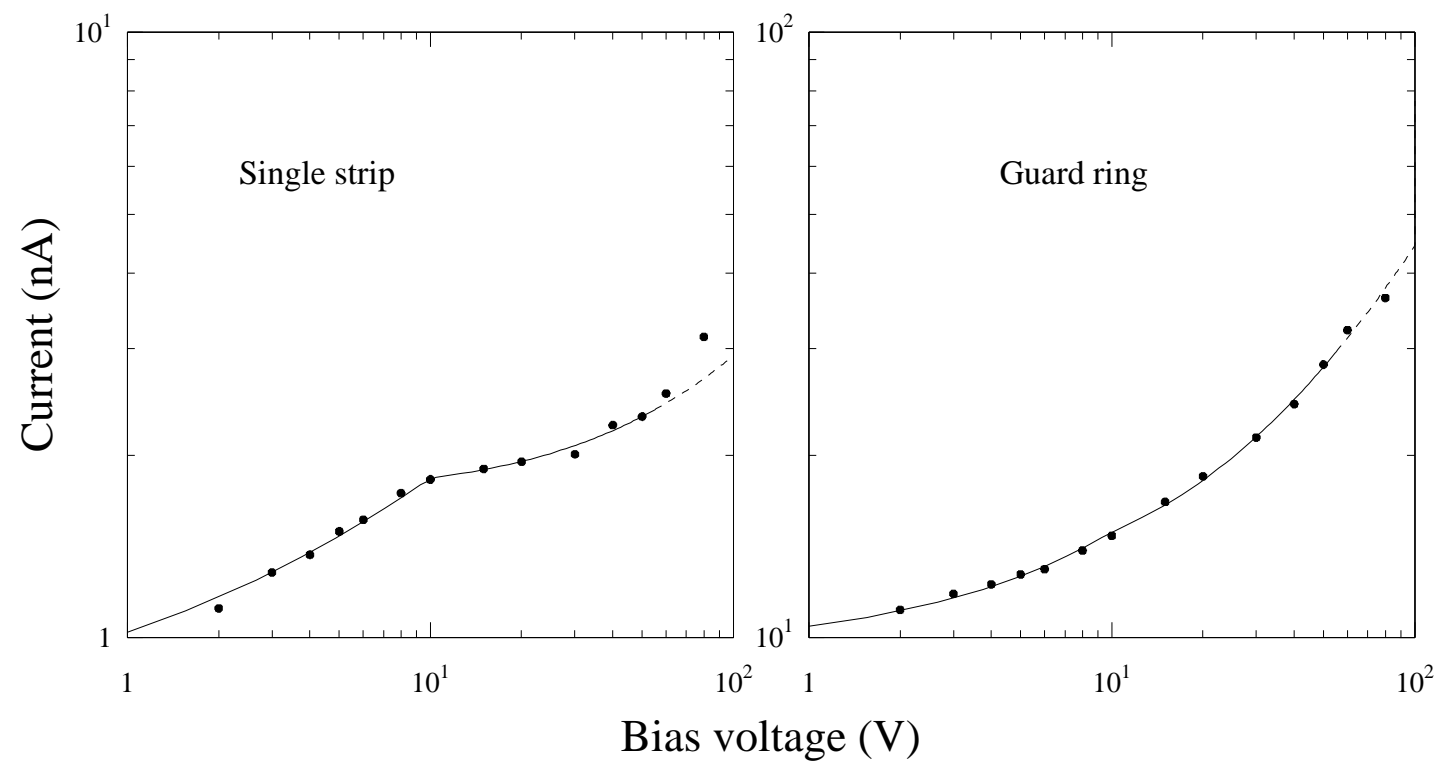

Figure 20: The current as a function of the applied bias voltage (a) through a single strip and (b) through the guard ring. The solid line represents a fit to the data points.

remains constant. The surface current mainly runs around the edges of the sensors and through the protective layer on the surface. This current is dependent on environmental conditions such as humidity and temperature and its behavior is not well understood.

To quantify the effects of the surface current a fit is made using the following equation for the total observed leakage current:

$$
\begin{aligned}
I_{\text {leak }} & =I_{\text {generation }}+I_{\text {surface }}+\text { offset } \\
& =a \cdot \sqrt{V_{\text {bias }}}+b \cdot V_{\text {bias }}+c .
\end{aligned}
$$

The assumption is made that the surface current increases linearly with the applied bias voltage from 0 to $100 \mathrm{~V}$. The offset is introduced to absorb the offsets due to the measuring device. A separate fit is made to the measured currents of a strip and of the guard ring. The generation current is kept constant in the fit, once full depletion has been reached. For this sensor the depletion voltage was measured to be $10 \mathrm{~V}$. From the fit results (shown in Figure 20 and Table 3) it is concluded that the behavior of the current in the guard ring is dominated by surface effects. In the strips the contribution of the surface component is negligible (of the same order as the offset). Using the constants determined from the fit to determine the current at $50 \mathrm{~V}$ (normal operation), the contributions of the two components are as shown in Table 3.

\subsection{Bias Voltage Supply}

The silicon wafers are reverse biased using a $100 \mathrm{~V}$ supply which has a maximum current output of $0.5 \mathrm{~A}$. The readout electronics are powered by two $5 \mathrm{~V}$ linear power supplies. The power fanout system is designed such that the bias voltage for each wafer can be individually switched on/off and adjusted and the leakage current can be monitored. The voltage for all wafers was 


\begin{tabular}{|c|c|c|}
\hline & Single strip & Guard ring \\
\hline$a$ & 0.33 & 0.73 \\
$b$ & 0.01 & 0.33 \\
$c$ & 0.68 & 9.4 \\
\hline \hline \multicolumn{3}{|c|}{ Current at $50 \mathrm{~V}$, in $\mathrm{nA}$} \\
\hline$I_{\text {generation }}$ & 1.0 & 2.3 \\
$I_{\text {surface }}$ & 0.5 & 16.5 \\
\hline
\end{tabular}

Table 3: Results of the fit for the voltage dependence. The overall error on the current measurement is about $0.2 \mathrm{nA}$. The contributions of the generation and the surface currents at a bias voltage of $50 \mathrm{~V}$ are also given. Note that the generation current is assumed to be constant once the full depletion voltage $(10 \mathrm{~V}$ in this case) is reached.

initially set to $50 \mathrm{~V}$. Due to a high leakage current the applied voltage for one wafer was increased to $60 \mathrm{~V}$ in 1994.

Figure 21 shows a circuit diagram of a single bias voltage channel. The reverse bias of a wafer can be changed by adjusting the resistor " $\mathrm{RG",} \mathrm{and} \mathrm{the} \mathrm{leakage} \mathrm{current} \mathrm{is} \mathrm{monitored}$ by measuring the voltage $(\approx 0.15 \mathrm{~V})$ across the $100 \mathrm{k} \Omega$ resistor "R4". The resistor "R4" is placed on the bias voltage line rather than the ground return, because the bias supply and the $5 \mathrm{~V}$ supply have a common ground. The voltage across the resistor is buffered using two operational amplifiers (OPAMPs) and read out via CAMAC. The bias voltage to a wafer (as shown in Figure 21 ) can be turned off by applying a small voltage $(\approx 2 \mathrm{~V})$ to the power MOSFET transistor "T5". Figure 21 shows the schematic diagram for the circuit that produces the voltage to power the OPAMP (OP290GP). The values for the resistors and the capacitors are set such that all the supply voltages for the OPAMPs rise and fall with approximately the same time constant $(\tau \approx 3 \mathrm{~s})$ as the bias voltage, in order not to produce a big voltage differential which could damage them. Interlocks are set to protect the system by putting a lower and an upper bound on the expected current/voltages.

\subsection{Front-end Electronics}

The silicon wafers are read out using AMPLEX [9] chips. One chip, with an input of 16 channels, contains a preamplifier, a shaping amplifier and a multiplexer. The $\varphi$ wafer has 64 channels and is read out with four AMPLEX chips. The $r$ wafer with 96 strips is read out with six AMPLEX chips. In the readout the output of one sector is daisy-chained: one $\varphi$ and two $r$ wafers are read out sequentially at a rate of $1 \mathrm{MHz}$ from $4+6+6$ AMPLEX chips, which produces a train of 256 pulses, representing 256 channels. The full detector consists of 16 sectors on each of the two sides $(-z,+z)$. In total 8192 strips are read out.

The AMPLEX chip has two different modes. In tracking mode, the input charge is collected. In hold mode, the charge is stored on a capacitor until either the channel is read out or the AMPLEX is switched back to tracking mode. The shaping amplifier has a typical shaping time of $700 \mathrm{~ns}$. Thus the track-to-hold switch is made about $700 \mathrm{~ns}$ after each bunch crossing.

The AMPLEX chip is switched back to track mode when a full readout cycle is completed in order to be ready for the next event. The register is cleared so that it points to the first channel again. The timing of the signals concerning the track/hold switch and the clear pulse 

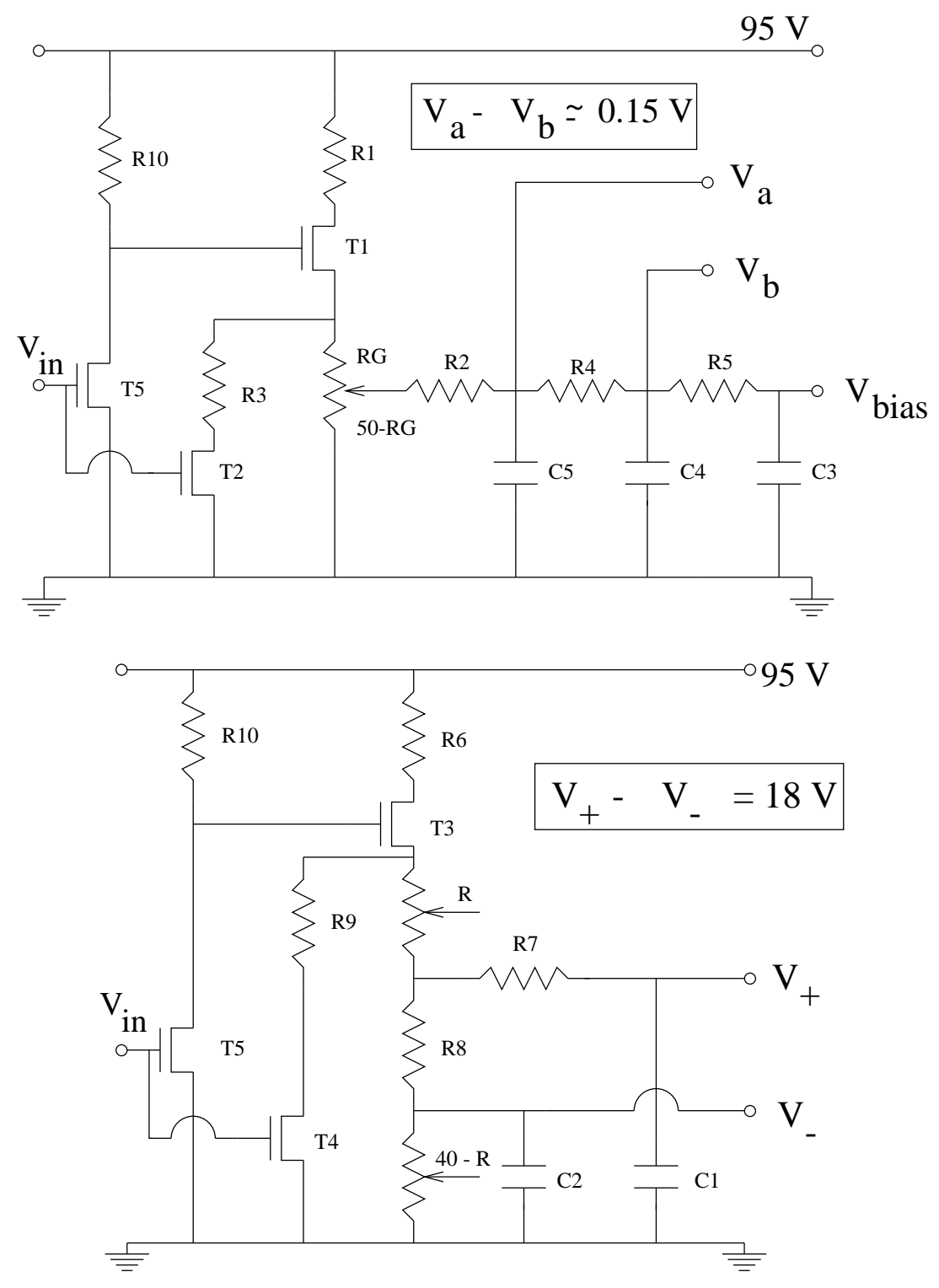

\begin{tabular}{|l|l|l|l|l|l|}
\hline Resistors $(\mathrm{k} \Omega)$ & $\mathrm{R} 1=2$ & $\mathrm{R} 2=2$ & $\mathrm{R} 3=50$ & $\mathrm{R} 4=100$ & $\mathrm{R} 5=2000$ \\
\hline Resistors $(\mathrm{k} \Omega)$ & $\mathrm{R} 6=2$ & $\mathrm{R} 7=2$ & $\mathrm{R} 8=10$ & $\mathrm{R} 9=50$ & $\mathrm{R} 10=100$ \\
\hline Capacitors & $\mathrm{C} 1=2.2 \mathrm{pF}$ & $\mathrm{C} 2=1 \mu \mathrm{F}$ & $\mathrm{C} 3=150 \mu \mathrm{F}$ & $\mathrm{C} 4=68 \mathrm{nF}$ & $\mathrm{C} 5=100 \mathrm{nF}$ \\
\hline
\end{tabular}

Figure 21: Bias voltage circuit and the circuit to power the operational amplifiers. Note that resistor "R10" and transistor "T5" are common to both circuits. The values of the resistors and capacitors are given in the table. 
is critical. The timing scheme is discussed in more detail below.

In the electronic design, the $\varphi$ board plays the role of the master board. All the control signals that the AMPLEX chips need are received by the $\varphi$ board and transmitted to the $r$ boards. In addition, a setup is included on the printed circuit board which allows the combination of printed circuit board and silicon wafer to be tested. A voltage modulation on the bias voltage on the backplane of the silicon wafer is applied, which tests all the strips of the silicon wafers simultaneously. Testing the electronics on the board is done by pulsing either the odd-numbered or the even-numbered input pads of the AMPLEX chips (via capacitive coupling).

\subsection{Readout}

The Flash ADC (FADC) system consists of four functional modules which are installed in separate VME crates. The readout is very similar to the readout of the central tracking chamber of the L3 experiment, the Time Expansion Chamber, TEC [10]. The FADC is used as a fast ADC for many silicon channels, rather than for sampling a slow pulse as it is used for the TEC. A schematic overview of the readout is shown in Figure 22.

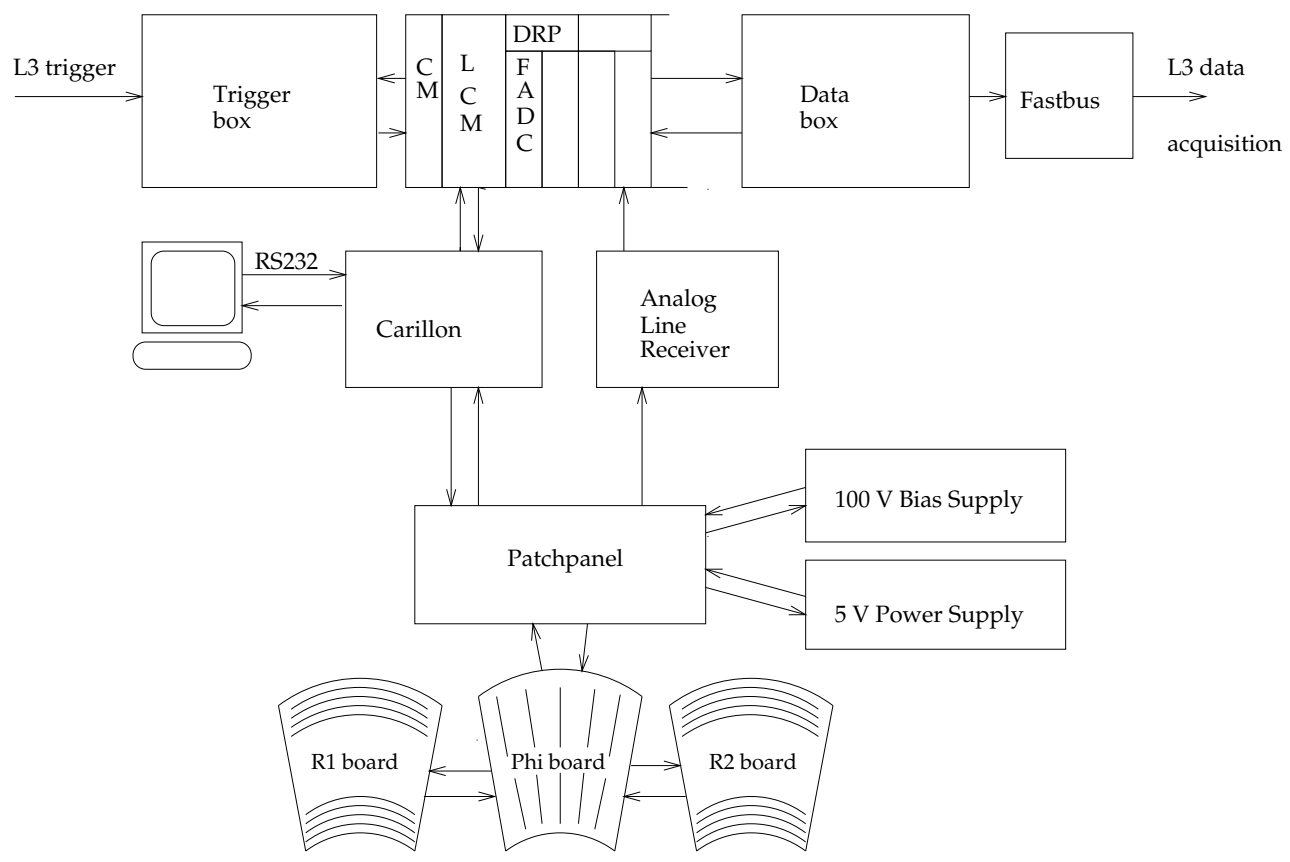

Figure 22: Readout of the silicon tracker.

Analog line receivers (ALR) convert the differential signal that is sent from the front-end readout at the detector into a signal between 0 and $1 \mathrm{~V}$. The output of each ALR channel, which corresponds to one sector (256 strips), goes to a single FADC channel. The FADC has 6 bits, i.e. a full range of 63 counts. The data from the FADC are then passed to a Data Reduction Processor (DRP).

Two FADCs are combined with one DRP in a VME module. The FADC is able to convert a pulse train of analog signals into digital signals, which are synchronized using a clock signal with a frequency of $1 \mathrm{MHz}$ that is sent from the Luminosity Connector Module (LCM). The FADC digitizes the signal on the rising edge of the clock signal. As the FADC system is designed to work with a total of 1024 clocks and L3 requires that each subdetector be readout in less than 


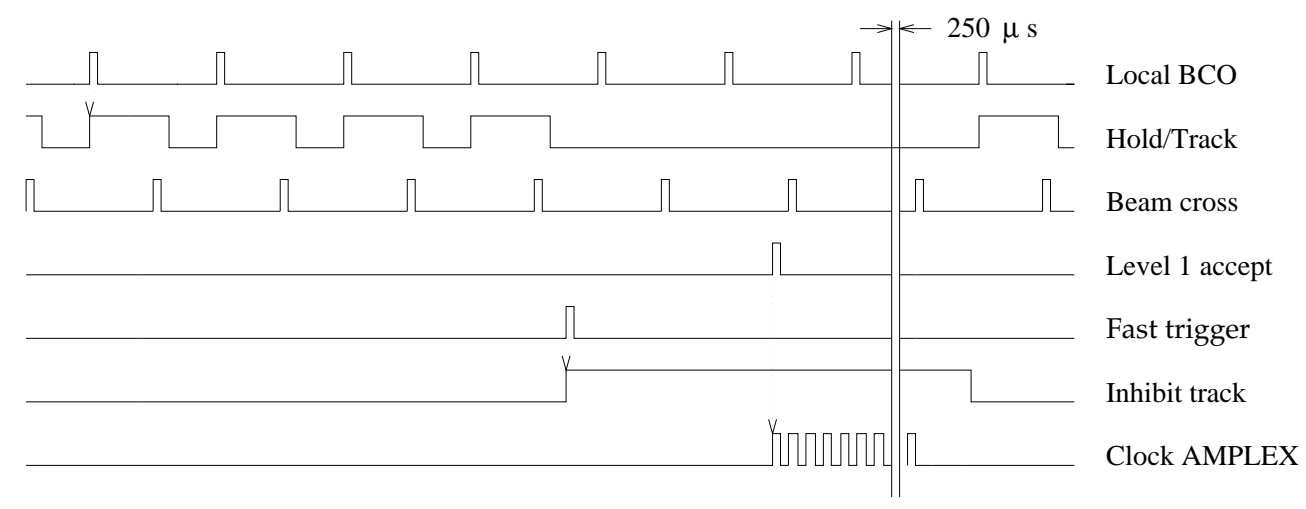

Figure 23: Timing of an event cycle during a global run. The dotted lines indicate that the edge of a given signal sets the signal to which the arrow points.

$500 \mu \mathrm{s}$, the LCM generates a further 768 clocks at high frequency (100 MHz) after the first 256 at $1 \mathrm{MHz}$.

The LCM also provides the necessary interface signals between the readout system and the silicon timing module (Carillon [12]). The Carillon controls the timing variables and the test pulsing of the detector itself. It uses programmable delays in order to synchronize, among others, the track/hold and reset signals on both sides $(-z,+z)$ of the detector in different run modes. In order to check the detector and the readout, it can send test pulses to the backplane, the odd-numbered strips or the even-numbered strips of the silicon wafers.

The values of the timing delays, the run mode and the different variables in the test mode can be set via a terminal (RS232) line. Programs and values are stored in an EPROM so that the Carillon can work completely stand-alone. After every external reset or power failure, the program which is stored in the EPROM automatically resets all parameters in such a way that the Carillon is ready for global data taking.

A large reduction in the number of channels read out is achieved in the DRP. Only the channels above a certain threshold are read out. To do this the pedestal values and their widths are stored in the DRP modules. Separate pedestal runs, internal to the FADC system, are used to calculate the thresholds. These are checked with data taken through the full readout chain. The threshold is set to 1 count above the pedestal.

The crate master (CM) is responsible for transport of the data from the DRPs to the Databox and for downloading programs and data into the DRPs. When a DRP is finished with processing, its data are sent to the Databox. The Databox then sends the data from the silicon tracker to the Fastbus memory, from which it is read out by the L3 data acquisition. The readout system is controlled by a program, TAROT, running on one of the online Vax computers, which also controls the downloading of the software for the CM and DRP [13].

\subsection{Timing of the Event Cycle}

The important timing signals used in the silicon readout are shown in Figure 23. This description is valid for a situation in which the LEP machine is running with $8 \times 8$ bunches. The time between two bunch crossings in the detector is $11.1 \mu \mathrm{s}$, and the detector has to be switched to tracking mode about $7 \mu$ s before the bunch crossing (see Section 3.9). This switching time is set by the "local BCO" signal, which is generated from the standard L3 "beam cross" signal.

As discussed above, the L3 Level-1 trigger signal from the BGO calorimeter is only available 
about $1 \mu \mathrm{s}$ before the next bunch crossing. Therefore, a special "fast" trigger was built using the analog sum signal from each BGO calorimeter. The trigger was constructed using NIM discriminators rather than the FERA system used for the main trigger, in order to avoid the delay due to the digitization of the signal. The fast-trigger signal is available about $3 \mu$ s after the bunch crossing. The trigger was found to have an inefficiency of $1.2 \%$ in 1993 using the same threshold on each side. The efficiency was improved for the 1994 run by splitting the analog signal and using a high energy threshold on one side and a lower threshold on the other side in the same way as the normal double-tag trigger. The inefficiency was then $0.2 \%$. It should be emphasized that the purpose of this fast trigger is only to decide whether the information from the silicon detector for a bunch crossing should be kept.

The event cycle depends on whether there is an L3 Level-1 trigger or not, and also on whether the fast trigger fired:

- A positive fast-trigger decision and a Level-1 trigger cause the AMPLEX signals to be kept in hold and the detector to be read out.

- A positive fast-trigger decision and a Level-1 abort cause the data for the next bunch crossing to be set to 0 , as the tracking time is too short.

- A positive Level-1 trigger, but no fast trigger, causes the data for the current event to be set to 0 .

- A negative fast trigger and a Level-1 abort mean that the detector is put into tracking mode at the right time and is ready for the next bunch crossing.

\subsection{Pedestals and Noise}

The AMPLEX pedestal depends strongly on the length of time that the AMPLEX is in tracking mode, if this time is less than $8 \mu \mathrm{s}$. A plot of the average pedestal and the r.m.s. of the pedestal values as a function of the time in tracking mode is shown in Figure 24. From this figure it is clear that the AMPLEX should be in tracking mode for at least $7 \mu \mathrm{s}$. For this reason the fast trigger described above had to be built.

The average pedestal and pedestal width for the $-z$ and $+z$ sides as a function of time in 1993 are shown in Figure 25. The improvement in the pedestal width on the $+z$ side in the middle of the year was due to a change in the grounding of the detector.

During the silicon tracker prototyping, the noise was studied in order to identify and quantify the different sources. The noise due to the wafer leakage current is negligible compared to the signal left by a minimum ionizing particle (typically 25000e). The capacitive noise comes from the strip, its trace and the AMPLEX capacitance. The total capacitance is between 30 and $40 \mathrm{pF}$ per channel, which leads to a noise of 1400 to $1800 e$ [9].

The noise was also measured as a function of the bias voltage. This is shown in Figure 26 . It can be seen that the noise is constant once the detector has reached full depletion (about $30 \mathrm{~V})$. The noise measurement can be translated into a total detector capacitance and a silicon strip capacitance. The values are in good agreement with the predictions given above.

For each wafer and each region of the wafer, one strip is always read out. These strips are used to estimate the correlated noise for each type of strip. The correlated noise is largest for the large $r$ strips and is in general proportional to the strip area. After applying the correction, the pedestal width for the large $r$ strips is reduced from typically 1.5 counts to 0.7 ADC counts. 


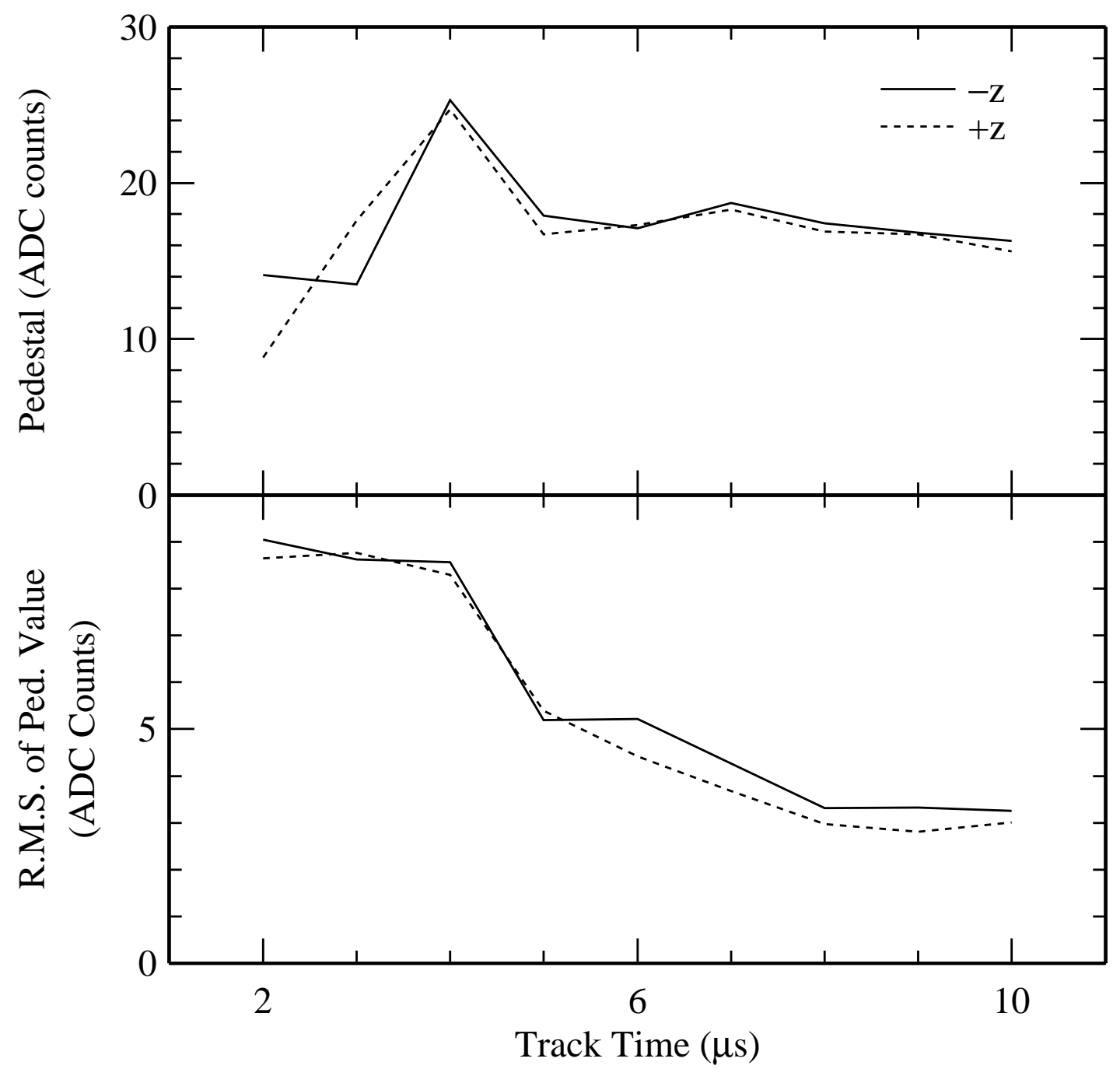

Figure 24: Pedestal values and the r.m.s. of the pedestal values as a function of the time that the AMPLEX is in tracking mode. 


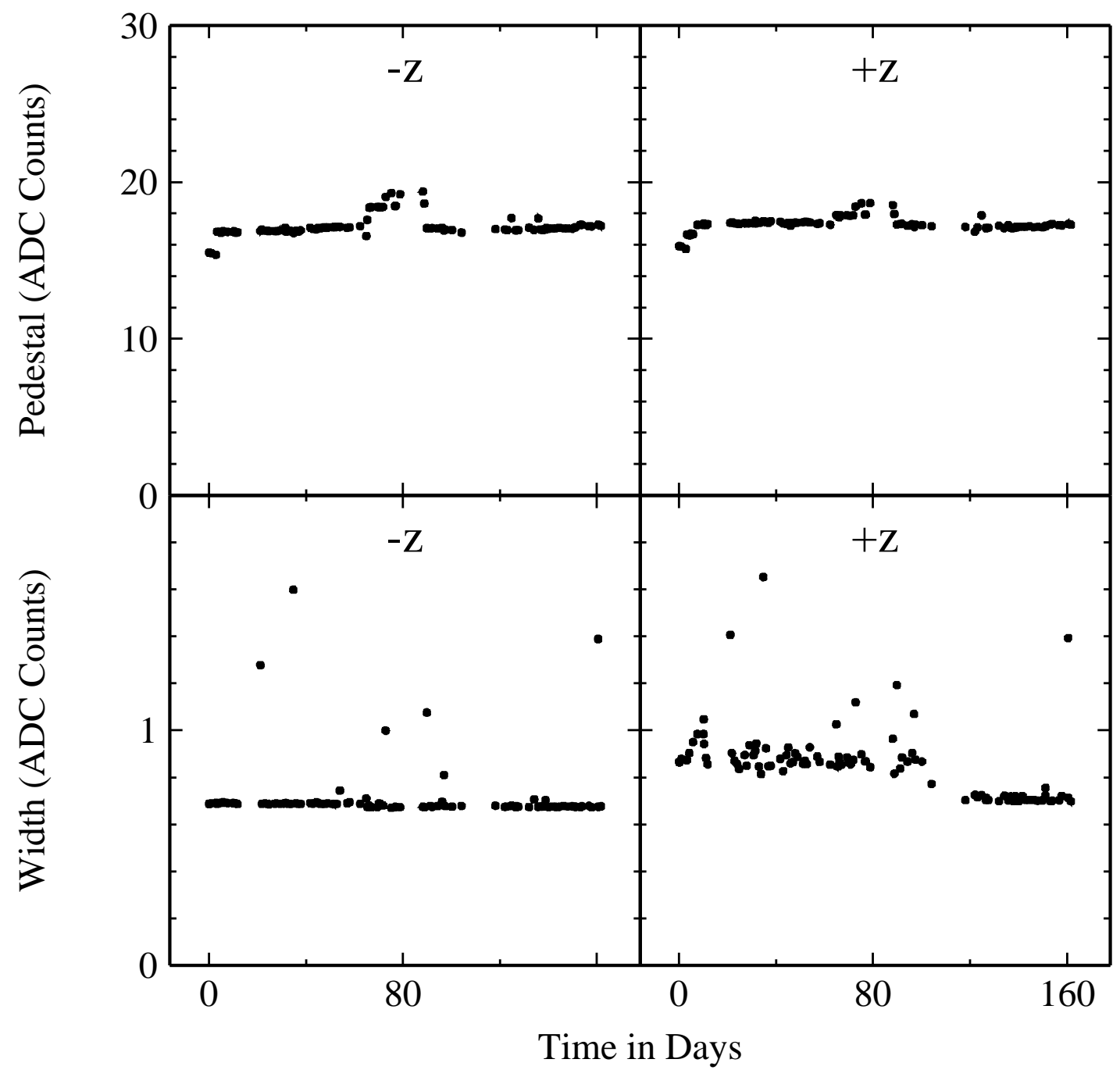

Figure 25: The average pedestal value and r.m.s. pedestal width of the silicon strips during 1993. 


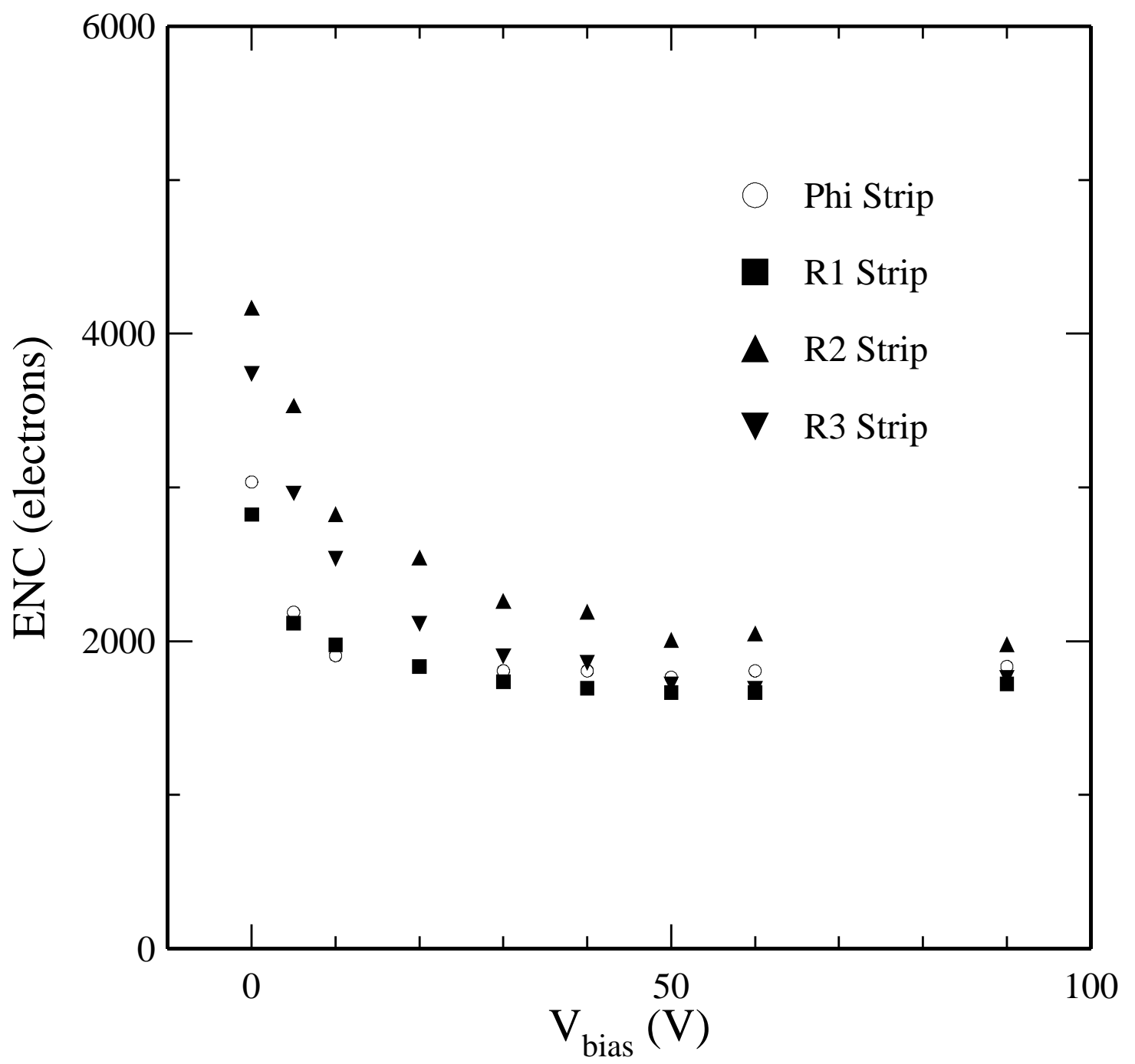

Figure 26: The noise (expressed as the "equivalent noise charge" (ENC) in units of electron charge) as a function of the bias voltage. R1 are the small strips $(0.5 \mathrm{~mm})$, R2 the large strips $(1.875 \mathrm{~mm})$ and R3 the medium-sized strips $(1.0 \mathrm{~mm})$. 


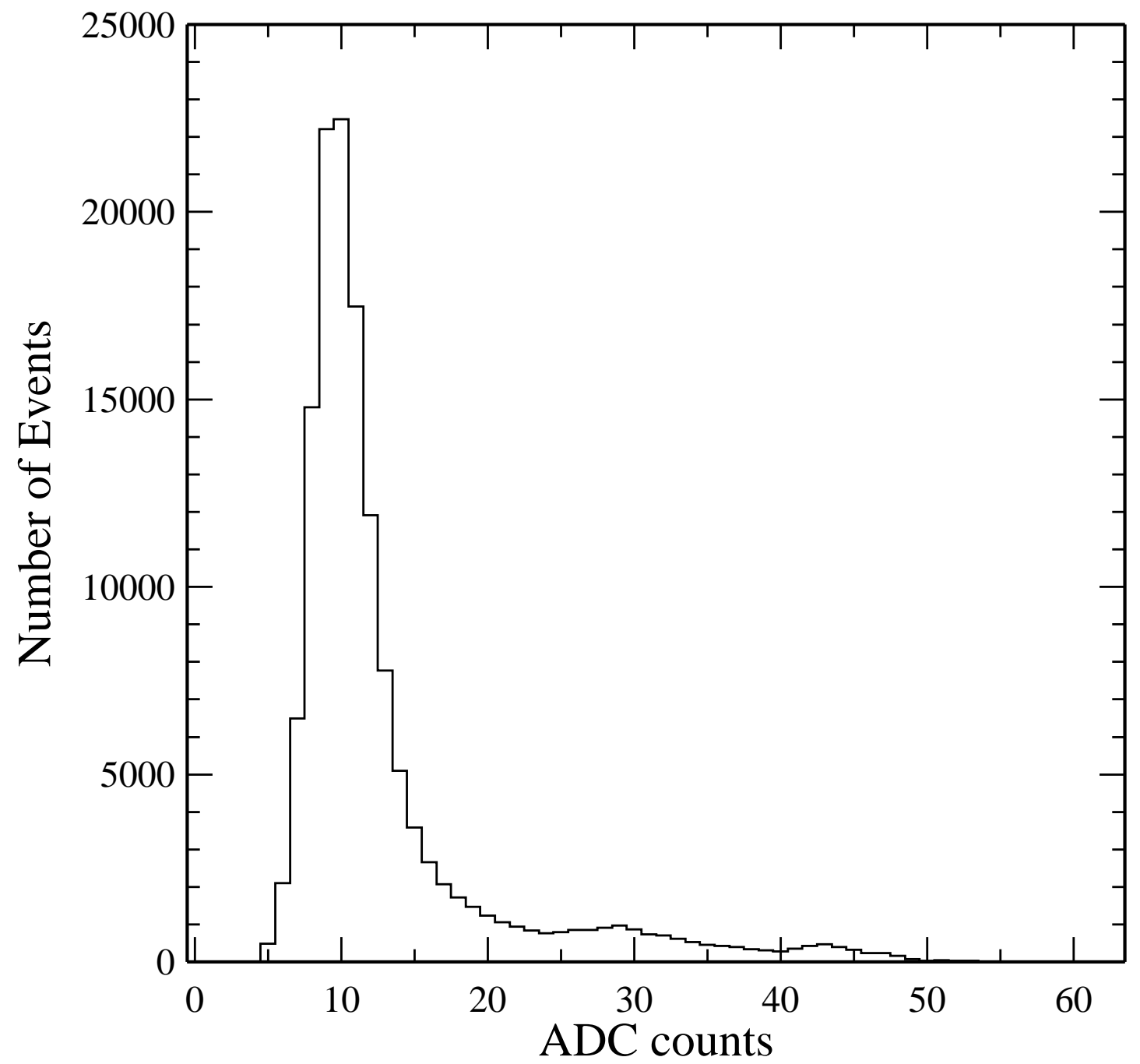

Figure 27: Silicon pulse height distribution (pedestal subtracted). Full scale is 63 counts and the pedestals are typically 20 counts. The peak at about 28 counts corresponds to 3 particles traversing a strip. The enhancement around 43 ADC counts is due to overflows of the FADC.

\subsection{Detector Performance}

Figure 27 shows the pulse height distribution of all silicon strips in ADC counts. Using the fact that the noise is 0.7 ADC counts, the signal to noise ratio is: $\mathrm{S} / \mathrm{N}=14$.

In total 3 dead channels were found in 1993 and 5 dead channels in 1994. This represents a negligible loss.

A silicon strip hit is defined if the following conditions are both valid:

- The strip has a pulse height at least 5 counts above its pedestal value.

- The strip has a pulse height at least its r.m.s. pedestal width above its pedestal value.

The second condition is generally less severe than the first. It serves to prevent noisy strips from continuously causing hits in the detector. 


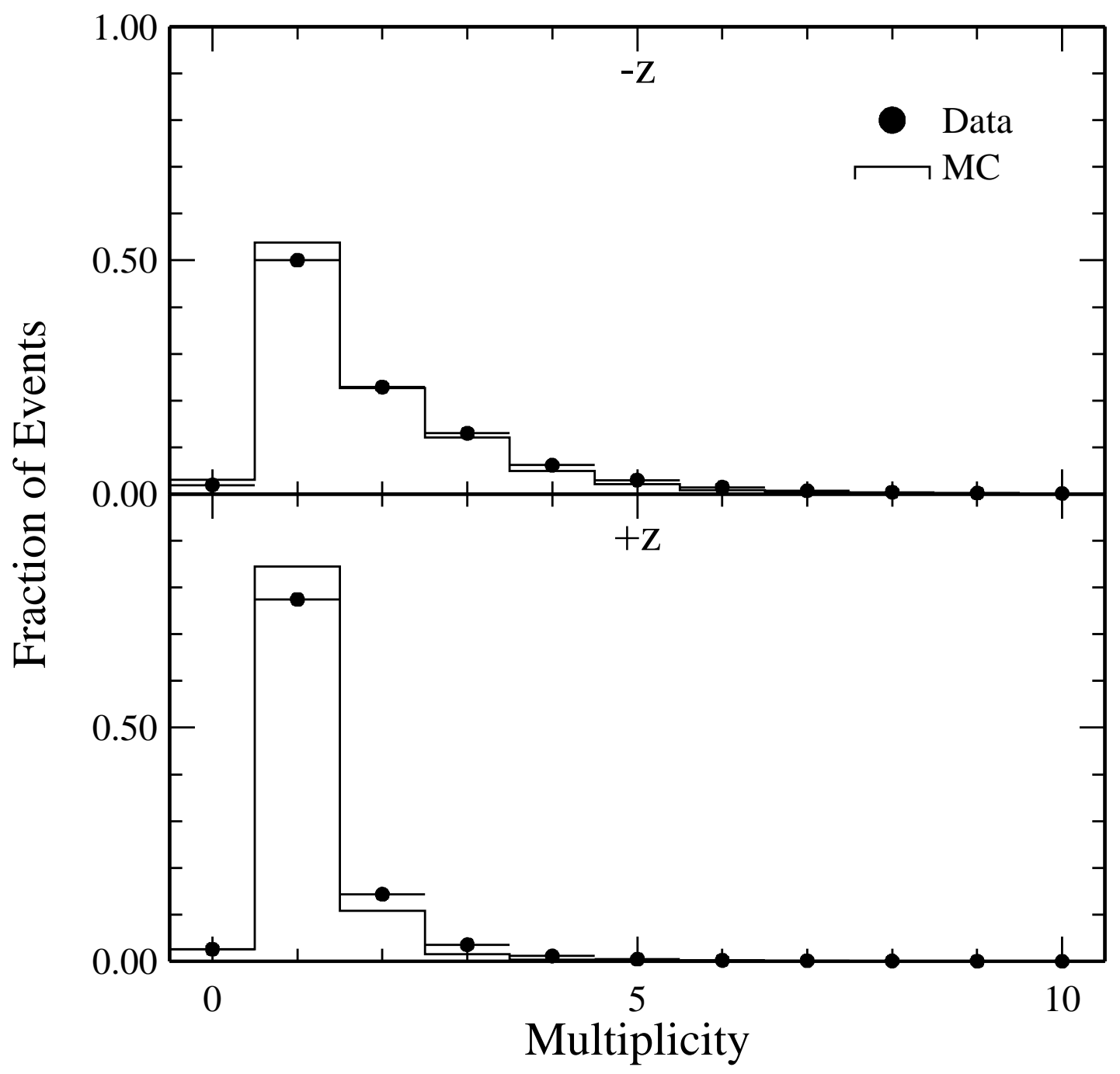

Figure 28: The silicon multiplicity distribution for the small strips in the first $r$ layer on both the $-z$ and $+z$ sides.

The multiplicity distribution of the number of strips that satisfy the two conditions above is shown in Figure 28. Only an area of $(\Delta r, \Delta \varphi)=\left( \pm 5 \mathrm{~mm}, \pm 2.5^{\circ}\right)$ in front of an energy deposition in the BGO is considered. The distributions clearly show a different behavior for the $-z$ and $+z$ sides. The observed multiplicities are strongly correlated with the amount of material seen by a traversing particle before it enters the silicon detector. Figure 29 shows the effect as a function of the amount of material in front of the silicon wafer, as studied in a test beam. From here it can be seen that the amount of scattering caused by the thickness of the beam pipe is consistent with the test beam measurements. Note that the GEANT [4] based Monte Carlo slightly underestimates the multiplicity.

Given that the fast trigger fired, the efficiency for finding a hit in front of the most energetic cluster in the BGO is $98.4 \%$ and $99.2 \%$ for the small $r$ strips on $-z$ and $+z$, respectively. 


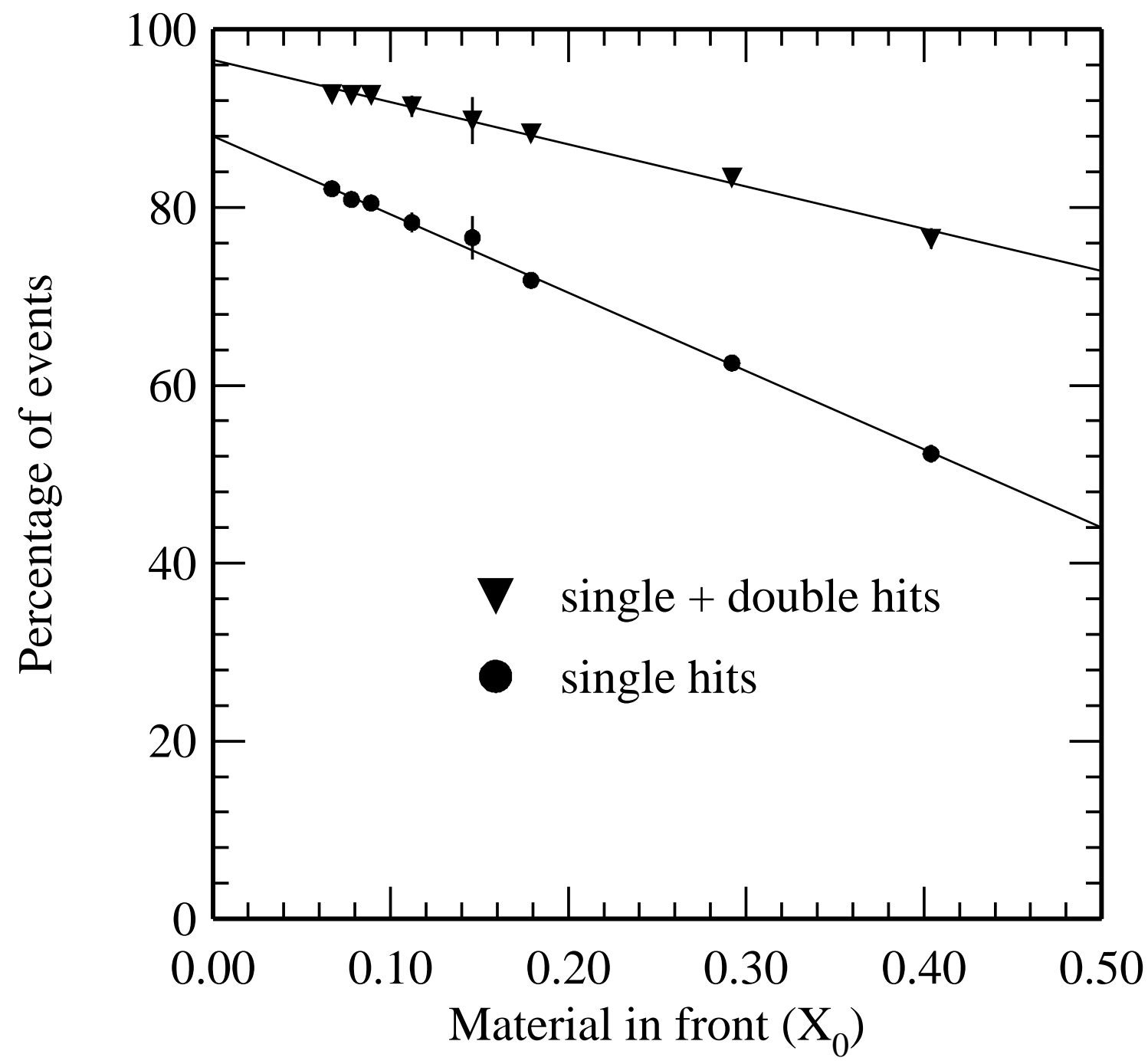

Figure 29: Test beam study of the fraction of events with either one or two silicon strips hit as a function of the amount of material in front of a wafer. The lines are a straight-line fit to the data points. 


\subsection{Coordinate Reconstruction Algorithm}

The direction of a charged Bhabha track is reconstructed in a two-step procedure. In the first step, the track is assigned the coordinates $r$ and $\varphi$ of the hit strip in the detector reference frame. The precision of these coordinates is mainly determined by the precision of the internal geometric alignment of the silicon wafers. In the second step, the track is assigned the coordinates $\theta$ and $\phi$ in the physics reference frame. In this step, the precision of the external geometric alignment of the silicon detector is crucial. The external alignment is discussed in detail in Section 4. The $(\theta, \phi)$ coordinates are used to decide whether the event lies inside the fiducial volume.

Note that the particle hits expressed in detector coordinates are of a discrete nature, governed by the strip granularity of the detector. In the transformation to the physics frame this discrete nature is resolved by assigning each track a specific position on a strip. The hit position is obtained by smearing the position over the strip, taking into account the expected $1 / \theta^{3}$ particle distribution over the surface of the strip and assuming a uniform distribution in $\phi$.

The impact position of the most energetic BGO cluster is used as a starting point for the analysis using the silicon detector. A window of $(\Delta r, \Delta \varphi)=\left( \pm 5 \mathrm{~mm}, \pm 2.5^{\circ}\right)$ is formed around the reconstructed BGO impact point. Only silicon hits which lie within this window are considered.

In the process of determining the impact position of the particle, the following situations can occur:

- There are no hits inside the window. There can be two causes:

- The silicon fast trigger did not fire for this event. This happens for $1.2 \%$ of the Bhabha events in 1993 and $0.2 \%$ in 1994.

- The track which caused the energy deposition did not leave a hit in the detector. This occurs for $1.6 \%$ of the events close to the fiducial volume cut on the $-z$ side and $0.8 \%$ of the events on the $+z$ side.

In both situations the event is not lost, but the impact point of the BGO calorimeter is used for further analysis.

- There is only one strip hit. In this case the position of this strip is assigned to the impact position of the particle. This happens for $52 \%$ of the events close to the fiducial volume boundary on $-z$ and for $80 \%$ on $+z$. The analysis of single hit events is relatively straightforward and has the least danger of introducing biases in the event selection procedure.

- There is more than one hit. This has two main causes:

- A shower in the material preceding the detector (the beam pipe or the detector box) occasionally creates additional particles.

- A silicon strip which has a pedestal fluctuation above the cut value.

This situation occurs for $46 \%$ of events on $-z$ and $19 \%$ on $+z$.

In the analysis no difference is made between events where the BGO impact point is used and events where the silicon hits are used. Thus, it is important that the relative geometrical positions of the two detectors are well understood. 
Three different algorithms have been investigated for events with multiple strip hits, in order to avoid a biased reconstruction of the impact point in the detector.

- Random assignment: the impact point of the particle is assigned at random to one of the strips hit.

- Average assignment: the impact point is calculated as the average of the individual strip hits.

- Pulse height weighted assignment: the impact point is calculated by assuming the multiple hits to be caused by a "mini shower". The impact point is obtained according to a centerof-gravity method.

A Monte Carlo study showed that the position resolution in the pulse height weighted method was best. The luminosities calculated using all 3 methods have been compared. The difference between the the results of the average method and those of the pulse height weighted method is at the $10^{-5}$ level, while the random method gives a luminosity $0.02 \%$ higher. The average method has been used for the other systematic checks given below. Given the excellent agreement with the pulse height weighted method no systematic error is assigned due to the multiple hit algorithm. 


\section{Alignment}

Bhabha events are selected using the calorimetric measurement in the BGO to provide a background free sample of events, and the silicon tracker to select only those events that are contained in a precisely defined fiducial volume. Since the event selection procedure combines the information of the calorimeter and of the tracker, the alignment of the two detectors must be known to a high precision. This alignment is done using the Bhabha events themselves.

\subsection{Detector Positions}

The following must be determined:

- The total distance between the $-z$ and $+z$ silicon detectors using survey measurements.

- The position of the BGO modules in the silicon reference frame.

- The transverse position of the silicon with respect to the beam axis.

- The longitudinal position of the silicon with respect to the vertex.

The procedures to determine these quantities are described in the following sections.

\subsubsection{Survey Measurements}

The total $z$ distance between the silicon detectors at the $-z$ and $+z$ sides of the interaction point is obtained by an optical survey measurement. For this purpose the detector is equipped with four mechanical survey rods which allow the position of the silicon to be measured with respect to the L3 reference frame. Due to a mechanical instability of the mounted survey rods, the optimal precision of this measurement $(0.3 \mathrm{~mm})$ was not obtained in 1993 . Furthermore, as indicated in Figure 25, the external cabling of the $+z$ detector was modified during the 1993 run to reduce the pedestal width. This changed the rotation angle of the detector about the $x$-axis. This change in position prevented a direct comparison between the survey measurements before and after the run. The comparison was done after the displacement had been corrected for using the results of the alignment fit discussed below. The precision obtained on the measurement of the total distance between the detectors during the 1993 run was $1.6 \mathrm{~mm}$. The mechanical stability of the survey rod system and of the detector support was improved for the 1994 run. During this run, no detector displacements occurred and a precision of $0.4 \mathrm{~mm}$ on the total distance between the detectors was obtained.

\subsubsection{Positioning of BGO vs. Silicon}

In order to match the geometrical position of the silicon and the BGO detectors, a two-step procedure is used. In the first step, the global position of each BGO module $((-x,-z),(+x,-z)$, $(-x,+z),(+x,+z))$ is determined in the coordinate frame of the silicon detectors. In the second step, an additional alignment correction is applied independently to each of the 16 BGO sectors in both detectors. After alignment, the reconstructed impact position of a particle in the $\mathrm{BGO}$ calorimeter is expressed in the coordinates of the silicon frame. 


\section{Global Fit}

In the global fit, each of the four BGO modules is treated independently. In order to compare the measurement of the silicon tracker with that of the BGO calorimeter, they need to be extrapolated to a common $z$ position. The position used is that of the shower maximum inside the $\operatorname{BGO}\left(z_{S}\right)$. The shower maximum position is determined using the integrated amount of material seen by an electron emerging from the interaction point. This position is mainly determined by the dense BGO material but also, to a somewhat lesser extent, by the material in front of the detectors, i.e. the beam pipe. As is shown in Figure 15, the amount of material seen by a traversing particle differs for the two detector sides. Moreover, it depends on the polar angle of the emerging particle. These effects are considered in the alignment fit by extrapolating the silicon hits $\left(r_{\mathrm{sil}}, \varphi_{\mathrm{sil}}, z_{\mathrm{sil}}\right)$ to a corrected shower maximum position $z_{\mathrm{sil}}^{\prime}$ :

$$
z_{\text {sil }}^{\prime}=z_{S}-\rho \cdot X_{0}^{\text {pipe }}(\theta)
$$

where $z_{S}$ represents the uncorrected position of the BGO shower maximum, $\rho$ the density of BGO and $X_{0}^{\text {pipe }}(\theta)$ the amount of material in the beam pipe as a function of $\theta$, expressed in radiation lengths. The extrapolated silicon coordinates are then obtained according to

$$
\begin{aligned}
r_{\mathrm{sil}}^{\prime} & =\frac{r_{\mathrm{sil}}}{z_{\mathrm{sil}}} \cdot z_{\mathrm{sil}}^{\prime} \\
\varphi_{\mathrm{sil}}^{\prime} & =\varphi_{\mathrm{sil}}
\end{aligned}
$$

These extrapolated silicon hits are used to perform a $\chi^{2}$ minimization of the distance between the $\mathrm{BGO}$ and silicon points where the following offsets of a BGO half-detector with respect to the silicon frame are fitted:

1. $x_{\text {off }}:$ the $x$-offset,

2. $y_{\text {off }}:$ the $y$-offset,

3. $z_{S}$ : the $z$-offset, i.e. the position of the shower maximum,

4. $\alpha_{\text {rot }}$ : the azimuthal rotation angle.

The results of the alignment procedure are summarized in Figure 30, which compares the measurements of the polar angle, $\vartheta$, and azimuthal angle, $\varphi$, obtained from the BGO and silicon detectors. The distributions of $\left(\vartheta_{\mathrm{sil}}-\vartheta_{\mathrm{BGO}}\right)$ and $\left(\varphi_{\mathrm{sil}}-\varphi_{\mathrm{BGO}}\right)$ vs. the azimuthal angle before the alignment are shown in Figures 30(a) and (b), respectively. The projections of these distributions onto the $\left(\vartheta_{\mathrm{sil}}-\vartheta_{\mathrm{BGO}}\right)$ and $\left(\varphi_{\mathrm{sil}}-\varphi_{\mathrm{BGO}}\right)$ axes after the global alignment are shown as the dashed histograms in Figures $30(\mathrm{c})$ and $(\mathrm{d})$.

Alignment offsets up to $3 \mathrm{~mm}$ in the transverse direction between the two detectors were found and corrected for. The same fit procedure is applied to the data as well as the Monte Carlo in order to treat them consistently. The accuracy of the fit is estimated by comparing the fit results from different time periods, as well as by comparing the fit results from the Monte Carlo with the true geometry. The precision obtained in the global alignment procedure is estimated to be $0.1 \mathrm{~mm}$ for the $x$ and $y$ offsets, $1 \mathrm{~mm}$ for the average $z$ position of the shower maximum and $1 \mathrm{mrad}$ for the rotation angle, $\alpha_{\text {rot }}$. The offsets can then be used to transform all the BGO hit coordinates back into the silicon frame. 

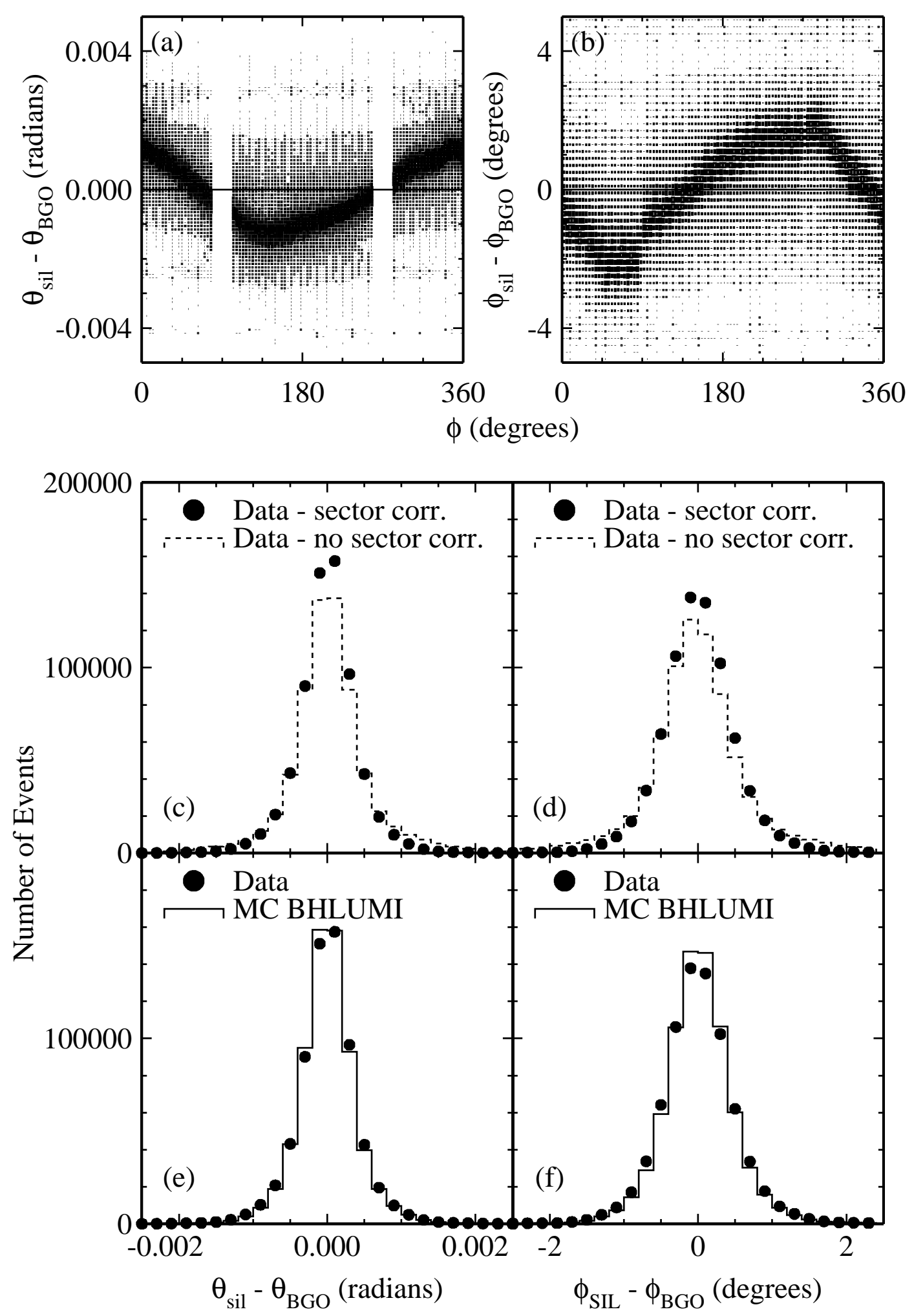

Figure 30: Matching of the BGO and silicon $\theta$ and $\phi$ measurements on the $+z$ side. (a) shows $\vartheta_{\text {sil }}-\vartheta_{\mathrm{BGO}}$ vs. $\varphi$, (b) shows $\varphi_{\text {sil }}-\varphi_{\mathrm{BGO}}$ vs. $\varphi$. (c) and (e) show the projections onto the $\vartheta_{\text {sil }}-\vartheta_{\mathrm{BGO}}$ axis before (dashed histograms) and after (points) the sector correction and a comparison with the Monte Carlo (solid histograms). (d) and (f) show the same for the projection onto the $\varphi_{\text {sil }}-\varphi_{\mathrm{BGO}}$ axis. 


\section{Sector Fit}

In a second step, the offset of each BGO sector with respect to the global fit is determined. The correction is obtained by plotting the quantities $\left(\vartheta_{\text {sil }}-\vartheta_{\mathrm{BGO}}\right)$ and $\left(\varphi_{\text {sil }}-\varphi_{\mathrm{BGO}}\right)$ for each sector. The radial and azimuthal offsets of each sector are then adjusted such that the average of each distribution is 0 . The correction has a typical magnitude of $0.1 \mathrm{~mm}$ both in the $r$ and $\varphi$ directions and never exceeds $0.5 \mathrm{~mm}(0.2 \mathrm{~mm})$ in $r(\varphi)$.

The effect of the sector corrections is visible in the reduction of the widths of the distributions in Figures 30(d) and (e). After the complete alignment, the distributions from the data and the Monte Carlo agree (shown in Figures 30(e) and (f)), demonstrating that the alignment of the detectors is well understood. ${ }^{3)}$

\subsubsection{Transverse Positioning of the Silicon}

In the next step, the silicon detector position is determined with respect to the beam axis. This alignment is also obtained from the data. The aim is to determine the offsets in $x$ and $y$ of each silicon detector with respect to the beam axis $\left(\delta_{x}, \delta_{y}\right)$ and the rotation around the $x$ and $y$ axes $\left(\alpha_{x}, \alpha_{y}\right)$. This is done using two separate fits.

The first fit to determine the offsets is based on the fact that for a perfectly aligned detector the event rates should be independent of the azimuthal angle, $\phi$. If there is an offset, the event rate as a function of the azimuthal angle in the shifted coordinate frame $\left(\phi^{\prime}\right)$ can, to first order, be parameterized as:

$$
\frac{d N}{d \phi^{\prime}}=A+B \cos \phi^{\prime}+C \sin \phi^{\prime},
$$

with the following relation between the offsets and the fit parameters:

$$
\begin{aligned}
\delta_{x} & =\frac{3 B}{8 A} z\left(\frac{1 / \theta_{\min }^{2}-1 / \theta_{\max }^{2}}{1 / \theta_{\min }^{3}-1 / \theta_{\max }^{3}}\right) \\
\delta_{y} & =\frac{3 C}{8 A} z\left(\frac{1 / \theta_{\min }^{2}-1 / \theta_{\max }^{2}}{1 / \theta_{\min }^{3}-1 / \theta_{\max }^{3}}\right),
\end{aligned}
$$

where $\theta_{\min }$ and $\theta_{\max }$ are the boundaries of the fiducial volume cuts and $z$ is the $z$ coordinate of the silicon detector.

The same fit is also applied to the fully simulated Monte Carlo events, where the offsets are found to be compatible with zero. The results of the fit to the data are shown in Table 4 . The accuracy of the fit method is found to be about $0.2 \mathrm{~mm}$, from a study using a simplified Monte Carlo that includes typical offsets and rotations.

In Figure 31 the azimuthal event density distribution is compared with the Monte Carlo before and after the correction. Before the correction, a sine-wave signal is seen in the data, indicating a detector offset. After the correction, the data and Monte Carlo agree.

The second fit to determine the rotation is made using the theta measurements from the first and second $r$-measuring detector layers. In Figure 32, the difference between these measurements is plotted versus the azimuthal angle, $\phi$. In this plot, rotations of the detector around its internal $x$ and $y$ axes show up as sine and cosine waves, respectively. This allows a very accurate determination of the rotation of the detector. The results of these fits are included in Table 4. In addition, the scatter of the points after the correction can be used as a direct check on the optical survey measurements used to determine the wafer positions. The r.m.s. of the

\footnotetext{
${ }^{3)}$ The Monte Carlo contains an ideal alignment of the BGO and silicon.
} 


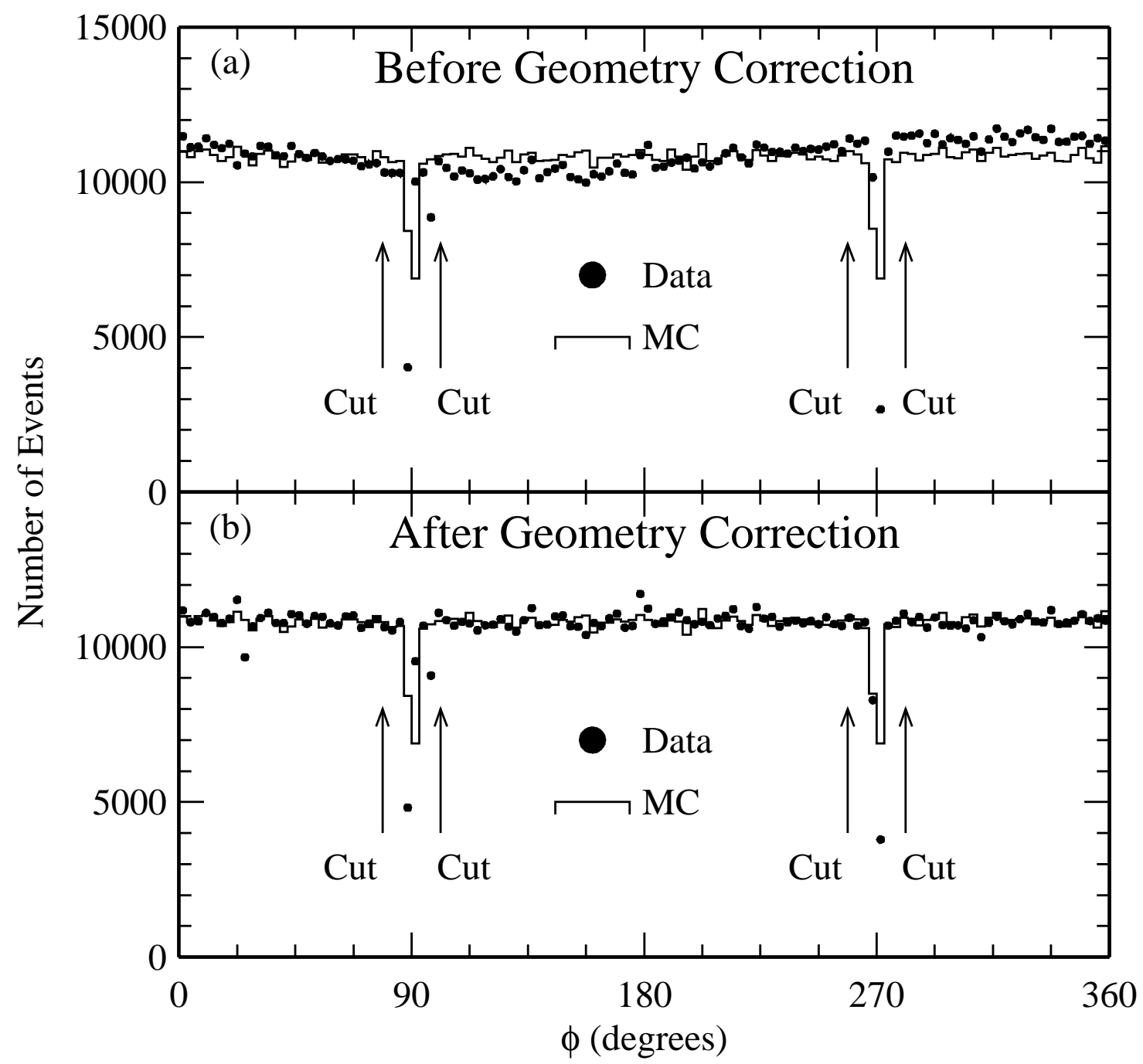

Figure 31: Azimuthal event density distribution (a) before and (b) after the geometry corrections for the data and the Monte Carlo. In the final Bhabha selection, the regions around $90^{\circ}$ and $270^{\circ}$ are removed. This is indicated with the arrows labeled as "Cut". 


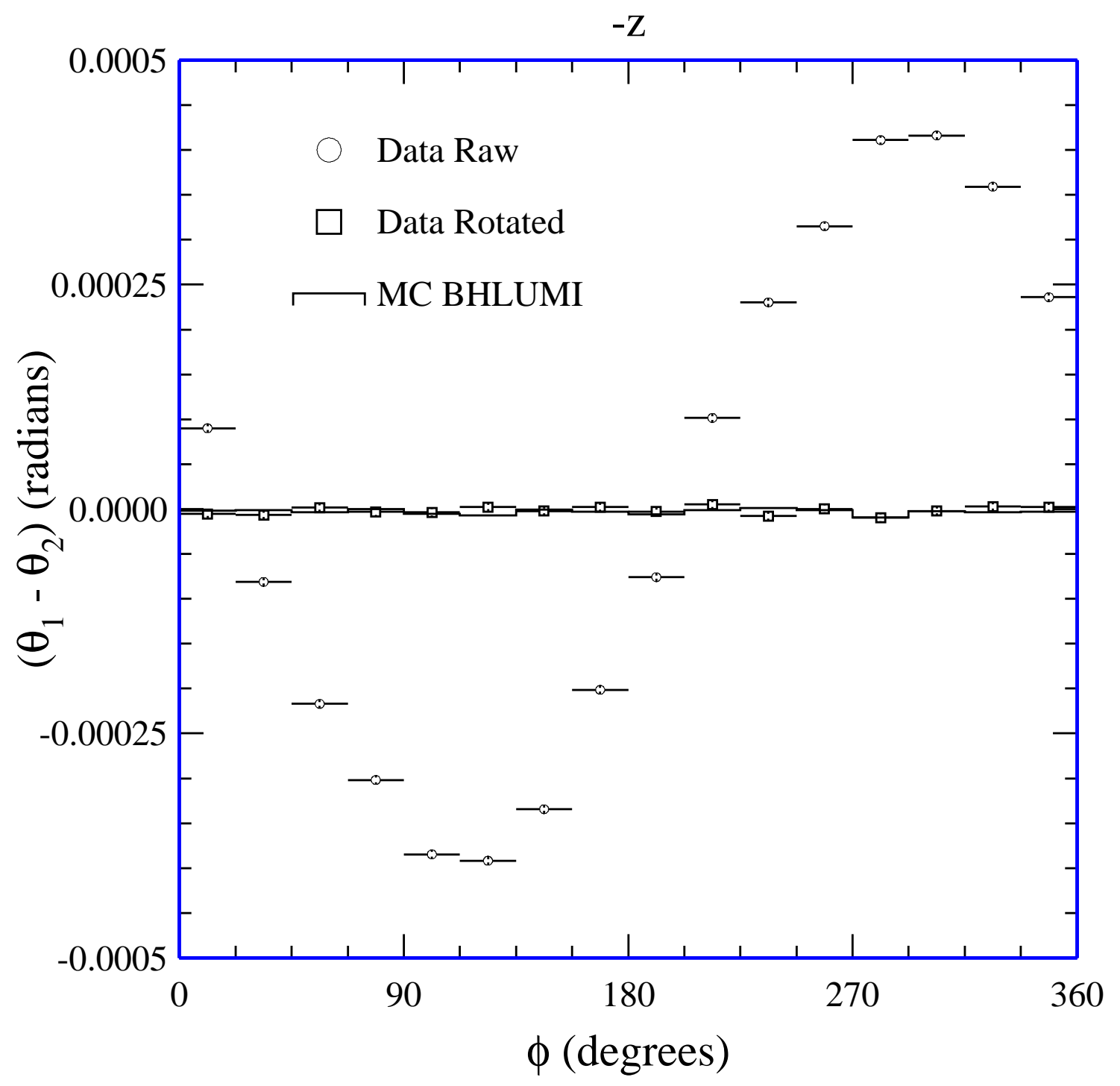

Figure 32: The difference $\left(\theta_{1}-\theta_{2}\right)$ between the $\theta$ measurements of the two $r$ silicon strip layers versus the azimuthal angle $\phi$. The data before the rotation correction (circles) and after (squares) are shown. The histogram shows the results of the Monte Carlo simulation. 


\begin{tabular}{|l|c|c|c|c|c|c|c|c|}
\hline & \multicolumn{2}{|c|}{$93 \mathrm{~A}$} & \multicolumn{2}{c|}{$93 \mathrm{~B}$} & \multicolumn{2}{c|}{$93 \mathrm{C}$} & \multicolumn{2}{c|}{94} \\
\hline & $-z$ & $+z$ & $-z$ & $+z$ & $-z$ & $+z$ & $-z$ & $+z$ \\
\hline$\delta_{x}(\mathrm{~mm})$ & -0.95 & 1.40 & -1.04 & 0.99 & -1.25 & 1.08 & 1.83 & -1.34 \\
$\delta_{y}(\mathrm{~mm})$ & $\mathbf{0 . 0 9}$ & -0.44 & 0.18 & -1.03 & 0.29 & -1.02 & -0.42 & -1.78 \\
$\alpha_{x}(\mathrm{mrad})$ & -12.5 & 8.9 & -12.2 & 8.8 & -12.0 & 3.1 & 5.2 & -4.8 \\
$\alpha_{y}(\mathrm{mrad})$ & -5.1 & -0.1 & -5.2 & -0.2 & -5.3 & -0.4 & -9.2 & -2.6 \\
\hline
\end{tabular}

Table 4: The fit of the silicon detector position for the 1993 running periods. The precision of the offsets is about $0.2 \mathrm{~mm}$ and the precision of the rotation angles is about $0.2 \mathrm{mrad}$. The change in $\alpha_{x}$, due to the change in the external cabling of the $+z$ detector between periods $93 \mathrm{~B}$ and $93 \mathrm{C}$, is clearly seen.

$\left(\theta_{1}-\theta_{2}\right)$ distribution corresponds to a scatter in the wafer positions of $11 \mu \mathrm{m}$. The corresponding number in the Monte Carlo is $8 \mu \mathrm{m}$. Assuming that the difference between the data and the Monte Carlo is due to the uncertainty in the wafer position, an extra scatter of $7 \mu \mathrm{m}$ on the wafer position for the data is obtained. This corresponds to an additional uncertainty of $2 \mu \mathrm{m}$ on the average radius of each layer, which is small compared to the overall $6 \mu \mathrm{m}$ uncertainty on the detector geometry.

\subsubsection{Longitudinal Positioning of the Silicon}

The final luminosity is obtained by averaging the event rates observed within the fiducial volume at both sides of the interaction point, This cancels to first order the effects of a displacement of the interaction point with respect to the center of the detector. To obtain the best possible luminosity measurement for each detector separately, the distance of each detector with respect to the vertex must be determined. The average position of the interaction point is measured using the collinearity of the Bhabha events. This position can be compared with the geometric center of the L3 experiment, assuming that the silicon trackers are at the positions determined from survey. The offset of the interaction point from the center was $-0.2 \mathrm{~mm}$ in 1993 and $-0.1 \mathrm{~mm}$ in 1994 . The error on this position is dominated by systematics and has been estimated to be $0.3 \mathrm{~mm}$ by fitting the Monte Carlo using the same method and comparing different ways of fitting the data. The values are close to zero, showing that the beams collide near the center of the experiment. 


\section{Luminosity}

Bhabha events are triggered using the luminosity, single-tag and double-tag triggers (Section 2.2). The events used in the luminosity calculation are required to have passed the double-tag trigger. Bhabha events are selected using the calorimetric measurement in the BGO to provide a background free sample of events, and the silicon tracker to select only those Bhabhas that are contained in a precisely defined fiducial volume.

\section{$5.1 \quad$ Event Selection}

A sample Bhabha event is shown in Figure 33. The plot shows the outline of the BGO crystals in grey and the borders of the silicon detector in black. The energy depositions in the BGO crystals are presented as black squares, with an area proportional to the energy deposition. The line segments indicate the silicon strips which contained a reading above threshold for this event. The line thickness indicates the size of the silicon strip. Ideally, a single Bhabha electron causes a hit in three silicon strips and deposits its energy in approximately 25 BGO crystals. With a minimum energy cut of $200 \mathrm{MeV}$ on the energy in a crystal, on average 12 crystals are used in the fit to the shower shape (Section 2.3), which yields the energy of the particle and its point of impact in the detector. The energy requirements for an event to be classified as a Bhabha are:

- The reconstructed energy of the most energetic cluster on one side should be $E>$ $0.8 E_{\text {beam }}$.

- The reconstructed energy of the most energetic cluster on the opposite detector side should be $E>0.4 E_{\text {beam }}$.

- If the most energetic cluster on one side has $E>0.95 E_{\text {beam }}$, the minimum energy requirement on the other side is reduced to $0.2 E_{\text {beam }}$.

The asymmetric energy cut means that most radiative Bhabha events are accepted and minimizes the effect of any malfunctioning crystals. The last cut selects a small fraction $(\approx 0.1 \%)$ of events observed in the 1994 data, where the electron or positron passes between the crystals and only deposits a small amount of energy in the calorimeter. The number of such events depends on the orientation of the detector with respect to the beam and can therefore vary from year to year.

In addition, a Bhabha event must pass an acoplanarity requirement:

- The difference, $\Delta \phi$, between the azimuthal angles of the two particles must satisfy: $\left|\Delta \phi-180^{\circ}\right|<10^{\circ}$.

The selection of the Bhabha events inside the fiducial volume relies on the coordinate reconstruction, mainly using the hits in the silicon tracker. The fiducial volume is defined by asymmetric requirements (with respect to the detectors) in order to be insensitive to offsets of the detectors with respect to the beam axis. In practice, this means that a Bhabha is required to pass a tight cut on one side of the detector and, simultaneously, a loose cut on the opposite side. The fiducial volume for the Bhabha sample is chosen such that each selected Bhabha event is fully contained in the detector in order to avoid edge effects in the reconstruction. The requirements are: 


\section{Bhabha Event in the L3 Luminosity Monitor}
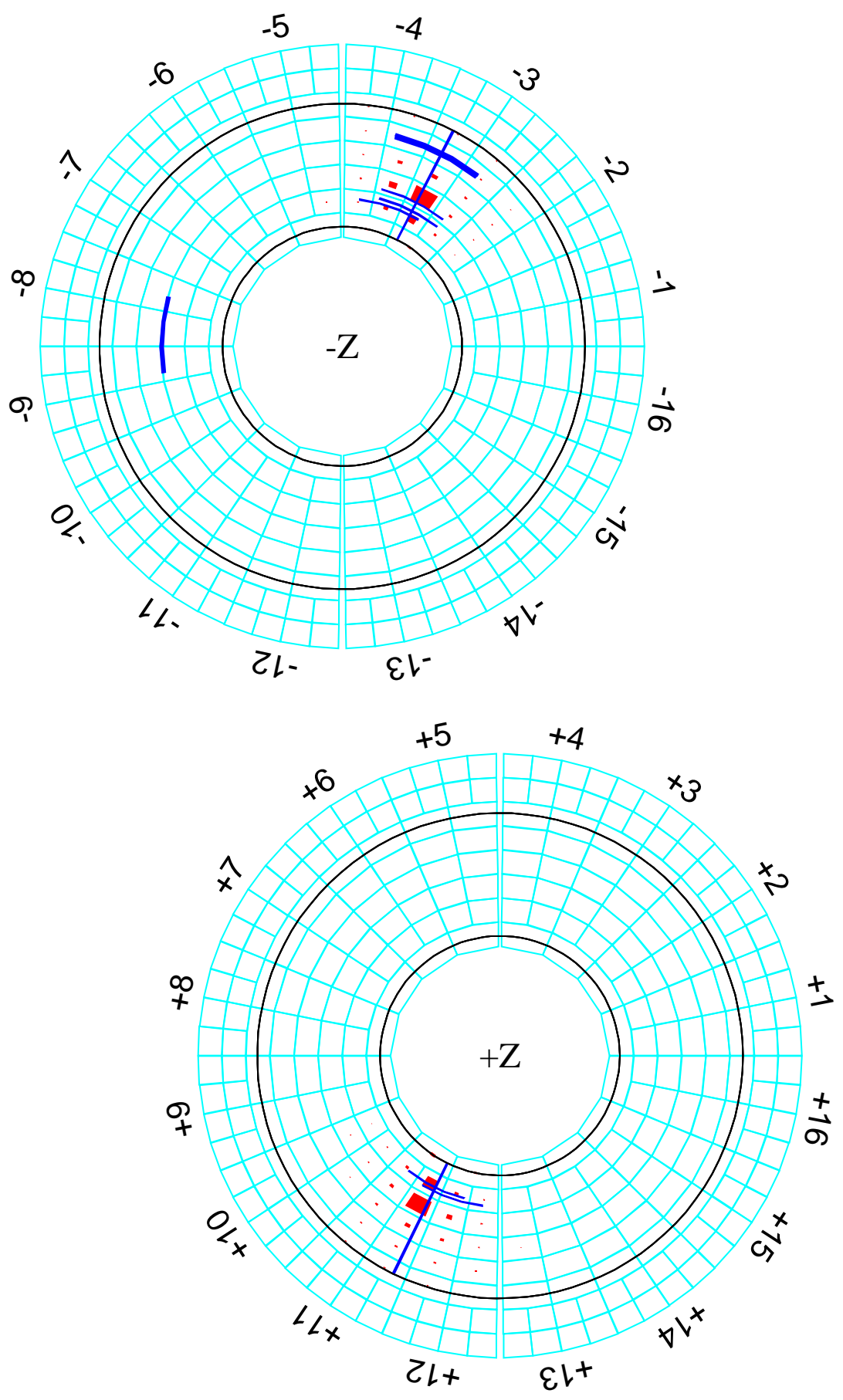

Figure 33: A Bhabha event in the luminosity monitor. The BGO crystals are displayed in grey and the circular outline of the silicon detector in black. The energy detected in the BGO is shown by squares, the areas of which are proportional to the amount of energy deposited. The hits in the silicon detector are shown by highlighted line segments. Note that the silicon strips and BGO crystals are shown in cylindrical coordinates. Since they are located at different $z$ positions, the silicon hit occurs at a somewhat smaller radius than the BGO impact point. 
- Tight: The polar angle must be in the domain: $32<\theta<54$ mrad.

The azimuthal angle to be in the domain: $\left|\phi-90^{\circ}\right|>11.25^{\circ}$ and $\left|\phi-270^{\circ}\right|>11.25^{\circ}$.

- Loose: The polar angle must be in the domain: $27<\theta<65$ mrad.

The azimuthal angle must be in the domain: $\left|\phi-90^{\circ}\right|>3.75^{\circ}$ and $\left|\phi-270^{\circ}\right|>3.75^{\circ}$.

The cuts on the azimuthal angle ensure that the selected events are away from the vertical plane, because this region is insensitive due to the split in the BGO calorimeter.

The resulting energy distribution for Bhabha events is displayed in Figure 34. The distribu-

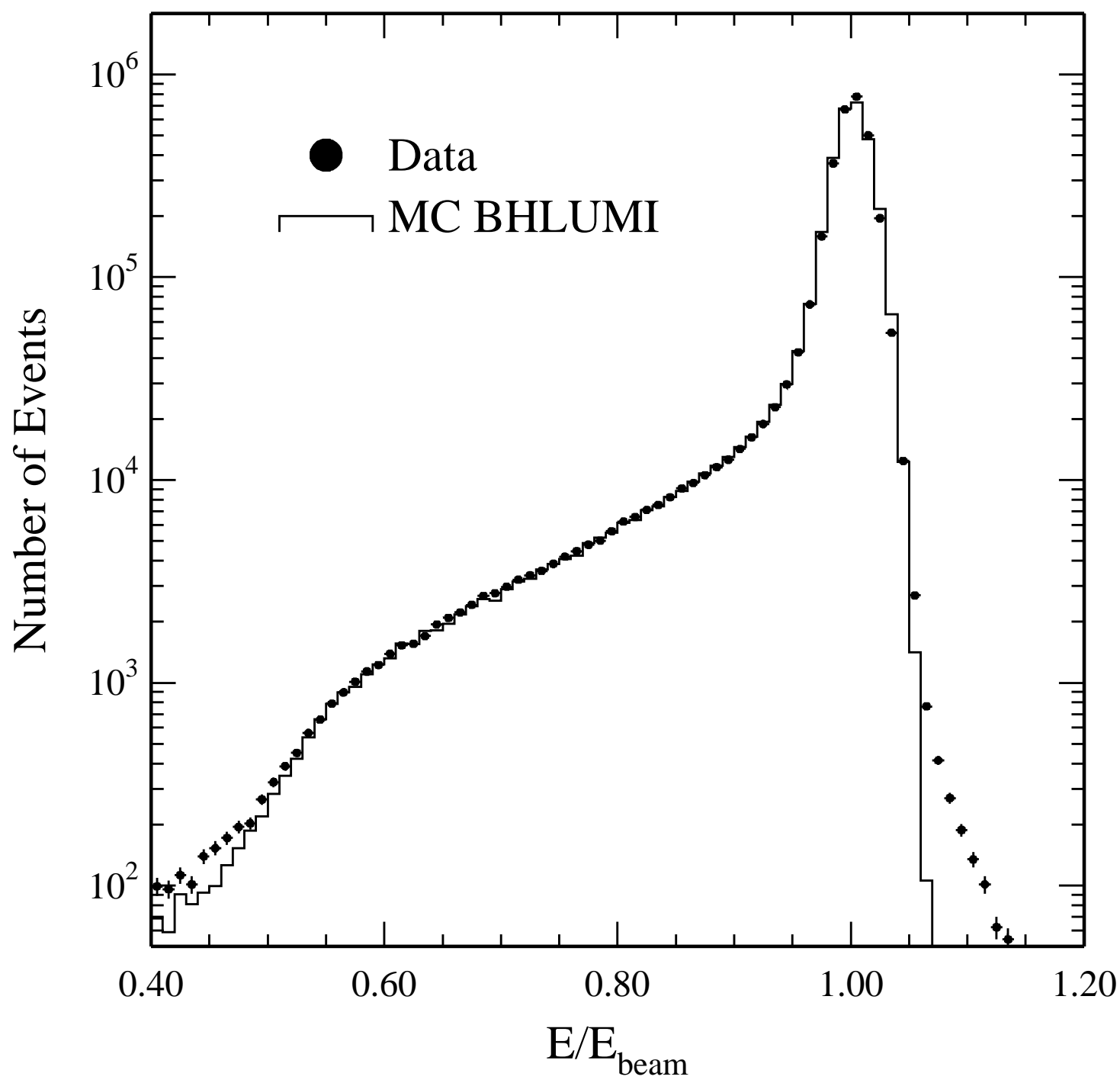

Figure 34: Observed energy of the Bhabha electrons divided by the beam energy for 1993. The circles correspond to the data, the histogram to the Monte Carlo. The slight excess at high energies in the data is due to the overlap of a Bhabha event with a shower from a beam gas interaction.

tions for the acoplanarity and for the polar angle are shown in Figures 35 and 36, respectively, where they are also compared with the Monte Carlo. All the alignment corrections described in the previous section have been applied. The data and Monte Carlo are normalized to the same area. 
The theta distribution is shown both for the $-z$ and $+z$ sides. The $-z$ distribution shows an enhancement of events at the low theta edge. This effect is due to Bhabha events at even lower polar angles in which the particles scatter from the beam pipe into the fiducial volume. The effect is reduced on the $+z$ side, where the beam pipe is effectively thinner. The effect of the flared part of the beam pipe is seen on the $+z$ side in the region between $42 \mathrm{mrad}$ and $52 \mathrm{mrad}$. Here, the otherwise smooth distribution is somewhat distorted due to the presence of one radiation length of material in front of the detector. However, this distortion causes no harm as the fiducial volume cuts are applied in the smooth part of the distribution. The tight fiducial volume cuts are indicated in the plot.

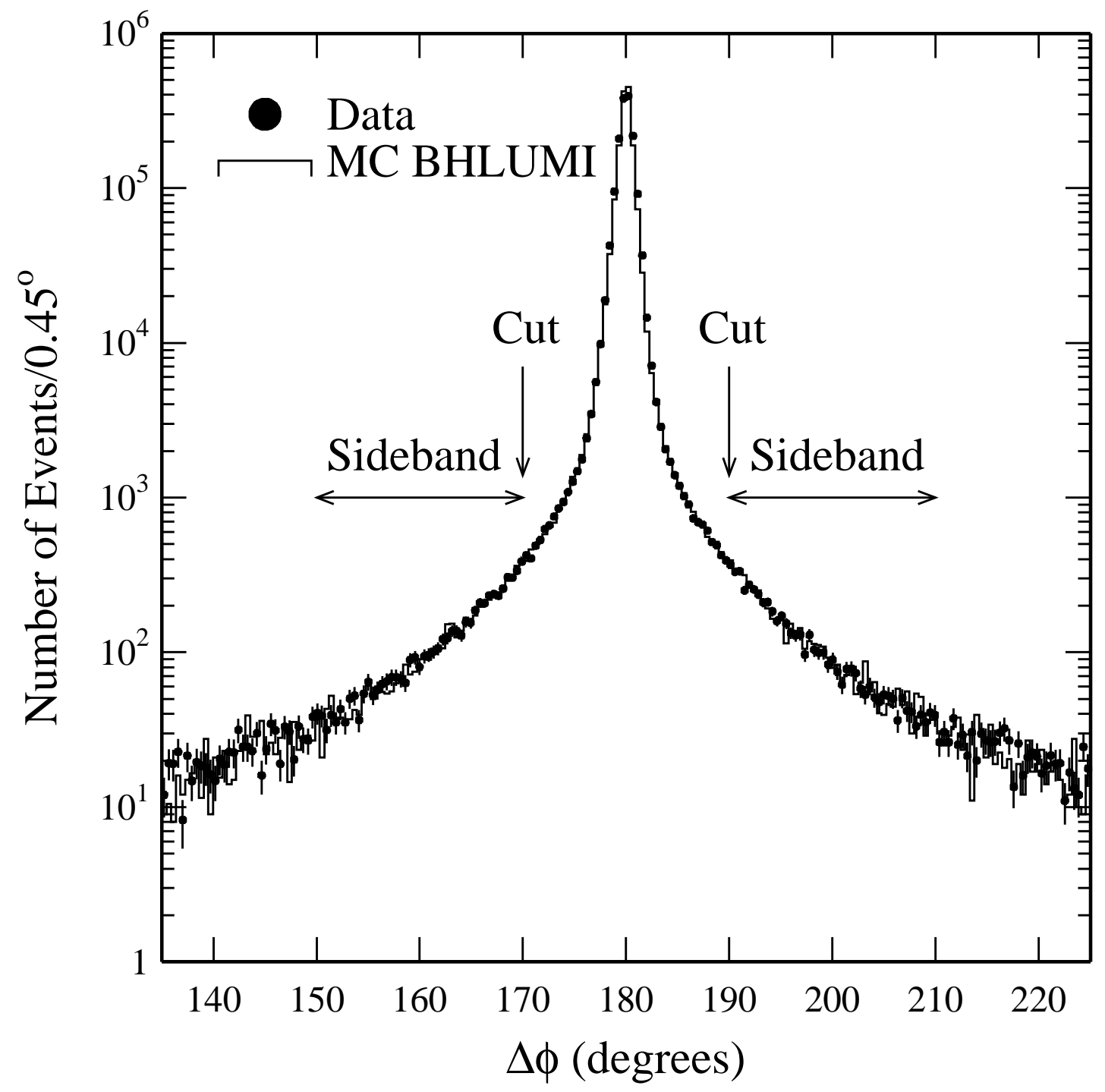

Figure 35: The acoplanarity distribution of the Bhabha events. The circles indicate the data and the histogram is the BHLUMI Monte Carlo result. The position of the cut is indicated by the vertical arrows and the sideband region, which is used for background subtraction, by the horizontal arrows. 


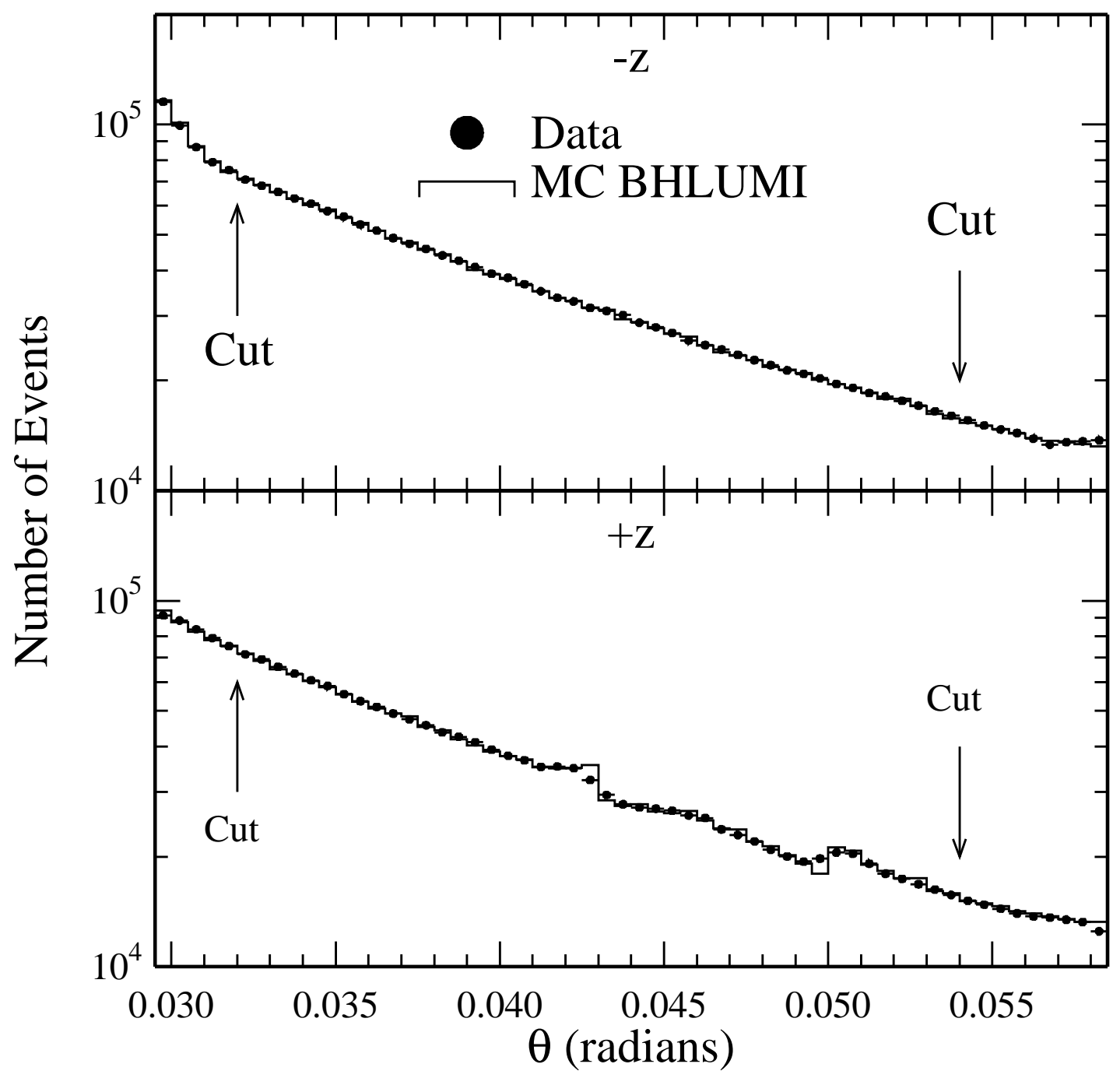

Figure 36: The polar angle distribution of the reconstructed Bhabha events for the $-z$ and $+z$ sides from the data and the Monte Carlo. The position of the tight fiducial volume cut is indicated by the arrows. 


\subsection{Visible Cross Section}

To determine the visible cross section for Bhabha scattering, $\mathrm{e}^{+} \mathbf{e}^{-} \rightarrow \mathrm{e}^{+} \mathbf{e}^{-}(\gamma)$, events are generated at $\sqrt{s_{0}}=91.25 \mathrm{GeV}$ using the BHLUMI V2.01 Monte Carlo program [2]. At the generator level, the momentum transfer variable, $t$, is required to be in the range $0.83<t<$ $83.0 \mathrm{GeV}^{2}$, which corresponds to a polar angular range of approximately $0.020<\theta<0.200 \mathrm{rad}$ for the scattered electron and positron, comfortably including the complete coverage of the detector. The generated events are passed through the L3 simulation program [4]. The dead crystals for each year of detector operation are also killed in the reconstruction of the Monte Carlo events.

As the event selection does not differentiate between electrons and photons, a contribution from the $\mathrm{e}^{+} \mathrm{e}^{-} \rightarrow \gamma \gamma(\gamma)$ process $(0.02 \%)$ must be added to the visible cross section. The small background from the double-tag mode of the two-photon process, $\mathrm{e}^{+} \mathrm{e}^{-} \rightarrow \mathrm{e}^{+} \mathrm{e}^{-} X$, is generally not coplanar and is therefore accounted for by the $\Delta \phi$ sideband background subtraction procedure described below. The visible cross section is based on a sample of 11.2 million generated and fully simulated events. The systematic uncertainty in the visible cross section due to the Monte Carlo statistics is $\mathbf{0 . 0 6 \%}$. The theoretical uncertainty due to the approximations used in the BHLUMI V2.01 calculation is estimated to be $0.25 \%$ [2]. A new version of BHLUMI, V4.03, has an improved theoretical precision of $0.11 \%$ [3]. The difference between the accepted cross sections for the two versions of BHLUMI, determined using a generator-level simulation of the detector, is negligible: $(0.02 \pm 0.03) \%$. An uncertainty of $0.11 \%$ is therefore assigned as the estimate of the systematic uncertainty due to theory.

The energy dependence of the $t$-channel contribution to the cross section is also estimated using BHLUMI V4.03. This dependence can be parameterized by $\left(s_{0} / s\right)^{0.9933}$, i.e. slightly smaller than the lowest-order $\left(s_{0} / s\right)$ expectation.

The contribution of the Z-exchange diagram in BHLUMI V2.01 is a zeroth-order calculation. For the main contribution, the $\gamma$-Z interference, an $\mathcal{O}(\alpha)$ calculation is necessary for a high precision luminosity measurement. An exponentiated version of this calculation has recently been included in BHLUMI V4.03 [3]. The Monte Carlo generator BABAMC [14] contains an $\mathcal{O}(\alpha)$ prediction without exponentiation. Since the Bhabha Monte Carlo detector simulation has been run using BHLUMI V2.01 at $\sqrt{s}=91.25 \mathrm{GeV}$ with the zeroth-order Z-exchange contribution included, the $\mathrm{Z}$ effect can either be removed from BHLUMI V2.01 and replaced with the more accurate determination from BABAMC or be evaluated directly with BHLUMI V4.03. The fitted $\mathrm{Z}$ contribution as a function of $\sqrt{s}$, evaluated using the two methods, is shown in Figure 37. The systematic error on the BABAMC contribution is estimated to be $\mathbf{0 . 0 3 \%}$, which is the same as the result found in Reference [15].

In Figure 37 it can be seen that the $\mathrm{Z}$ contribution determined using the two methods is in good agreement for center-of-mass energies below the $\mathrm{Z}$ whereas there is a small, but significant difference above the peak, which is due to the photon exponentiation. For the final visible cross section we therefore use the $\mathrm{Z}$ interference term calculated using BHLUMI V4.03 and assign a systematic error of $0.015 \%$ [3] for the uncertainty.

At $\sqrt{s}=91.25 \mathrm{GeV}$ the visible cross section including all corrections is $69.62 \pm 0.04 \mathrm{nb}$.

\subsection{Background}

The number of background events in the final sample is estimated using the sidebands of the acoplanarity distribution. An attempt is made to select background events by demanding that these events have an energy of $E_{1}<0.95 E_{\text {beam }}$ and $E_{2}<0.95 E_{\text {beam. }}$ In addition, the event 


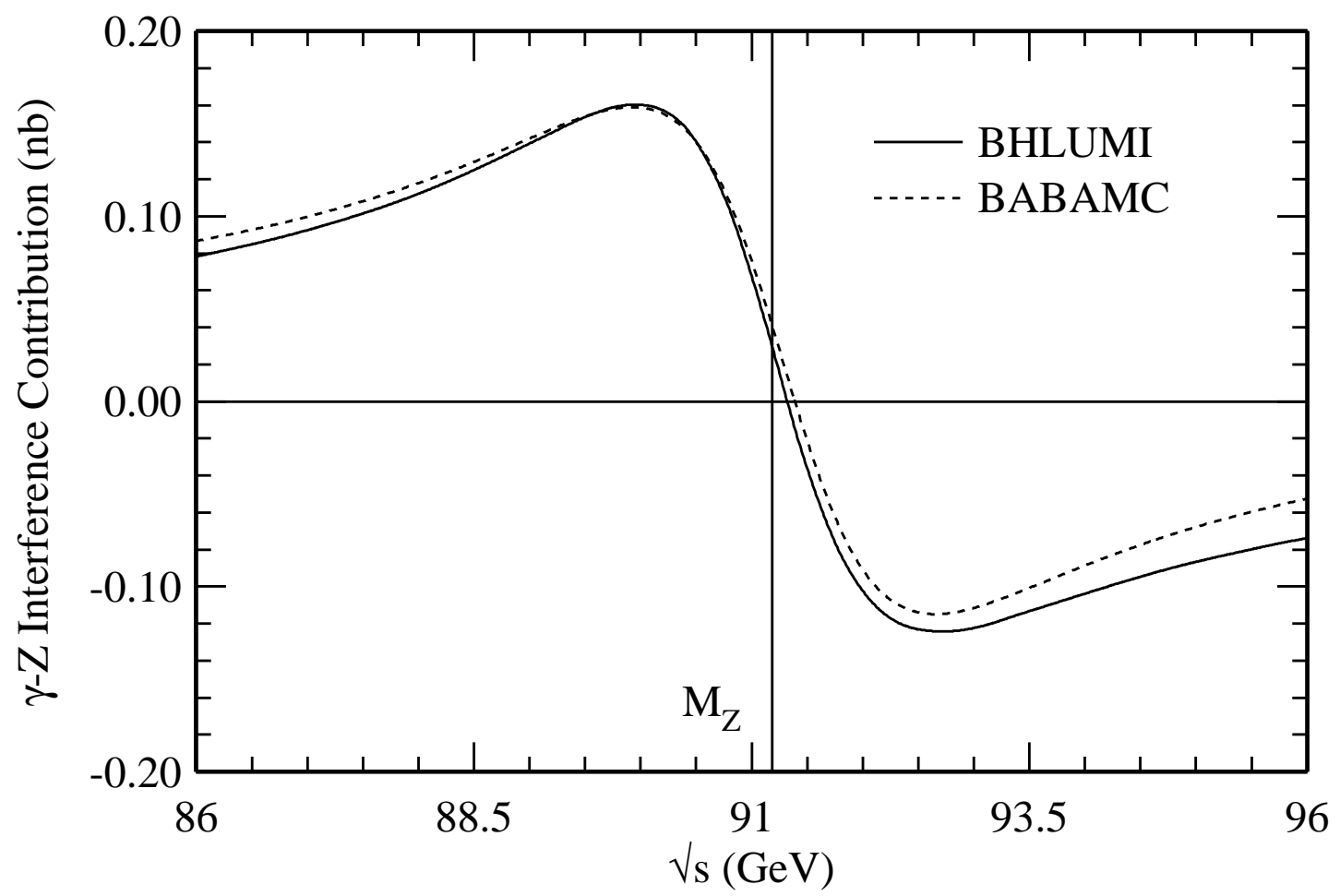

Figure 37: The $\mathrm{Z}$ contribution to the small-angle Bhabha cross section, as calculated using BHLUMI V4.03 and BABAMC in the region of the $\mathrm{Z}$ pole.

should have an acoplanarity of $10^{\circ}<\left|\Delta \phi-180^{\circ}\right|<30^{\circ}$. The background present in the final Bhabha sample is then estimated by assuming that the background distribution is flat in the acoplanarity variable $\Delta \phi$. The number of selected background events in the sidebands are extrapolated to the area under the peak, $\left|\Delta \phi-180^{\circ}\right|<10^{\circ}$. The same algorithm is applied to the data $\left(N_{\text {back }}^{\text {Data }} / N_{\text {Bhabha }}^{\text {Data }}=(2.87 \pm 0.12) \cdot 10^{-4}\right)$ and to the Monte Carlo, $\left(N_{\text {back }}^{\mathrm{MC}} / N_{\text {Bhabha }}^{\mathrm{MC}}=\right.$ $\left.(2.53 \pm 0.18) \cdot 10^{-4}\right)$ and the difference is assigned to be the background, $(0.34 \pm 0.22) \cdot 10^{-4}$.

\subsection{Luminosity Determination}

The integrated luminosity is calculated from:

$$
\mathcal{L}=\frac{1}{\varepsilon} \frac{N_{\text {acc }}}{\sigma^{\text {vis }}}
$$

in which $N_{\text {acc }}$ is the accepted number of Bhabha events inside the fiducial volume, $\sigma^{\text {vis }}$ is the visible cross section for Bhabha scattering in the same fiducial volume and $\varepsilon$ accounts for any inefficiency in the data that is not included in the Monte Carlo, e.g. the trigger. Since the silicon detector has two theta-measuring planes on either side of the interaction point, the number of accepted Bhabha events can be measured four times. Based on the information from each of these four layers the event selection criteria are applied as described in Section 5.1.

For each layer, $i$, an event, $j$, is given a weight, $W_{i}^{j}$, of 1 if it passes the energy and acoplanarity cuts and is inside the tight fiducial volume for layer number $i$ and inside the loose fiducial volume cut on the other side. Otherwise it is given a weight of 0 for that layer. The number of Bhabha events per layer, $N_{i}^{\text {Data }}$, can be defined as the sum of the event weights over 
all triggers, $N_{\text {trig: }}$ :

$$
\begin{aligned}
N_{i}^{\text {Data }} & =\sum_{j=1}^{N_{\text {trig }}} W_{i}^{j} \\
N_{w}^{\text {Data }} & =\frac{1}{4}\left(N_{1}^{\text {Data }}+N_{2}^{\text {Data }}+N_{3}^{\text {Data }}+N_{4}^{\text {Data }}\right) .
\end{aligned}
$$

Similarly, in the case of simulated data, the sum runs over all generated events, $N_{\text {gen }}^{\mathrm{MC}}$ :

$$
\begin{aligned}
N_{i}^{\mathrm{MC}} & =\sum_{j=1}^{N_{\text {gen }}^{\mathrm{MC}}} W_{i}^{j} \\
N_{w}^{\mathrm{MC}} & =\frac{1}{4}\left(N_{1}^{\mathrm{MC}}+N_{2}^{\mathrm{MC}}+N_{3}^{\mathrm{MC}}+N_{4}^{\mathrm{MC}}\right) .
\end{aligned}
$$

During the 1993 run, $7.6 \times 10^{6}$ triggers were recorded, yielding $N_{w}^{\text {Data }}=2.3 \times 10^{6}$ accepted Bhabhas. For 1994 the numbers are $9.8 \times 10^{6}$ and $N_{w}^{\text {Data }}=3.5 \times 10^{6}$ respectively. The number of simulated BHLUMI V2.01 events, $N_{\text {gen }}^{\mathrm{MC}}$, was $11.2 \times 10^{6}$, out of which $N_{w}^{\mathrm{MC}}=2.4 \times 10^{6}$ were accepted.

Using the above definitions, the luminosity corresponding to a tight fiducial volume in layer $i$ is defined as:

$$
\mathcal{L}_{i}=\frac{1}{\varepsilon} \frac{N_{i}^{\text {Data }}}{\sigma_{i}^{\text {vis }}}=\frac{1}{\varepsilon} \frac{N_{i}^{\text {Data }}}{N_{i}^{\mathrm{MC}}} \frac{N_{\text {gen }}^{\mathrm{MC}}}{\sigma_{\text {gen }}^{\mathrm{MC}}} f_{s_{0} / s}(s) f_{\gamma \gamma} f_{\gamma \mathrm{Z}}(s),
$$

where $\sigma_{\text {gen }}^{\mathrm{MC}}$ indicates the Bhabha cross section obtained with BHLUMI V2.01, $f_{s_{o} / s}(s)$ is the center-of-mass energy correction, $f_{\gamma \gamma}$ is the correction for $\mathrm{e}^{+} \mathrm{e}^{-} \rightarrow \gamma \gamma(\gamma)$ and $f_{\gamma \mathrm{Z}}(s)$ is the $\gamma-\mathrm{Z}$ interference correction. The final integrated luminosity is then calculated from:

$$
\mathcal{L}_{w}=\frac{1}{\varepsilon} \frac{N_{w}^{\text {Data }}}{\sigma_{w}^{\text {vis }}}=\frac{1}{\varepsilon} \frac{N_{w}^{\text {Data }}}{N_{w}^{\mathrm{MC}}} \frac{N_{\text {gen }}^{\mathrm{MC}}}{\sigma_{\text {gen }}^{\mathrm{MC}}} f_{s_{0} / s}(s) f_{\gamma \gamma} f_{\gamma \mathrm{Z}}(s) .
$$

The quantities $\mathcal{L}_{i}$ are a separate integrated luminosity measurement for each silicon detector $r$ layer (used for the systematic error study). Note that $\mathcal{L}_{w}$ is not necessarily equal to $1 / 4 \sum \mathcal{L}_{i}$. However, numerically they agree within 1 part in $10^{6}$.

\subsection{Radiative Bhabha Events}

Radiative effects are important for a precise measurement of the luminosity. There is a relatively large contribution to the systematic uncertainty due to incomplete modeling of higher order effects in the Monte Carlo event generators for Bhabha scattering. There is also an interplay between the Bhabha event selection criteria on one hand and the combination of photon radiation and an imperfect detector geometry on the other, because radiative events are not necessarily collinear and therefore offsets of the beam with respect to the detector no longer cancel.

To investigate the modeling of radiative effects, two very different topologies of radiative Bhabha events are selected:

1. Radiative Bhabha events where, in addition to the scattered electron or positron, there is a photon in one of the BGO calorimeters. These are labeled as "final-state" photon events. 
2. Radiative Bhabha events where, in addition to the scattered electron and positron, a photon escapes along the beam direction. These are labeled as "initial-state" photon events.

Theoretically, photons from initial and final-state radiation are indistinguishable; the names "initial-state" and "final-state" are only used to identify the two selected event samples. Typical examples of such events are shown in Figure 38.

The event selection and the comparison of real and simulated data for these two event samples are discussed below. Both samples are obtained by the addition of selection criteria to a slight modification of the standard Bhabha event selection cuts discussed in Section 5.1. Particles are identified with a cluster in the BGO calorimeter; the kinematic variables $(E, \theta, \phi)$ of these clusters are defined as in Section 2.3 including the use of SLUM coordinates as defined in Section 3.11. The differences from the standard Bhabha event selection are:

(a) The fiducial volume ranges from $32-55 \mathrm{mrad}$ and is the same for both sides of the interaction point, i.e. no tight-loose selection. The initial-state events are not collinear, and therefore the loose fiducial volume cut has much more effect on the event selection.

(b) For the final-state radiative events, the kinematic variables of two particles reconstructed within a single BGO calorimeter are combined into a single "particle" via an energyweighted vector addition.

\subsubsection{Monte Carlo Predictions}

For the study of radiative Bhabha scattering, the BHLUMI V2.01 event generator is used. At the generator level a Bhabha event sample is selected using the selection criteria described in the previous section. In this sample, the energies and momenta of all generated photons are summed to yield the total photon energy and momentum. Events with a total photon energy of less than $0.05 E_{\text {beam }}$ are ignored. Subsequently, the polar angle of the total photon momentum is used to characterize the selected event: initial-state radiation if the polar angle is below $20 \mathrm{mrad}$, and final-state radiation if the polar angle is in the range $32-55 \mathrm{mrad}$.

Figure 39 shows the $\mathrm{e}^{ \pm}$energy distribution for all selected Bhabha events, as well as for the samples selected as initial and final-state radiation. For the L3 detector geometry the initial and final-state radiation samples amount to about $10 \%$ and $1.5 \%$ of the selected Bhabha event sample, respectively. This provides a quantitative estimate of the relative importance of initial and final-state radiation effects. It also explains why care is required to avoid biases in the Bhabha event selection due to initial-state radiation effects.

\subsubsection{Final-State Radiation}

A typical $\mathbf{e}^{+} \mathbf{e}^{-} \rightarrow \mathbf{e}^{+} \mathbf{e}^{-} \gamma$ event is shown in Figure 38(a). Three distinct electromagnetic showers can be identified in the BGO calorimeter. The BGO calorimeters alone do not allow a distinction between a $\gamma$ and an $\mathrm{e}^{ \pm}$, and there is both too much material and too much variation in the amount of material in front of the silicon tracker to make a reliable separation between charged and neutral particles. Therefore, the photon is identified as the least energetic of the two showers in a calorimeter. Events with more than two clusters on one side are rejected. Some of these events have two final-state photons, a small fraction are two Bhabha events from the same bunch crossing and the others are final-state radiation with beam-gas contamination. Since these categories are hard to separate and the statistics are too low to make a detailed 

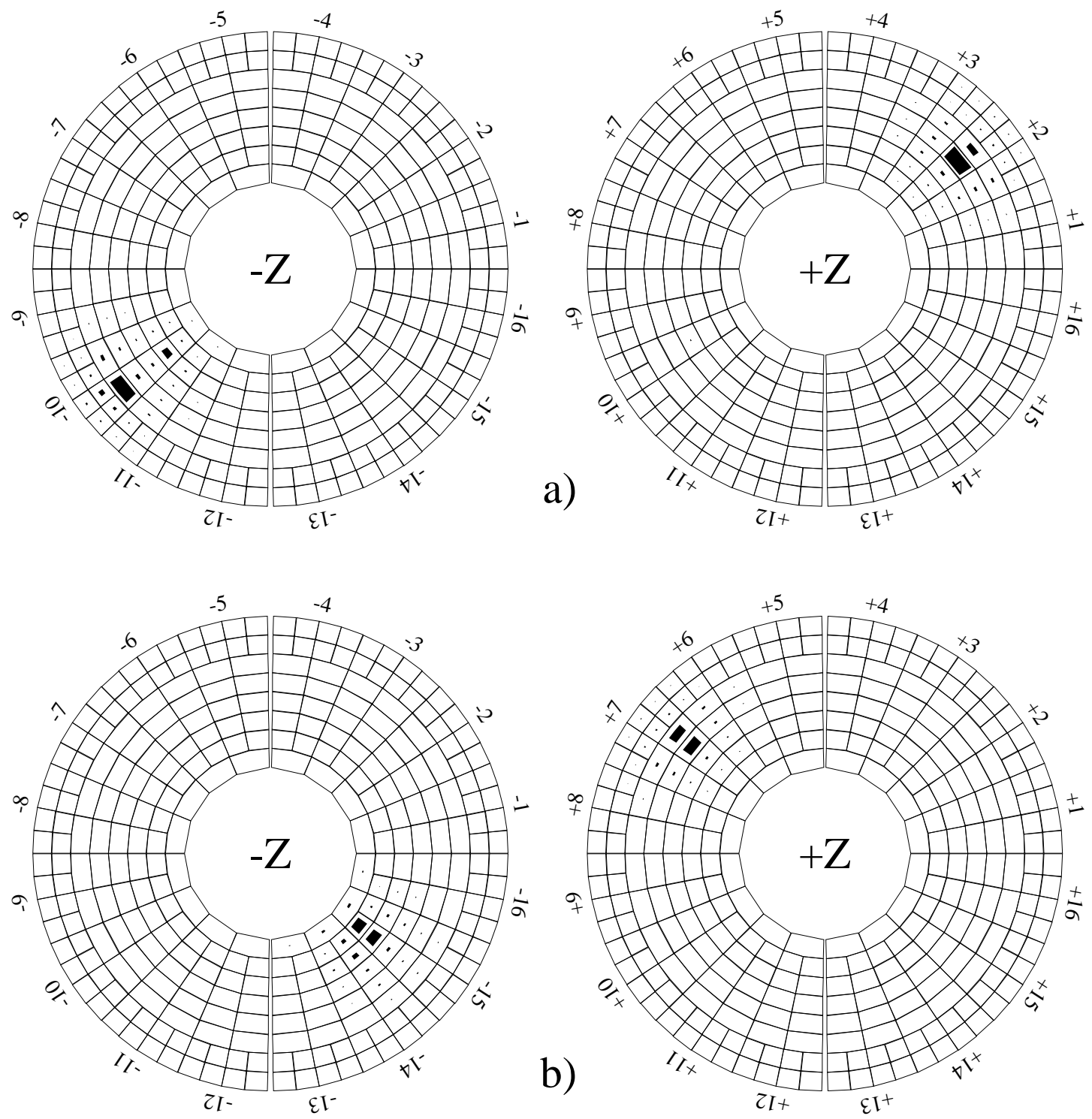

Figure 38: (a) Example of an $\mathbf{e}^{+} \mathbf{e}^{-} \rightarrow \mathrm{e}^{+} \mathrm{e}^{-} \gamma$ ("final-state") event with all three final-state particles detected in the luminosity monitor. The 2 particles in sector -10 are identified with the clusters centered in rings 3 and 6 . (b) Example of an $\mathbf{e}^{+} \mathbf{e}^{-} \rightarrow \mathbf{e}^{+} \mathbf{e}^{-}(\gamma)$ ("initial-state") event. In this case, the presence of a photon is deduced from the lack of energy-momentum balance. 


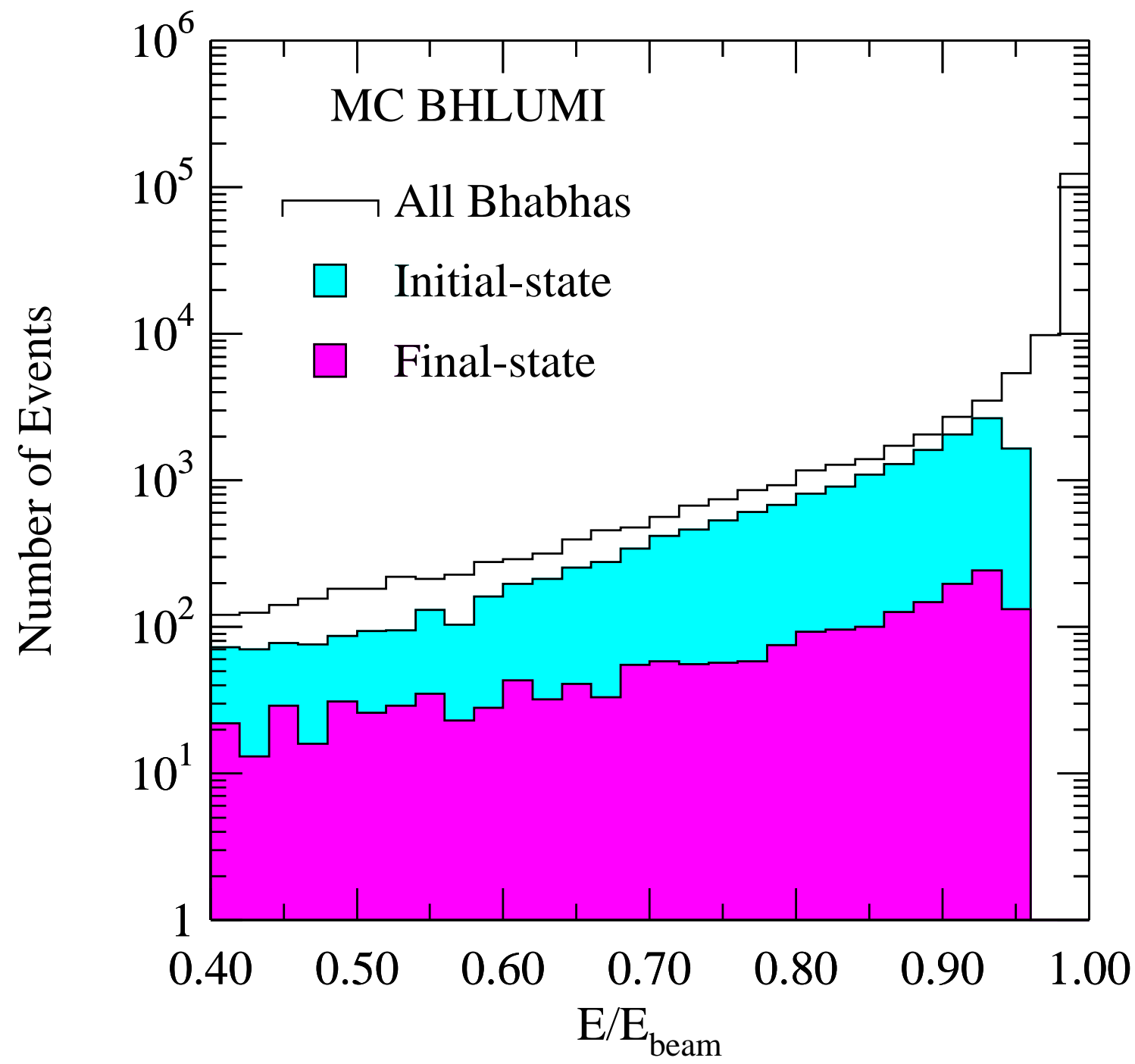

Figure 39: $\mathrm{e}^{ \pm}$energy distribution for all Monte Carlo Bhabha events. The initialstate radiation and final-state radiation events are indicated separately. 
comparison between the data and the Monte Carlo, no correction to the cross section is applied. The effect is estimated to be less than $1 \%$ of the final-state radiation cross section. The effect on the luminosity measurement is negligible since most of these events pass the standard Bhabha selection criteria.

Only photons with an energy exceeding $0.10 E_{\text {beam }}$ are retained. For a genuine radiative Bhabha event, the sum of the photon energy, $E_{\gamma}$, and the energy of the electron (or positron), $E_{\mathrm{e}}$, must be close to the beam energy. To suppress the potentially large background due to the superposition of a beam-gas interaction and a non-radiative Bhabha event, all events with $\left|E_{\gamma}+E_{\mathrm{e}}-E_{\text {beam }}\right|>0.10 E_{\text {beam }}$ are rejected. The effect of this requirement can be seen in Figure 40(a). This is the only additional requirement used to tag final-state radiation.

In Figure 40(b), the measured differential cross section for final-state radiation is shown as a function of the photon energy. The same figure also shows the BHLUMI Monte Carlo prediction. The cutoff close to $E_{\gamma}=0.5 E_{\text {beam }}$ is due to the photon identification requirement. The data and simulation are in excellent agreement.

The ratio of the integrated cross sections above $E_{\gamma}=0.10 E_{\text {beam }}$ is

$$
\frac{\int d \sigma / d E_{\gamma}(\text { Data }) d E_{\gamma}}{\int d \sigma / d E_{\gamma}(\mathrm{MC}) d E_{\gamma}}=0.993 \pm 0.010 \pm \mathbf{0 . 0 1 3},
$$

where the first uncertainty is due to statistics, mostly from the Monte Carlo simulation, and the second uncertainty is due to systematics. The systematic error is estimated using the assumption that the overall systematic error on the energy scale is $0.1 \%$, as discussed in Section 2.4 . No systematic deviation of the data with respect to the Monte Carlo is observed.

\subsubsection{Initial-State Radiation}

A typical $\mathbf{e}^{+} \mathbf{e}^{-} \rightarrow \mathbf{e}^{+} \mathbf{e}^{-}(\gamma)$ candidate event, where the photon escapes along the beam line, is shown in Figure 38(b). Such events are characterized by the presence of two energy deposits in the BGO calorimeters: one with an energy close to the beam energy and one on the opposite side of the interaction point with an energy substantially smaller than $E_{\text {beam. }}$. Events with more than one cluster on one side are rejected. The sample of events with more than one cluster on one side consists mostly of events with both an initial-state and a final-state photon. However, slightly more events of this type are selected in the data than in the Monte Carlo. This enhancement is assumed to be caused by initial-state events contaminated with a beam-gas interaction. The difference between the data and Monte Carlo (1\%) is used as a correction.

Conservation of transverse momentum imposes the following relationship between the measured energies, $E_{ \pm}$, and polar angles, $\theta_{ \pm}$, of the two clusters:

$$
\frac{\theta_{-}}{\theta_{+}} \approx \frac{E_{+}}{E_{-}}
$$

This relation could be used to obtain an initial-state radiation enriched event sample. A better method is to employ a kinematic fit to test the hypothesis that the complete event consists of the observed electron and positron, together with a photon along the beam line. The constraint equations are:

$$
\begin{aligned}
2 E_{\text {beam }} & =E_{+}+E_{-}+E_{\gamma} \\
E_{\gamma} & =E_{+} \cos \theta_{+}+E_{-} \cos \theta_{-} \\
E_{+} \sin \theta_{+} & =E_{-} \sin \theta_{-}
\end{aligned}
$$



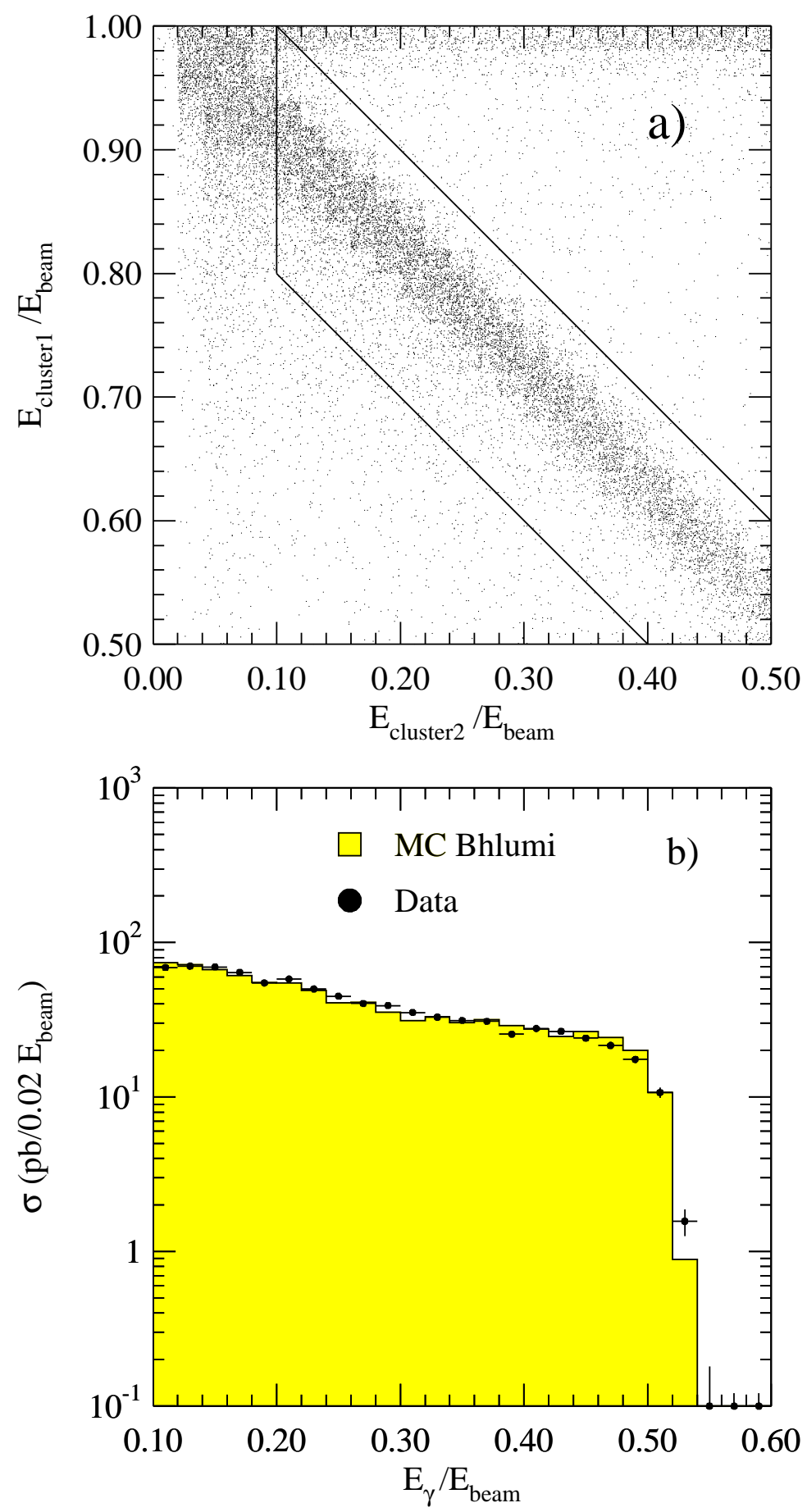

Figure 40: (a) The energy of the most and the least energetic clusters within one calorimeter, cluster 1 and 2 respectively, for final-state radiation candidates. The non-radiative events with an additional beam-gas interaction are clearly visible as the horizontal band at the top. The diagonal area indicates the energy requirements to select radiative events. (b) The differential cross section for final-state radiation as a function of the fractional photon energy. The points are the data and the histogram represents the BHLUMI Monte Carlo prediction. 
Based on these equations, the following $\chi^{2}$ is defined:

$$
\chi^{2}=\sum_{j=+-} \frac{\left(E_{j}-E_{j}^{\text {meas }}\right)^{2}}{\left(\Delta E_{j}\right)^{2}}+\frac{\left(\theta_{j}-\theta_{j}^{\text {meas }}\right)^{2}}{\left(\Delta \theta_{j}\right)^{2}} .
$$

The subscript $j$ indicates the electron $(j=-)$ or the positron $(j=+), \Delta E_{J}$ is the energy resolution and $\Delta \theta_{j}$ is the position resolution. The optimal photon energy, as well as improved electron (and positron) energies and polar scattering angles, are determined from a minimization of this $\chi^{2}$. Only events with a $\chi^{2}<5$ are kept to separate the initial-state radiation events from those Bhabha events with missing energy due to dead or malfunctioning crystals or reconstruction failures.

The measured differential cross section for initial-state photon radiation is given in Figure 41. In the same figure the result of the simulation is also indicated. The data and the simulation are in good agreement. To quantify the comparison, only events with a fitted photon energy exceeding $0.1 E_{\text {beam }}$ are kept. This sample amounts to about $4 \%$ of the total Bhabha event sample. The ratio of the integrated cross sections above $E_{\gamma}=0.10 E_{\text {beam }}$ is

$$
\frac{\int d \sigma / d E_{\gamma}(\text { Data }) d E_{\gamma}}{\int d \sigma / d E_{\gamma}(\mathrm{MC}) d E_{\gamma}}=(0.980 \pm 0.007 \pm 0.012),
$$

where the first uncertainty is again due to statistics, mostly from the Monte Carlo simulation, and the second uncertainty is due to the systematics. If the energy threshold in the data is shifted with respect to the Monte Carlo by the uncertainty in the energy scale of $0.1 \%$, the change in the above ratio is $1.2 \%$. This shows the need to accept the events in the radiative tail of the energy distribution. Due to the asymmetric energy cut, if the Bhabha energy selection criteria of $0.4 E_{\text {beam }}$ and $0.8 E_{\text {beam }}$ are shifted by $0.1 \%$ in the data with respect to the Monte Carlo, the change in integrated luminosity is less than $\mathbf{0 . 0 1 \%}$. Since no systematic difference between the data and the Monte Carlo is observed, no additional error is assigned to the luminosity determination apart from the theoretical error of $0.11 \%$ given in Section 5.2 . 


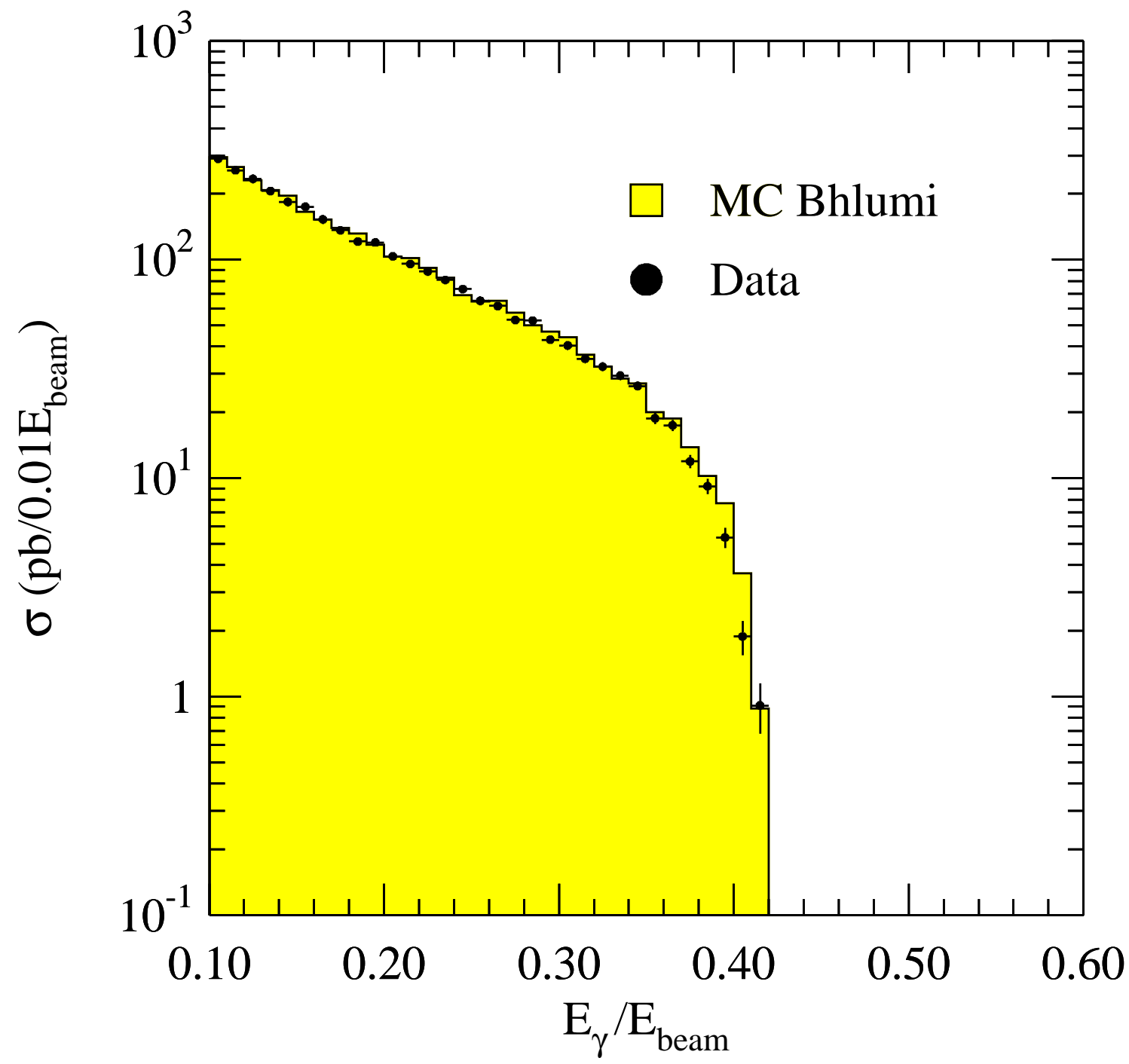

Figure 41: Differential cross section for initial-state radiation. The points are the data and the histogram represents the BHLUMI Monte Carlo prediction. 


\subsection{Systematic Error Summary}

The systematic errors on the luminosity determination are summarized in Table 5 . In this table, the errors for a luminosity measurement based on the BGO calorimeter only [16] are compared to those which include the silicon tracker. ${ }^{4)}$ The error due to event selection is estimated by

\begin{tabular}{|l|c|c|c|}
\hline \multirow{2}{*}{ Source } & \multicolumn{3}{|c|}{ Contribution to $\Delta \mathcal{L} / \mathcal{L}(\%)$} \\
\cline { 2 - 4 } & BGO Analysis & BGO+Silicon Analysis \\
\cline { 2 - 4 } & & 1993 & 1994 \\
\hline Trigger & Negligible & Negligible & Negligible \\
Event Selection & 0.3 & 0.04 & 0.05 \\
Background & Negligible & Negligible & Negligible \\
Geometry & 0.4 & 0.06 & 0.03 \\
\hline Total Experimental & 0.5 & 0.08 & 0.05 \\
\hline Monte Carlo Statistics & 0.06 & \multicolumn{3}{|c|}{0.06} \\
Theory & 0.11 & 0.11 \\
\hline Total & 0.5 & 0.15 & 0.14 \\
\hline
\end{tabular}

Table 5: Systematic uncertainties on the luminosity measurement.

varying each of the event selection cuts over a realistic domain and summing the contributions in quadrature. The results are shown in Figure 42 for the 1993 data and in Figure 43 for the 1994 data. The error bars shown in these plots indicate the error due to the change in statistics of each point with respect to a reference point (the nominal cut value) indicated by the arrow. The horizontal lines represent the systematic error assigned to each quantity. The total error assigned to event selection is $\mathbf{0 . 0 4 \%}$ in 1993 and $\mathbf{0 . 0 5 \%}$ in 1994 . The increased error due to the $\mathrm{E}_{\max }$ cut in 1994 is caused by events where the particle passes between the crystals and so deposits less energy. Such events mostly affect the distribution above the nominal cut of $0.8 E_{\text {beam. }}$. Below the cut the size of this effect can be seen to be small. A systematic error of $0.03 \%$ is assigned to this cut.

The uncertainties due to the detector geometry are summarized in Table 6 . In 1993, the main source of error was the knowledge of the distance in $z$ between the silicon detectors. In 1994, the knowledge of the wafer positions, temperature and $z$ distance each contributed about the same amount. The 1994 error of $0.026 \%$ is common to both years.

The total experimental systematic error is $\mathbf{0 . 0 8 \%}$ for 1993 and $\mathbf{0 . 0 5 \%}$ for 1994 . The total systematic error on the luminosity is $0.15 \%$ for 1993 and $0.14 \%$ for 1994 , where $0.14 \%$ of this error is fully correlated between the two years.

Since each of the four $r$-measuring layers of the silicon detector can be used to determine the luminosity, the individual measurements of the layers can be compared as a consistency check. The relative luminosities as determined by each of the 4 layers, $\mathcal{L}_{i} / \mathcal{L}_{w}$, are shown in Figure 44. The horizontal line in the figures is for an error of $0.06 \%$. This is derived from the event selection error, the wafer position error and the error due to the $z$ vertex of $0.3 \mathrm{~mm}$ (see Section 4.1.4). The measurements are seen to agree within the experimental systematic error.

\footnotetext{
4) The theoretical error for a measurement with the BGO calorimeter only is the same as for one with BGO + silicon. The error quoted in Reference [16] for the Monte Carlo generator, BHLUMI V2.01 has therefore been modified to reflect that of BHLUMI V4.03.
} 
1993
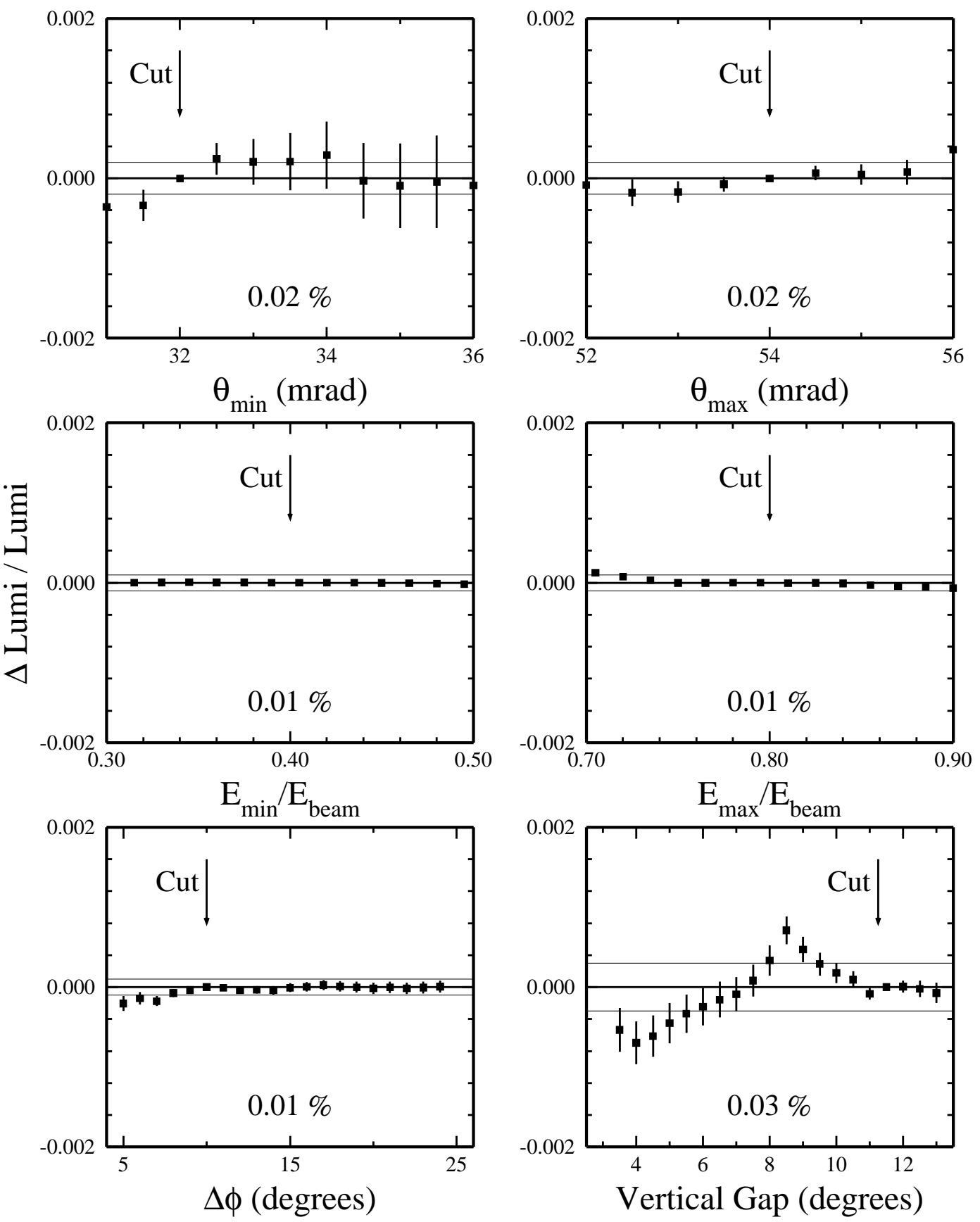

Figure 42: The fractional change in the measured luminosity as a function of the values of the different cut variables in 1993 . The standard cut value for each variable is indicated by an arrow. The systematic error assigned is shown by the horizontal lines in each plot. The variables are: (a) the tight minimum angle cut, (b) the tight maximum angle cut, (c) the minimum energy cut, $(d)$ the maximum energy cut, (e) the acoplanarity cut and (f) the tight vertical gap cut. 
1994
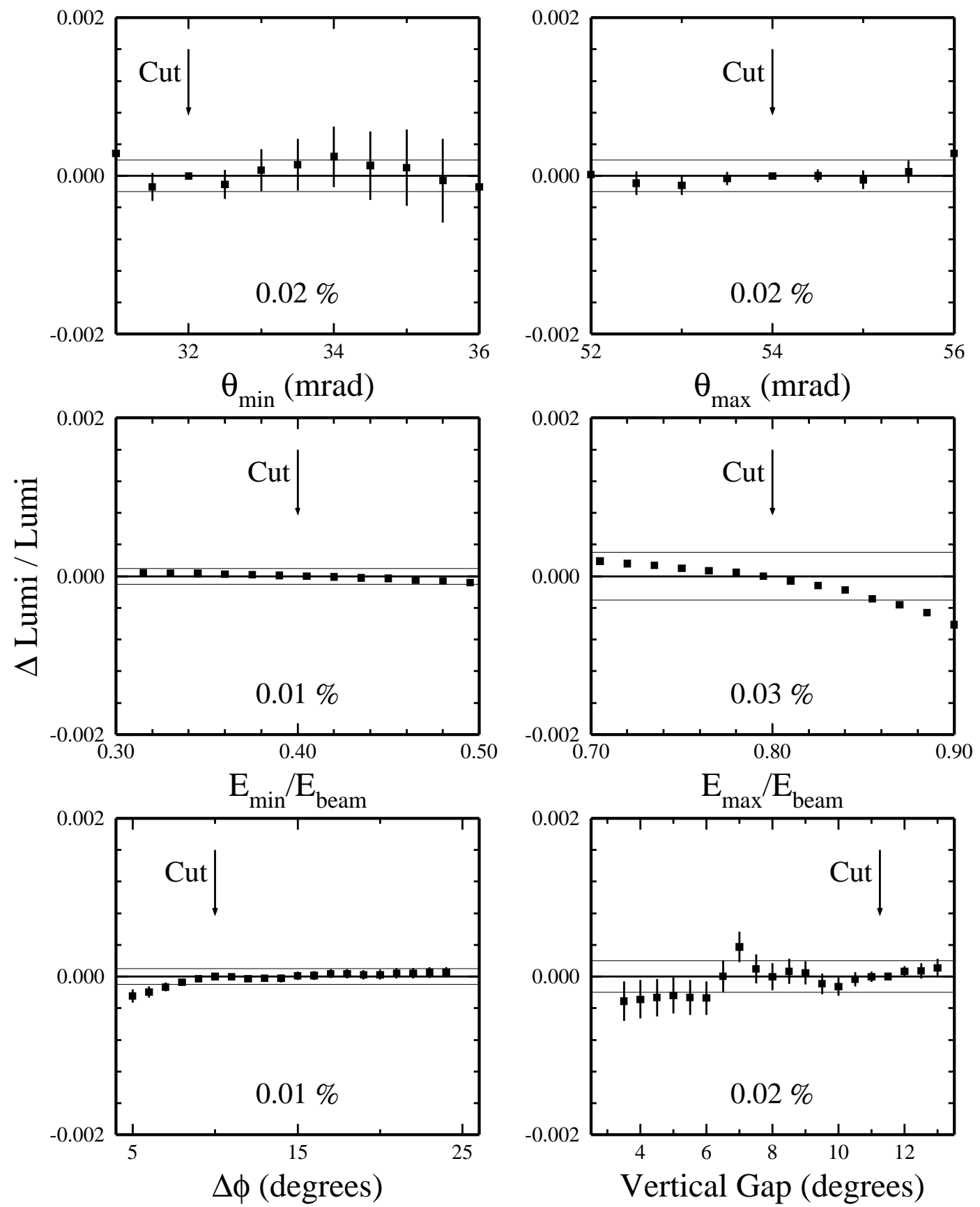

Figure 43: The fractional change in the measured luminosity as a function of the values of the different cut variables in 1994 . The standard cut value for each variable is indicated by an arrow. The systematic error assigned is shown by the horizontal lines in each plot. The variables are: (a) the tight minimum angle cut, (b) the tight maximum angle cut, (c) the minimum energy cut, $(d)$ the maximum energy cut, (e) the acoplanarity cut and (f) the tight vertical gap cut. 


\begin{tabular}{|l|l|c|l|c|}
\hline & \multicolumn{2}{|c|}{1993} & \multicolumn{2}{c|}{1994} \\
\cline { 2 - 5 } & Variation & Error & Variation & Error \\
\hline Wafer position & $\pm \mathbf{6 \mu \mathrm { m }}$ & $\mathbf{0 . 0 1 5 \%}$ & $\pm \mathbf{6 \mu \mathrm { m }}$ & $\mathbf{0 . 0 1 5 \%}$ \\
Temperature effects & $\pm 5^{\circ} \mathrm{C}$ & $\mathbf{0 . 0 1 4 \%}$ & $\pm 5^{\circ} \mathrm{C}$ & $\mathbf{0 . 0 1 4 \%}$ \\
$\boldsymbol{z}$ distance & $\pm 1.6 \mathrm{~mm}$ & $\mathbf{0 . 0 6 0 \%}$ & $\pm \mathbf{0 . 4} \mathrm{mm}$ & $\mathbf{0 . 0 1 6 \%}$ \\
\hline Total geometry & & $\mathbf{0 . 0 6 3 \%}$ & & $\mathbf{0 . 0 2 6 \%}$ \\
\hline
\end{tabular}

Table 6: The contributions of the uncertainty in the detector geometry to the systematic error.
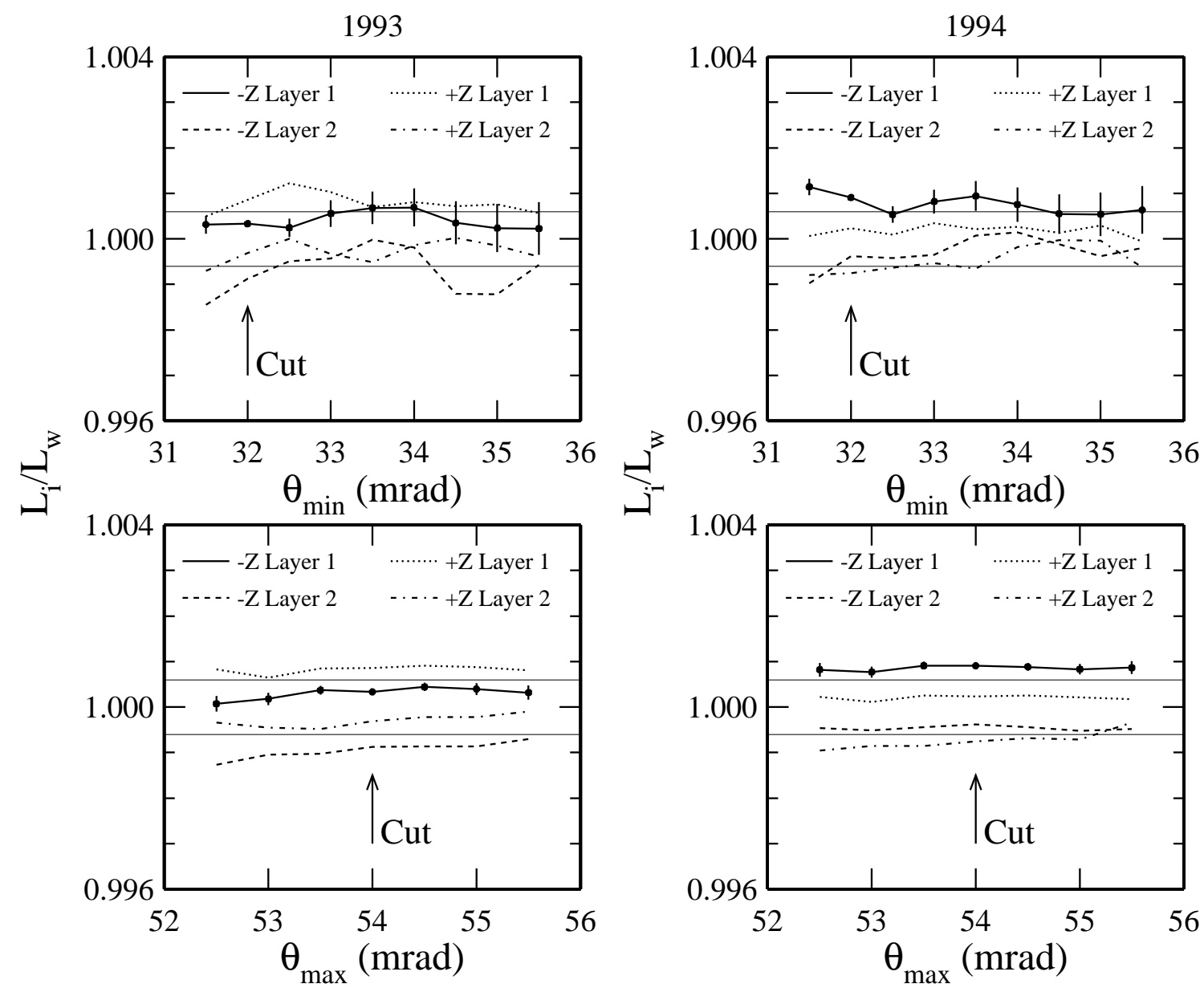

Figure 44: The ratio of the integrated luminosity in each of the $r$-layers to the final integrated luminosity, $\mathcal{L}_{w}$, as a function of the $\theta_{\text {min }}$ cut and $\theta_{\text {max }}$ cuts in 1993 and 1994. For clarity, the statistical error on only one of the ratios, due to the change in statistics relative to the cut value, is shown. The errors on the other ratios are the same. 


\section{Conclusions}

With a combination of a BGO calorimeter and a 3-layer silicon tracker in the L3 detector at LEP, the absolute luminosity has been measured with an experimental precision of $0.08 \%$ in 1993 and $0.05 \%$ in 1994 . Combining the experimental error with that due to limited Monte Carlo statistics $(\mathbf{0 . 0 6 \% )}$ ) and the uncertainty on the theoretical cross section $(0.11 \%)$ gives a total uncertainty of $0.15 \%$ in 1993 and $0.14 \%$ in 1994 . As a check a comparison between data and Monte Carlo for radiative Bhabha events shows excellent agreement.

\section{Acknowledgments}

The construction of the L3 luminosity monitor would not have been possible without the help of many engineers at the different collaborating institutes.

The work of J. Smith and J. Rudman from CMU was very important during the assembly of the BGO calorimeter. The support from members of the ECP division at CERN, particularly L. Veillet and P. Gervasoni, during the design and installation of the BGO calorimeter over many years is very much appreciated.

From NIKHEF we had invaluable support in the design and construction of the silicon tracker, especially from M. Gospic, W. Gotink, A. Rietmeyer and P. Zwikker. J. Rövekamp performed wonders with the bonding of the silicon wafers to the printed circuit boards. It is in no small part due to his efforts that we had so few dead channels.

E. Heijne and P. Jarron provided valuable information, as well as the AMPLEX chips. We acknowledge many fruitful discussions with Micron Semiconductor, particularly with C.D. Wilburn and A.D. Lucas.

The silicon tracker readout system was supplied by RWTH, Aachen. The provision of a working system with hardware and software considerably shortened the time between design and implementation of the silicon detector. We would like to thank C. Camps and H. Szczesny.

Financial support for the CMU group from the U.S. Department of Energy Contract No. DOE-ER-40682-115 is gratefully acknowledged.

\section{References}

[1] S.C.C. Ting et al., L3 Technical Proposal, Internal report, CERN, 1983;

L3 Collab., B. Adeva et al., Nucl. Inst. Meth. A 289 (1990) 35.

[2] S. Jadach et al., Phys. Lett. B 268 (1991) 253;

S. Jadach et al., Comp. Phys. Comm. 70 (1992) 305.

[3] S. Jadach et al., Phys. Lett. B 353 (1995) 362;

S. Jadach, W. Placzek and B.F.L. Ward, Phys. Lett. B 353 (1995) 349;

A. Arbuzov et al., "The present theoretical error on the Bhabha scattering cross section in the luminometry region at LEP", Preprint, 1996, Submitted to Phys. Lett. B.

[4] The L3 detector simulation is based on GEANT Version 3.15.

See R. Brun et al., "GEANT 3", CERN DD/EE/84-1 (Revised), September 1987.

[5] M. Goyot, B. Ille, P. Lebrun and J.P. Martin, Nucl. Inst. Meth. A 263 (1988) 180. 
[6] P. Kaaret, "Forward Production of $\mathrm{J} / \psi$ in Hadronic Interactions and Calibration of a Large BGO Electromagnetic Calorimeter", Ph.D. thesis, Princeton University, 1989, DOE/ER/3072-50.

[7] R. Bizzari et al., Nucl. Inst. Meth. A 317 (1992) 463;

P. Bagnaia et al., Nucl. Inst. Meth. A 324 (1993) 101;

F. Cesaroni et al., Nucl. Inst. Meth. A 323 (1992) 549;

P. Bagnaia et al., Nucl. Inst. Meth. A 323 (1992) 528.

[8] G.J. Bobbink et al., Nucl. Inst. Meth. 227 (1984) 470;

C. Laviron and P. Lecoq, Nucl. Inst. Meth. 227 (1984) 45.

[9] E. Beuville et al., in 5th European Symposium on Semiconductor Detectors, 21-23 February 1989, Munich, Germany, ed. A. Longoni et al., (Nucl. Inst. Meth., 1990), volume A 288, p. 157.

[10] F. Beissel et al., Nucl. Inst. Meth. A 332 (1993) 33.

[11] Micron Semiconductor Ltd., 1 Royal Buildings, Marlborough Road, Churchill Industrial Estate, Lancing, Sussex BN15 8UN, England.

[12] A.J. de Waard, "Hardware Description of the Carillon Module", Electronics Department, NIKHEF-H, Amsterdam, 1993, EH 819.13.13.

[13] The software was developed by the III. Physikalisches Institut, RWTH-Aachen. C. Camps, K. Hangarter: CM and DRP software, P. Schmitz: TAROT.

[14] M. Böhm, A. Denner and W. Hollik, Nucl. Phys. B 304 (1988) 687;

F.A. Berends, R. Kleis and W. Hollik, Nucl. Phys. B 304 (1988) 712.

[15] W. Beenakker and B. Pietrzyk, Phys. Lett. B 296 (1992) 241;

W. Beenakker and B. Pietrzyk, Phys. Lett. B 304 (1993) 366.

[16] L3 Collab., M. Acciarri et al., Z. Phys. C 62 (1994) 551. 